Supporting information for

\title{
Isarubrolones Containing a Pyridooxazinium Unit from Streptomyces as Autophagy Activators
}

Linli Li, ${ }^{\dagger}$ Shufen Li, ${ }^{\dagger}$ Bingya Jiang, ${ }^{,},+$Miaoqing Zhang, ${ }^{\dagger}$ Jingpu Zhang, ${ }^{\dagger}$ Beibei Yang, ${ }^{\ddagger}$ Li Li, Liyan Yu, ${ }^{\dagger}$ Hongyu Liu, ${ }^{\dagger}$ Xuefu You, ${ }^{\dagger}$ Xinxin $\mathrm{Hu},{ }^{\dagger}$ Zhen Wang, Yuhuan Li, ${ }^{\dagger}$ Linzhuan $\mathrm{Wu}^{*}{ }^{\dagger}$

${ }^{\dagger}$ NHC Key Laboratory of Biotechnology of Antibiotics, Key Laboratory of Synthetic Biology for Drug Innovation, Institute of Medicinal Biotechnology, Chinese Academy of Medical Sciences and Peking Union Medical College, Beijing 100050, People's Republic of China

${ }^{\ddagger}$ Institute of Materia Medica, Chinese Academy of Medical Sciences and Peking Union Medical College, Beijing 100050, People’s Republic of China 
Content

Item Title Page

The isarubrolone/isatropolone gene cluster from the genome DNA of Streptomyces sp. CPCC S6 204095 by AntiSMASH analysis.

$\begin{array}{ll}\text { Relative energy calculation } & \text { S37 }\end{array}$

$\begin{array}{ll}\text { ECD caculations. } & \text { S37 }\end{array}$

In vitro chemical reaction of isatropolone $\mathbf{C}$ (6) with glycine, aminoacetone or L-threonine.

Supplementing glycine in the medium increased the production of isarubrolone $\mathbf{E}$ (3) by S42 Streptomyces sp. CPCC 204095.

In vitro chemical reaction of isatropolone $\mathbf{C}(6)$ with some amino acids and amines.

A brief description of autopahgic activity assay.

A brief description of cytotoxicity assay.

Table S1. $\quad$ 16S rRNA gene sequence of Streptomyces sp. CPCC 204095 (for strain identification). S5

Figure S1. A rooted neighbour-joining distance tree of 16S rRNA gene of Streptomyces sp. CPCC S6 204095 with some Streptomyces strains.

Figure S2. Silica gel TLC of EtOAc extraction of Streptomyces sp. CPCC $204095 . \quad$ S6

Figure S3. HPLC analysis of EtOAc extract of Streptomyces sp. CPCC $204095 . \quad$ S7

Table S2. Annotation of the putative isarubrolone biosynthetic gene cluster from Streptomyces sp. S8 CPCC 204095

Figure S4. Organization of the putative isarubrolone/isatropolone gene cluster (isa) from S9 Streptomyces sp. CPCC 204095 as well as its comparison with isatropolone gene cluster (ist) from Streptomyces Gö66.

Figure S5. HPLC analysis of purple-red compound 1-4. S10

Figure S6. HPLC analysis of yellow compound 5-6. S11

Figure S7. HRESIMS analysis of isarubrolone C (1). $\quad$ S12

Figure S8. HRESIMS analysis of isarubrolone D (2). S12

Figure S9. HRESIMS analysis of isarubrolone E (3). $\quad$ S12

Figure S10. HRESIMS analysis of isarubrolone F (4). S13

Figure S11. HRESIMS analysis of isatropolone A (5). S13

Figure S12. HRESIMS analysis of isatropolone C (6). S13

Table S3. NMR data of isarubrolone C (1). S14

Table S4. NMR data of isarubrolone D (2). N15

Table S5. NMR data of isatroplone A (5). S16

$\begin{array}{ll}\text { Table S6. } & \text { NMR data of isatropolone C (6). }\end{array}$

$\begin{array}{llr}\text { Figure S13. } & { }^{1} \mathrm{H} \text { NMR spectrum of isarubrolone C (1). } & \text { S18 }\end{array}$

Figure S14. $\quad{ }^{13} \mathrm{C}$ NMR spectrum of isarubrolone C (1). $\quad S 18$

Figure S15. DEPT spectrum of isarubrolone C (1). $\quad$ S19

$\begin{array}{lll}\text { Figure S16. } & { }^{1} \mathrm{H}-{ }^{1} \mathrm{H} \text { COSY spectrum of isarubrolone } \mathrm{C}(\mathbf{1}) . & \text { S19 }\end{array}$

$\begin{array}{ll}\text { Figure S17. HSQC spectrum of isarubrolone C (1). } & \text { S20 }\end{array}$

$\begin{array}{lrr}\text { Figure S18. } & \text { HMBC spectrum of isarubrolone C (1). } & \text { S20 }\end{array}$

Figure S19. $\quad{ }^{1} \mathrm{H}$ NMR spectrum of isarubrolone D (2). $\quad$ S21

Figure S20. $\quad{ }^{13} \mathrm{C}$ NMR spectrum of isarubrolone D (2). S21 
Figure S21. DEPT spectrum of isarubrolone D (2).

$\begin{array}{lll}\text { Figure S22. } & { }^{1} \mathrm{H}-{ }^{1} \mathrm{H} \text { COSY spectrum of isarubrolone D (2). } & \text { S22 }\end{array}$

$\begin{array}{lll}\text { Figure S23. } & \text { HSQC spectrum of isarubrolone D (2). } & \text { S23 }\end{array}$

Figure S24. HMBC spectrum of isarubrolone D (2). $\quad$ S23

$\begin{array}{lll}\text { Figure S25. NOESY spectrum of isarubrolone D (2). } & \text { S24 }\end{array}$

$\begin{array}{lll}\text { Figure S26. } & { }^{1} \mathrm{H} \text { NMR spectrum of isarubrolone E (3). } & \text { S24 }\end{array}$

$\begin{array}{lll}\text { Figure S27. } & { }^{13} \mathrm{C} \text { NMR spectrum of isarubrolone E (3). } & \text { S25 }\end{array}$

$\begin{array}{lll}\text { Figure S28. } & \text { DEPT spectrum of isarubrolone E (3). } & \text { S25 }\end{array}$

$\begin{array}{lll}\text { Figure S29. } & { }^{1} \mathrm{H}-{ }^{1} \mathrm{H} \text { COSY spectrum of isarubrolone E (3). S26 }\end{array}$

$\begin{array}{lll}\text { Figure S30. HSQC spectrum of isarubrolone E (3). } & \text { S26 }\end{array}$

$\begin{array}{lll}\text { Figure S31. } & \text { HMBC spectrum of isarubrolone E (3). } & \text { S27 }\end{array}$

$\begin{array}{lll}\text { Figure S32. } & \text { NOESY spectrum of isarubrolone E (3). } & \text { S27 }\end{array}$

$\begin{array}{lll}\text { Figure S33. } & { }^{1} \mathrm{H} \text { NMR spectrum of isarubrolone F (4). } & \text { S28 }\end{array}$

$\begin{array}{lll}\text { Figure S34. } & { }^{13} \mathrm{C} \text { NMR spectrum of isarubrolone F (4). } & \text { S28 }\end{array}$

$\begin{array}{lrr}\text { Figure S35. } & \text { DEPT spectrum of isarubrolone F (4). S29 }\end{array}$

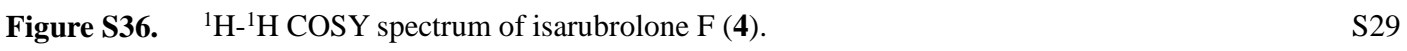

$\begin{array}{lll}\text { Figure S37. HSQC spectrum of isarubrolone F (4). S30 } & \text { S }\end{array}$

$\begin{array}{llr}\text { Figure S38. } & \text { HMBC spectrum of isarubrolone F (4). S30 }\end{array}$

$\begin{array}{ll}\text { Figure S39. NOESY spectrum of isarubrolone F (4). N31 } & \text { N }\end{array}$

$\begin{array}{lll}\text { Figure S40. } & { }^{1} \mathrm{H} \text { NMR spectrum of isatropolone A (5). } & \text { S31 }\end{array}$

$\begin{array}{lll}\text { Figure S41. } & { }^{13} \mathrm{C} \text { NMR spectrum of isatropolone A (5). S32 }\end{array}$

$\begin{array}{lll}\text { Figure S42. } & { }^{1} \mathrm{H}-{ }^{1} \mathrm{H} \text { COSY spectrum of isatropolone A (5). } & \text { S32 }\end{array}$

$\begin{array}{lll}\text { Figure S43. } & \text { HSQC spectrum of isatropolone A (5). } & \text { S33 }\end{array}$

Figure S44. HMBC spectrum of isatropolone A (5). S33

$\begin{array}{llc}\text { Figure S45. } & { }^{1} \mathrm{H} \text { NMR spectrum of isatropolone } \mathrm{C}(\mathbf{6}) . & \text { S34 }\end{array}$

$\begin{array}{lll}\text { Figure S46. } & { }^{13} \mathrm{C} \text { NMR spectrum of isatropolone } \mathrm{C}(\mathbf{6}) . & \text { S34 }\end{array}$

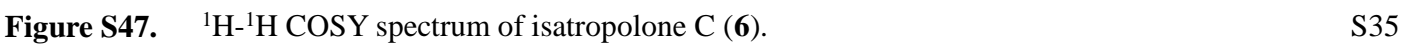

$\begin{array}{lll}\text { Figure S48. HSQC spectrum of isatropolone C (6). S35 } & \text { H }\end{array}$

$\begin{array}{lll}\text { Figure S49. } & \text { HMBC spectrum of isatropolone C }(\mathbf{6}) . & \text { S36 }\end{array}$

$\begin{array}{lll}\text { Figure S50. } & \text { Keto-enol tautomerization for isarubrolone C (1). } & \text { S37 }\end{array}$

Figure S51. (a) Structures of compound 1 (1Ba) and it isomers (1Aa, $1 \mathrm{Ab}$ and $1 \mathrm{Bb})$. (b) S38 Experimental CD spectrum with the spectra calculated for its configuration.

$\begin{array}{lll}\text { Figure S52. } & \text { Keto-enol tautomerization for isarubrolone D (2). S39 }\end{array}$

Figure S53. Assignment of absolute configuration of isarubrolone D (2) by comparing its S39 experimental CD spectrum with the spectra calculated for its configuration.

Figure S54. The plausible synthetic pathways for isarubrolone D (2) from isatropolone A (5) S40 conjugated with aminobenzoic acid or aminobenzamide (derived from chorismate).

Figure S55 LC-ESIMS of in vitro chemical reaction product of isatropolone C $(\mathbf{6}$, a) with glycine S41 (d), aminoacetone (e) and threonine (f).

Figure S56 HPLC comparison of isarubrolone E (3) produced by Streptomyces sp. CPCC 204095 S42 when glycine was supplemented into ISP2 medium.

Figure S57. The titer of isarubrolone F (4) in relation to L-threonine supplemented in the culture S43 medium for Streptomyces sp. CPCC 204095.

Figure S58. LC-ESIMS of in vitro chemical reaction product of isatropolone C $(\mathbf{6}$, a) with histidine $\quad$ S44 
(b), lysine (c) and 3-amino-2-methylbenzoic acid (d).

Figure S59. The autophagy inducing effect of isarubrolone C (1), isarubrolone E (3), isatropolone A S46 (5) and isatropolone $\mathrm{C}(\mathbf{6})$.

Table S60. IC $\mathrm{IC}_{50}$ of isarubrolone C (1) and isarubrolone F (4) on tumour cells. 
Table S1. 16S rRNA gene sequence of Streptomyces sp. CPCC 204095.

AGAGTTTGATCCTGGCTCAGGACGAACGCTGGCGGCGTGCTTAACACATGCAAGTCGA ACGATGAAGCCCTTCGGGGTGGATTAGTGGCGAACGGGTGAGTAACACGTGGGCAAT CTGCCCTTCACTCTGGGACAAGCCCTGGAAACGGGGTCTAATACCGGATAACACTCTG TCCCGCATGGGACGGGGTTGAAAGCTCCGGCGGTGAAGGATGAGCCCGCGGCCTATC AGCTTGTTGGTGGGGTGATGGCCTACCAAGGCGACGACGGGTAGCCGGCCTGAGAGG GCGACCGGCCACACTGGGACTGAGACACGGCCCAGACTCCTACGGGAGGCAGCAGTG GGGAATATTGCACAATGGGCGAAAGCCTGATGCAGCGACGCCGCGTGAGGGATGACG GCCTTCGGGTTGTAAACCTCTTTCAGCAGGGAAGAAGCGAGAGTGACGGTACCTGCA GAAGAAGCGCCGGCTAACTACGTGCCAGCAGCCGCGGTAATACGTAGGGCGCAAGCG TTGTCCGGAATTATTGGGCGTAAAGAGCTCGTAGGCGGCTTGTTGCGTCGGTTGTGAA AGCCCGGGGCTTAACCCCGGGTCTGCAGTCGATACGGGCAGGCTAGAGTGTGGTAGG GGAGATCGGAATTCCTGGTGTAGCGGTGAAATGCGCAGATATCAGGAGGAACACCGGT GGCGAAGGCGGATCTCTGGGCCATTACTGACGCTGAGGAGCGAAAGCGTGGGGAGCG AACAGGATTAGATACCCTGGTAGTCCACGCCGTAAACGTTGGGAACTAGGTGTTGGCG ACATTCCACGTCGTCGGTGCCGCAGCTAACGCATTAAGTTCCCCGCCTGGGGAGTACG GCCGCAAGGCTAAAACTCAAAGGAATTGACGGGGGCCCGCACAAGCAGCGGAGCATG TGGCTTAATTCGACGCAACGCGAAGAACCTTACCAAGGCTTGACATACACCGGAAAGC ATCAGAGATGGTGCCCCCCTTGTGGTCGGTGTACAGGTGGTGCATGGCTGTCGTCAGC TCGTGTCGTGAGATGTTGGGTTAAGTCCCGCAACGAGCGCAACCCTTGTTCTGTGTTG CCAGCATGCCCTTCGGGGTGATGGGGACTCACAGGAGACTGCCGGGGTCAACTCGGA GGAAGGTGGGGACGACGTCAAGTCATCATGCCCCTTATGTCTTGGGCTGCACACGTGC TACAATGGCCGGTACAATGAGCTGCGATGCCGCGAGGCGGAGCGAATCTCAAAAAGC CGGTCTCAGTTCGGATTGGGGTCTGCAACTCGACCCCATGAAGTCGGAGTTGCTAGTA ATCGCAGATCAGCATTGCTGCGGTGAATACGTTCCCGGGCCTTGTACACACCGCCCGTC ACGTCACGAAAGTCGGTAACACCCGAAGCCGGTGGCCCAACCCCTTGTGGGAGGGAG CTGTCGAAGGTGGGACTGGCGATTGGGACGAAGTCGTAACAAGGTAGCCGTACCGGA AGGTGCGGCTGGATCACCT

The mycelia from ISP2 medium plates were pooled for genomic DNA extraction following the manufacturer's instructions of DNeasy Blood \& Tissue kit (QIAGEN GROUP). The genomic DNA was sheared to ca. $10 \mathrm{~kb}$ for sequencing on the PacBio RSII with P4/C6 chemistry using the SMRTbell Template Prep Kit v1.0 according to the manufacturer's instructions. Sequence data were processed and assembled using HGAP3 with standard parameters. Sequence assembly was polished with the Quiver algorithm, as implemented in the PacBio SMRT analysis suite v2.3.0. The genomic DNA of Streptomyces sp. CPCC 204095 was assembled as one linear chromosome of $8,847,772 \mathrm{bp}$, one linear plasmid of $381,779 \mathrm{bp}$, from a total of 44,962,146 reads (including $8.99 \mathrm{~Gb}$ of DNA sequence).

The 16S rRNA gene sequence of Streptomyces sp. CPCC 204095 was extracted as in TableS1. The sequence showed $99-100 \%$ identity to $16 \mathrm{~S}$ rRNA genes from various Streptomyces strains. Thus, the strain belongs to Streptomyces. 


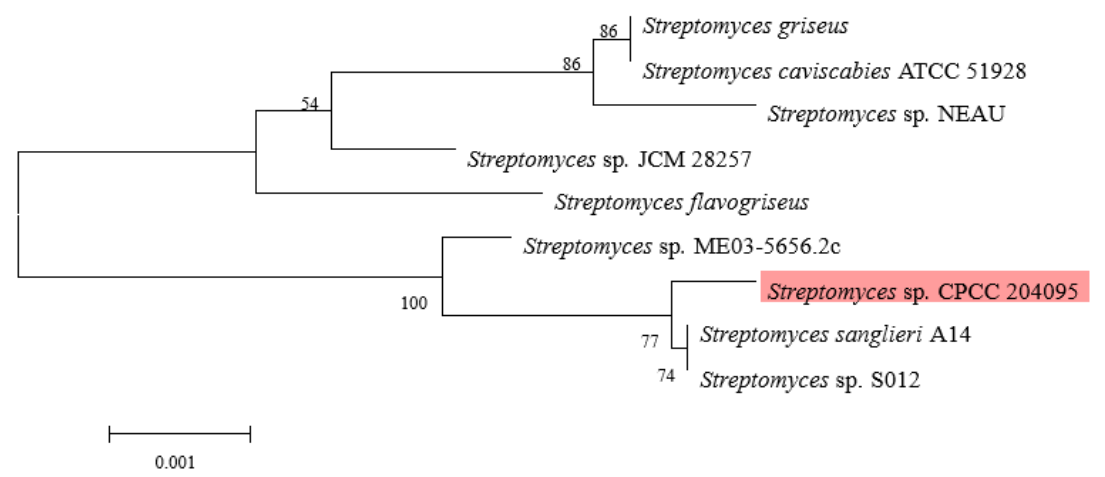

Figure S1. A rooted neighbour-joining distance tree of 16S rRNA gene of Streptomyces sp. CPCC 204095 with some Streptomyces strains.

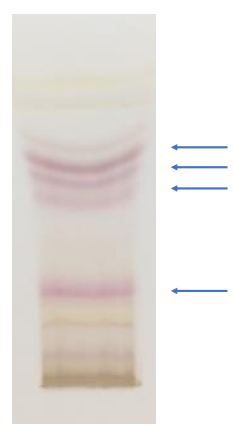

Figure S2. Silica gel TLC of EtOAc extraction of Streptomyces sp. CPCC 204095.

Four major purple red bands were indicated by arrows.

The isarubrolone/isatropolone gene cluster from the genome DNA of Streptomyces sp. CPCC 204095 by AntiSMASH analysis

The purple-red pigments produced by Streptomyces sp. CPCC 204095 might belong to aromatic polyketides from their UV-visible absorption profile. AntiSMASH analysis of the genomic DNA of Streptomyces sp. CPCC 204095 revealed only two gene clusters, Cluster 49 and Cluster 70, containing minimal PKS genes for aromatic polyketide biosynthesis in the genome of Streptomyces sp. CPCC 204095. Cluster 49 encoded for a T2pks_cf_fatty_acid, most similar to R1128, 42\% of genes show similarity. Cluster 70 encoded for a T2pks_terpene, most similar to spore pigment, $83 \%$ of genes show similarity.

There was a glycosyltransferase gene (isa 12) in Cluster 49. Its coding product showed $99 \%$ identity to IstK (glycosyltransferase) from the isarubrolone/isatropolone gene cluster of Streptomyces Gö66 by a further BLAST analysis in NCBI. Then, Cluster 49 was found to be nearly identical to the isarubrolone/isatropolone gene cluster from Streptomyces Gö66.

The purple-red pigments from Streptomyces sp. CPCC 204095 were found to have the same or similar UV-visible absorption profile with isarubrolone/isatropolone from Streptomyces Gö66. Thus, Cluster 49 should be the gene cluster for isarubrolone/isatropolone biosynthesis, and the purple-red pigments from Streptomyces sp. CPCC 204095 were deduced as isarubrolones. 


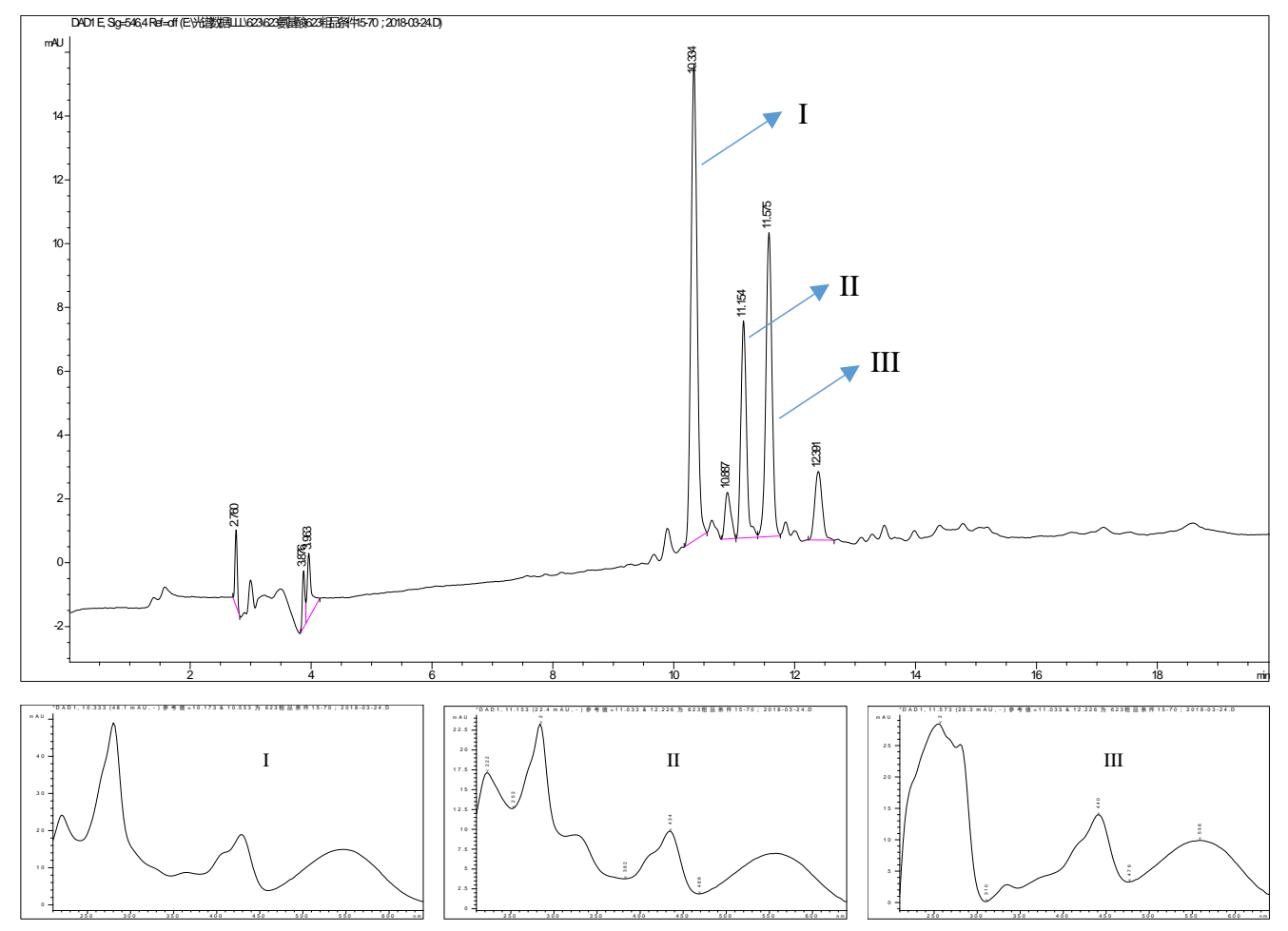

Figure S3. HPLC analysis of EtOAc extract of Streptomyces sp. CPCC 204095 incubated at $28{ }^{\circ} \mathrm{C}$ for 7 days. HPLC parameters: Diamonsil C18 $(4.6 \times 250 \mathrm{~mm}, 5 \mu)$; mobile phase $\mathrm{MeCN}-\mathrm{H}_{2} \mathrm{O}$ (containing $0.1 \% \mathrm{HAC}, v / v$ ) $, 1.0 \mathrm{~mL} / \mathrm{min}, 15-70 \%$ in $20 \mathrm{~min}$; wavelength $546 \mathrm{~nm} ; 27^{\circ} \mathrm{C}$.

Three peaks (I, II and III) with UV-visible absorption profile very similar to that of isarubrolone from Streptomyces Gö66 were detected. 
Table S2. Annotation of the putative isarubrolone/isatropolone biosynthetic gene cluster from Streptomyces sp. CPCC 204095

\begin{tabular}{|c|c|c|c|c|}
\hline Protein & Size & Putative Role & Identity/Positive & Accession No. \\
\hline Isa1 & 253 & Transcriptional activator [IstA Streptomyces Gö66] & $97 / 98$ & ARG41904.1 \\
\hline Isa2 & 204 & Regulator [IstB, Streptomyces Gö66] & $97 / 98$ & ARG41905.1 \\
\hline Isa3 & 281 & O-methyltransferase [IstC, Streptomyces Gö66] & $97 / 98$ & ARG41906.1 \\
\hline Isa4 & 493 & $\begin{array}{l}\text { drug resistance transporter [IstD, Streptomyces } \\
\text { Gö66] }\end{array}$ & $97 / 97$ & ARG41907.1 \\
\hline Isa5 & 526 & $\begin{array}{l}\text { Regulator FAD-like oxidase [IstE, Streptomyces } \\
\text { Gö66] }\end{array}$ & $99 / 99$ & ARG41908.1 \\
\hline Isa6 & 135 & $\begin{array}{l}\text { hypothetical protein RubN1 [Streptomyces sp. } \\
\text { KIB-H033] }\end{array}$ & $60 / 77$ & AOZ61193.1 \\
\hline Isa7 & 296 & SARP regulator [IstF, Streptomyces Gö66] & 98/98 & ARG41909.1 \\
\hline Isa8 & 84 & Acyl carrier protein [IstG, Streptomyces Gö66] & $94 / 98$ & ARG41910.1 \\
\hline Isa9 & 419 & Beta-ketosynthase [IstH, Streptomyces Gö66] & $99 / 100$ & ARG41911.1 \\
\hline Isa 10 & 436 & Chain length factor [IstI, Streptomyces Gö66] & $99 / 99$ & ARG41912.1 \\
\hline Isa11 & 263 & SARP family regulator [IstJ, Streptomyces Gö66] & 98/99 & ARG41913.1 \\
\hline Isa12 & 385 & Glycosyl transferase [IstK, Streptomyces Gö66] & $99 / 99$ & ARG41914.1 \\
\hline Isa13 & 370 & Dehydrogenase [IstL, Streptomyces Gö66] & $97 / 98$ & ARG41915.1 \\
\hline Isa14 & 276 & Cyclase/aromatase [IstM, Streptomyces Gö66] & $99 / 100$ & ARG41916.1 \\
\hline Isa15 & 249 & Cyclase [IstN, Streptomyces Gö66] & $99 / 100$ & ARG41917.1 \\
\hline Isa16 & 199 & Nitroreductase, MycH1 [IstO, Streptomyces Gö66] & $99 / 100$ & ARG41918.1 \\
\hline Isa17 & 597 & Oxygenase [IstP, Streptomyces Gö66] & $99 / 99$ & ARG41919.1 \\
\hline Isa18 & 136 & $\begin{array}{l}\text { Lipocalin-like domain-containing protein } \\
\text { [Streptomyces sp. Cf386] }\end{array}$ & $61 / 68$ & SDO52020.1 \\
\hline Isa19 & 280 & $\begin{array}{l}\text { NAD dependent epimerase/dehydratase, } \\
\text { SSOG_02370 [IstQ, Streptomyces Gö66] }\end{array}$ & 98/99 & ARG41920.1 \\
\hline Isa20 & 330 & $\begin{array}{l}\text { 3-oxoacyl-ACP synthase III [IstS, Streptomyces } \\
\text { Gö66] }\end{array}$ & $99 / 99$ & ARG41921.1 \\
\hline Isa21 & 409 & P450 monooxygenase [IstS, Streptomyces Gö66] & $99 / 99$ & ARG41922.1 \\
\hline Isa22 & 308 & Quinoe reductase [IstT, Streptomyces Gö66] & $99 / 99$ & ARG41923.1 \\
\hline Isa23 & 357 & $\begin{array}{l}\text { Malonyl-CoA acyltransferase [ IstU, Streptomyces } \\
\text { Gö66] }\end{array}$ & $97 / 98$ & ARG41924.1 \\
\hline Isa24 & 246 & $\begin{array}{l}\text { Microcin O-methyltransferase [Streptomyces } \\
\text { cyaneog- riseus] }\end{array}$ & $63 / 76$ & $\begin{array}{l}\text { WP_07896930 } \\
2.1\end{array}$ \\
\hline Isa25 & 390 & $\begin{array}{l}\text { Sugar O-methyltransferase [Streptomyces sp. } \\
\text { MK730-62F2] }\end{array}$ & $37 / 51$ & ACQ63636.1 \\
\hline Isa26 & 375 & $\begin{array}{l}\text { Isopentenyl-diphosphate } \\
\text { [Streptomyces sp. KIB-H033] }\end{array}$ & $63 / 74$ & AOZ61214.1 \\
\hline Isa27 & 283 & SARP regulator [IstF, Streptomyces Gö66] & $52 / 68$ & ARG41909.1 \\
\hline Isa28 & 364 & Aldo/keto reductase [Amycolatopsis sulphurea] & $61 / 71$ & $\begin{array}{l}\text { WP_09851354 } \\
8.1\end{array}$ \\
\hline Isa29 & 257 & $\begin{array}{l}\text { HxlR family transcriptional regulator [Streptomyces } \\
\text { olivochromogenes] }\end{array}$ & $84 / 88$ & $\begin{array}{l}\text { WP_06736022 } \\
0.1\end{array}$ \\
\hline
\end{tabular}




\begin{tabular}{|c|c|c|c|c|}
\hline Isa1' & 678 & $\begin{array}{l}\text { Glycosyltransferase family } 2 \text { protein [Streptomyces } \\
\text { atratus] }\end{array}$ & 93/95 & $\begin{array}{l}\text { WP_07248360 } \\
5.1\end{array}$ \\
\hline Isa2' & 199 & $\begin{array}{l}\text { dTDP-4-dehydrorhamnose } \\
\text { [Streptomyces } \text { sp. Ncost-T10-10d] }\end{array}$ & 95/97 & $\begin{array}{l}\text { WP_09389706 } \\
3.1\end{array}$ \\
\hline Isa3' & 361 & $\begin{array}{l}\text { Glucose-1-phosphate thymidylyltransferase } \\
\text { [Streptomyces atratus] }\end{array}$ & $98 / 98$ & SFX16924.1 \\
\hline Isa4' & 328 & $\begin{array}{l}\text { dTDP-glucose 4,6-dehydratase [Streptomyces sp. } \\
\text { DvalAA-43] }\end{array}$ & $99 / 99$ & $\begin{array}{l}\text { WP_09354259 } \\
8.1\end{array}$ \\
\hline Isa5' & 294 & $\begin{array}{l}\text { dTDP-4-dehydrorhamnose reductase [Streptomyces } \\
\text { atratus] }\end{array}$ & $97 / 98$ & $\begin{array}{l}\text { WP_07248360 } \\
8.1\end{array}$ \\
\hline Isa6' & 273 & Methyltransferase type 11 [Streptomyces atratus] & $95 / 98$ & $\begin{array}{l}\text { WP_07248360 } \\
7.1\end{array}$ \\
\hline Isa7' & 400 & $\begin{array}{l}\text { Glycosyltransferase family } 0 \text { protein [Streptomyces } \\
\text { atratus] }\end{array}$ & $94 / 97$ & SFX16946.1 \\
\hline
\end{tabular}

The GenBank accession number is MH588687 for the isarubrolone/isatropolone biosynthetic gene cluster (isa) from Streptomyces sp. CPCC 204095.

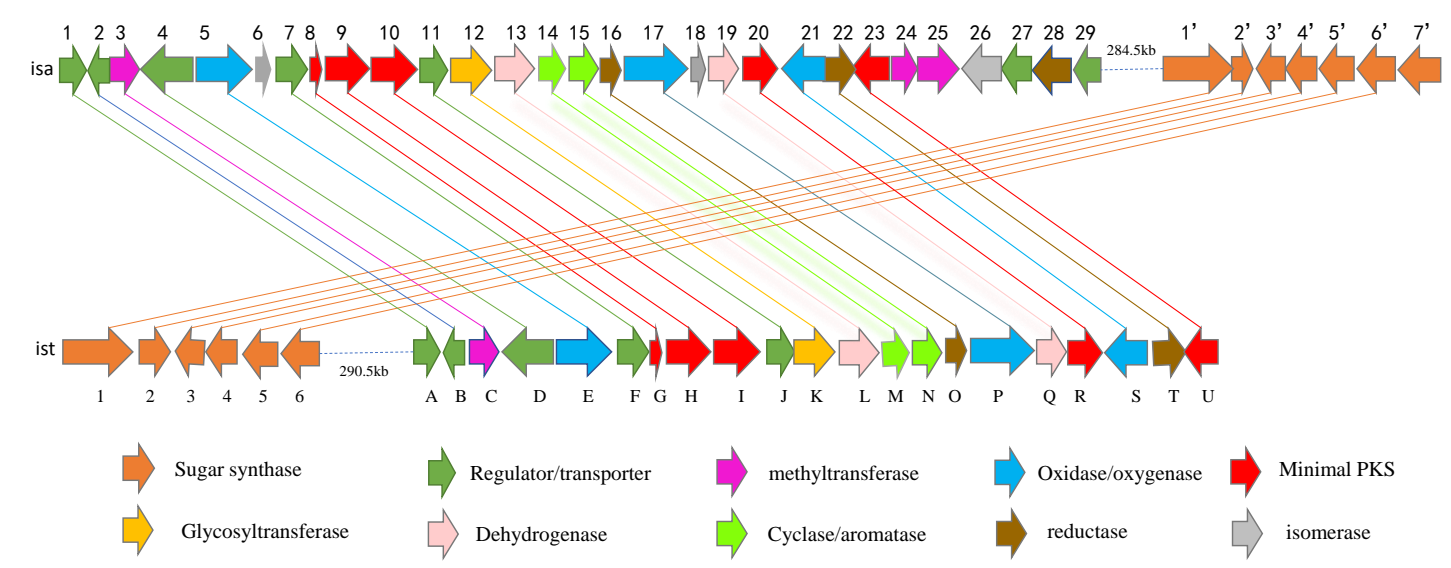

Figure S4. Organization of the putative isarubrolone/isatropolone gene cluster (isa) from Streptomyces sp. CPCC 204095 as well as its comparison with isatropolone gene cluster (ist) from Streptomyces Gö66. 


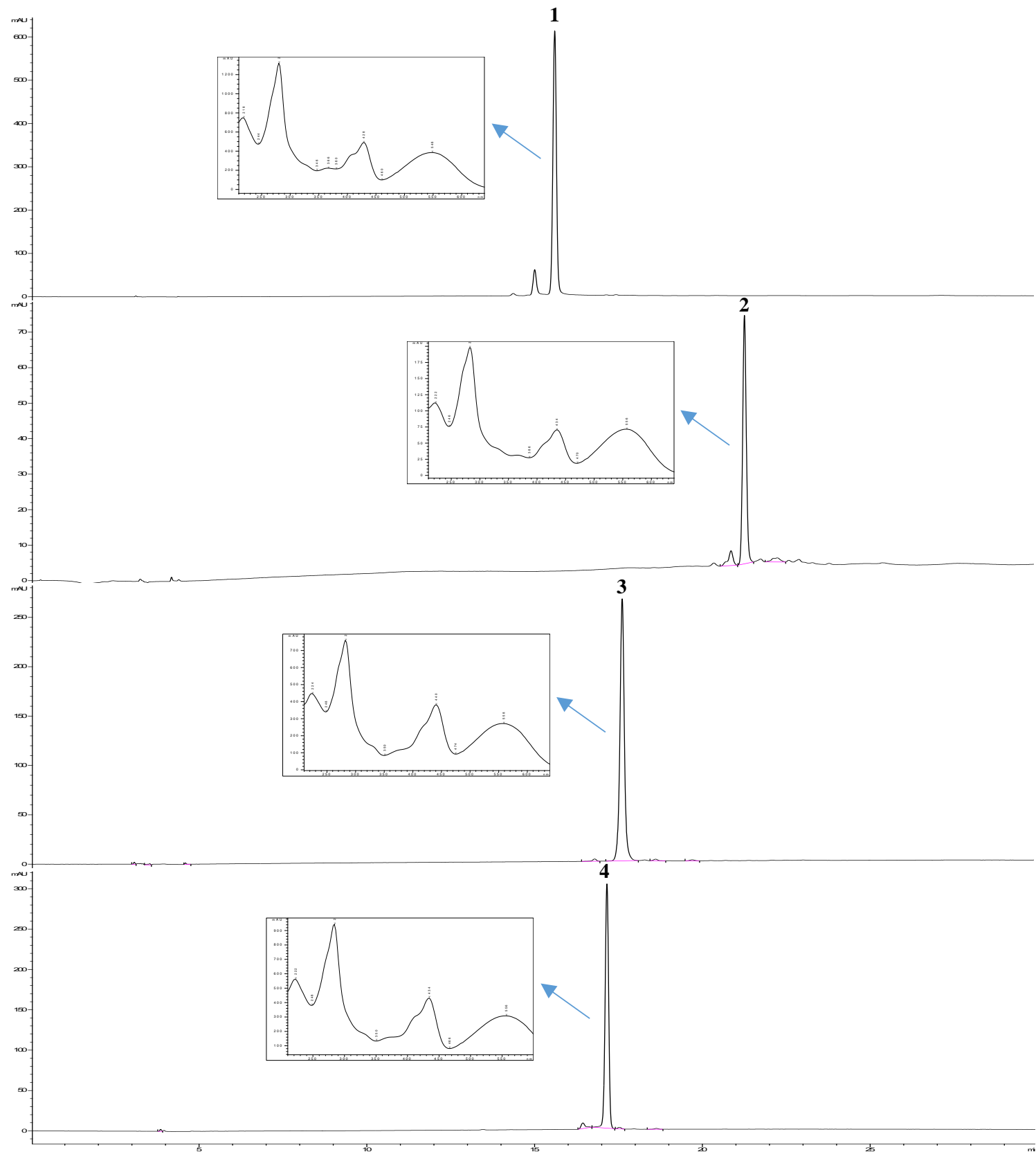

Figure S5. HPLC analysis of purple-red compound 1-4.

HPLC parameters: Diamonsil C18 $(4.6 \times 250 \mathrm{~mm}, 5 \mu)$; mobile phase $\mathrm{MeCN}-\mathrm{H}_{2} \mathrm{O}$ (containing $0.1 \% \mathrm{HAC}, v / \mathrm{v}), 1.0 \mathrm{~mL} / \mathrm{min}, 5-70 \%$ in $30 \mathrm{~min}$; wavelength $546 \mathrm{~nm} ; 27^{\circ} \mathrm{C}$. 


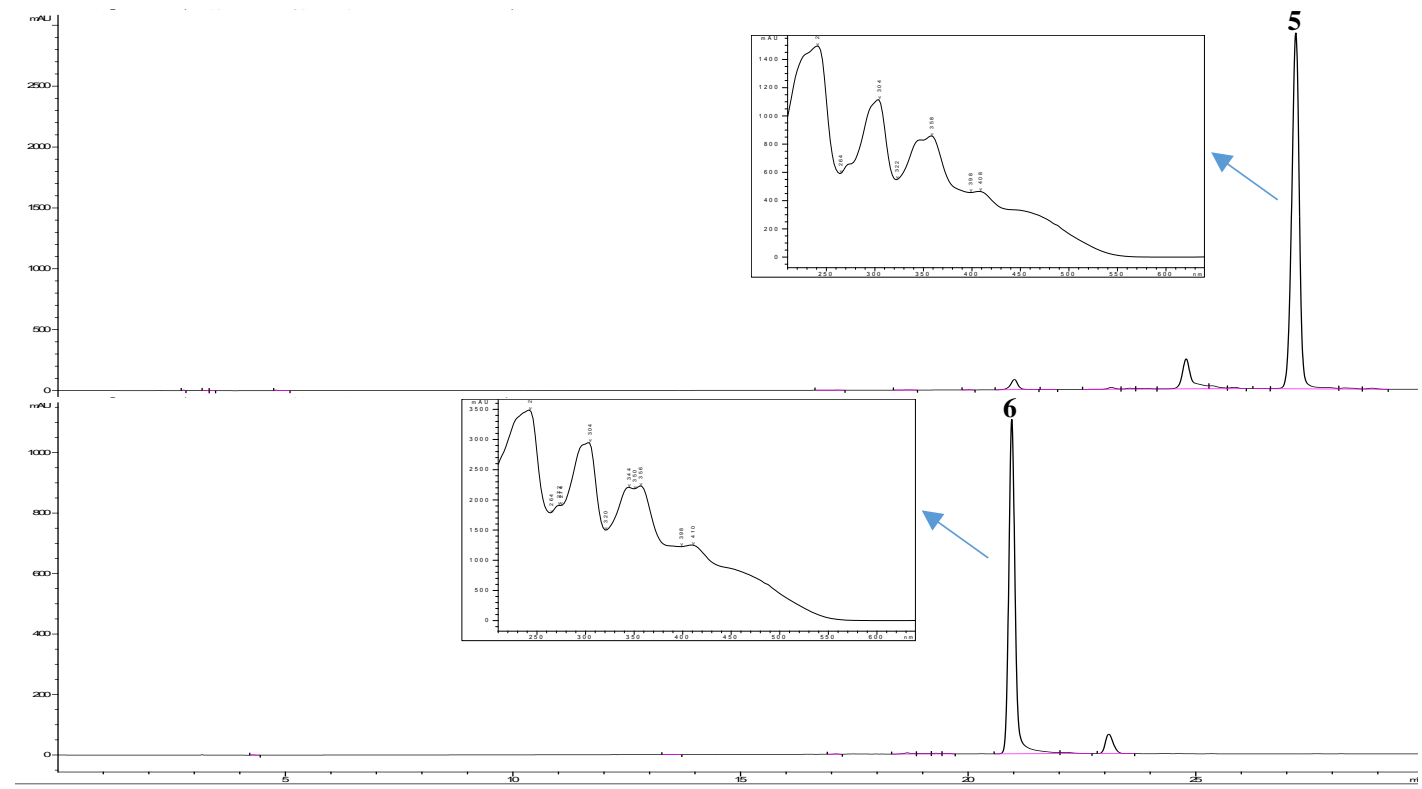

Figure S6. HPLC analysis of yellow compound 5-6.

HPLC parameters: Diamonsil C18 $(4.6 \times 250 \mathrm{~mm}, 5 \mu)$; mobile phase $\mathrm{MeCN}-\mathrm{H}_{2} \mathrm{O}$ (containing $0.1 \% \mathrm{HAC}, v / v), 1.0 \mathrm{~mL} / \mathrm{min}, 5-70 \%$ in $30 \mathrm{~min}$; wavelength $304 \mathrm{~nm} ; 27^{\circ} \mathrm{C}$. 


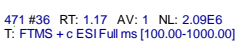

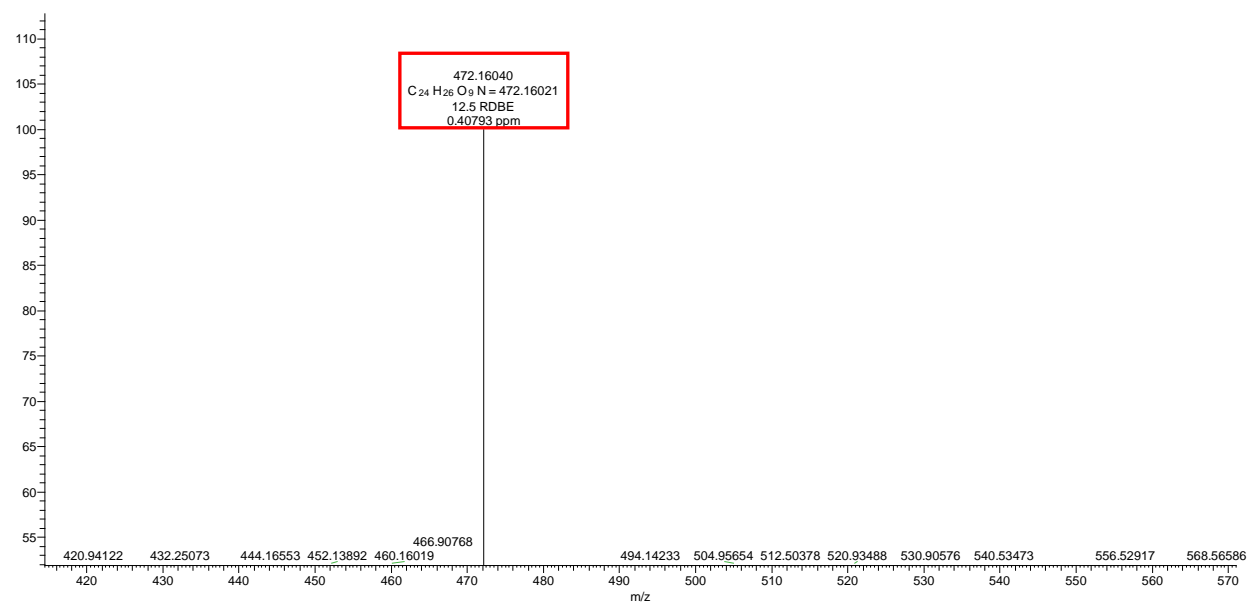

Figure S7. HRESIMS of isarubrolone C (1).

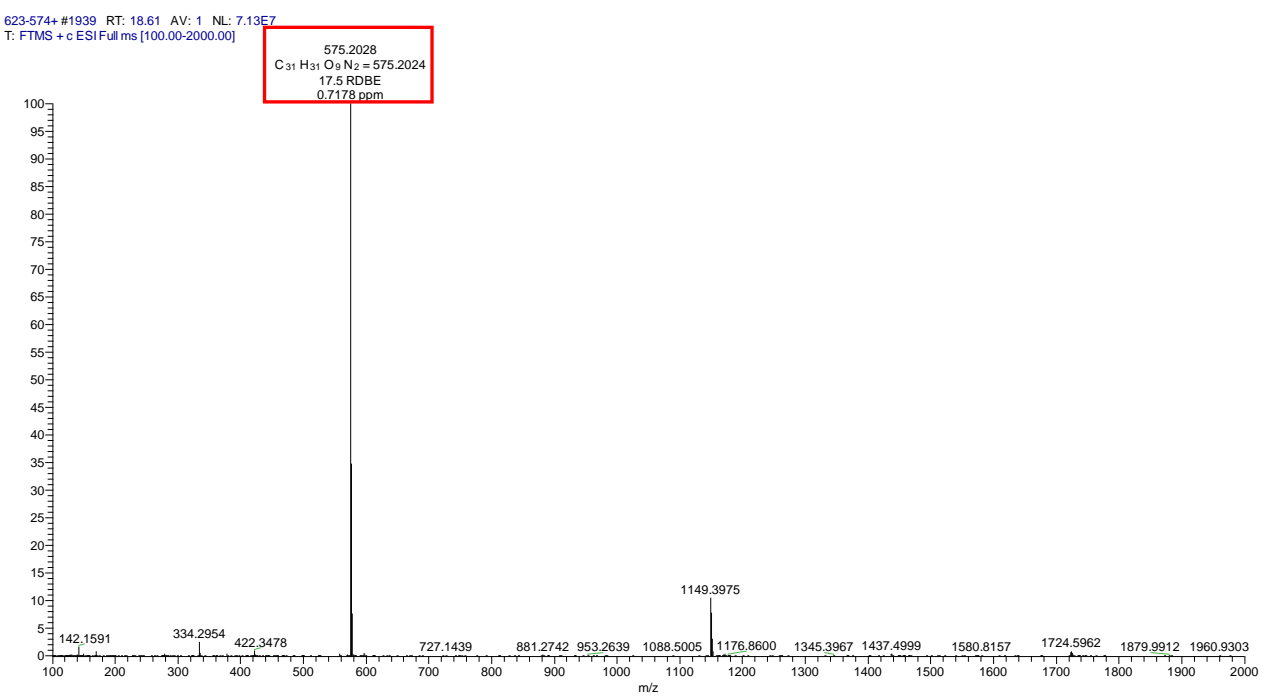

Figure S8. HRESIMS of isarubrolone D (2).

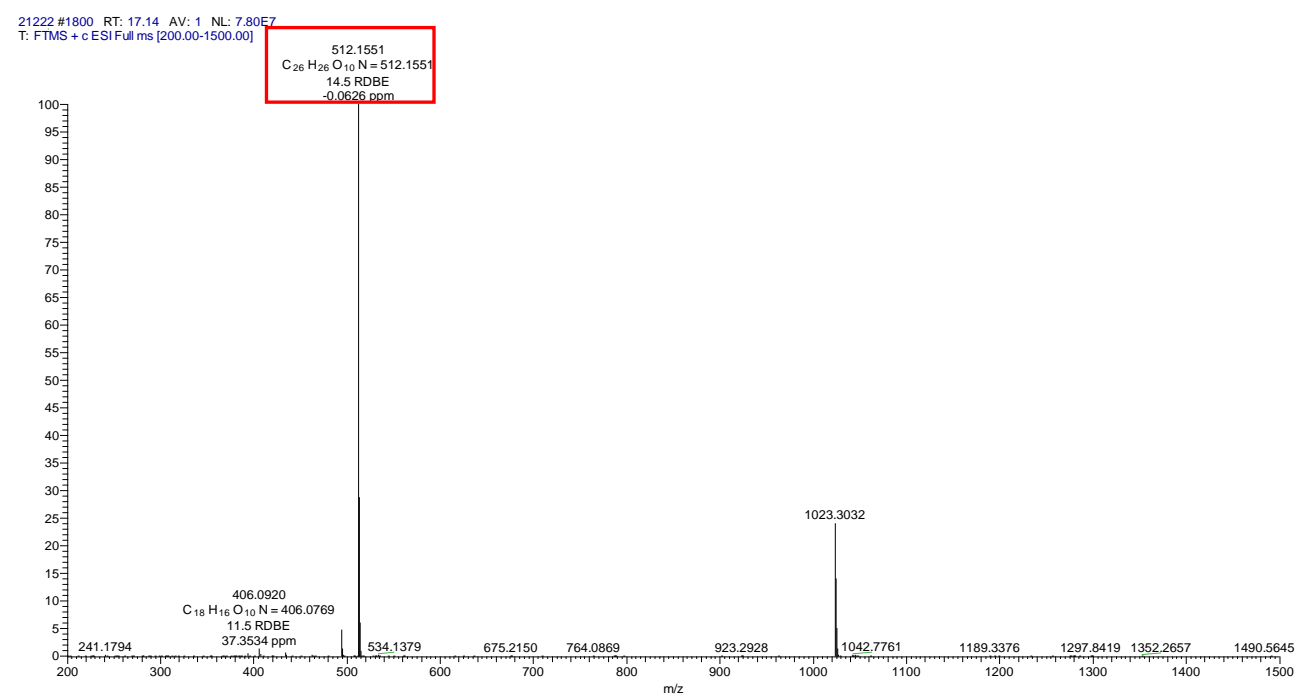

Figure S9. HRESIMS of isarubrolone E (3). 


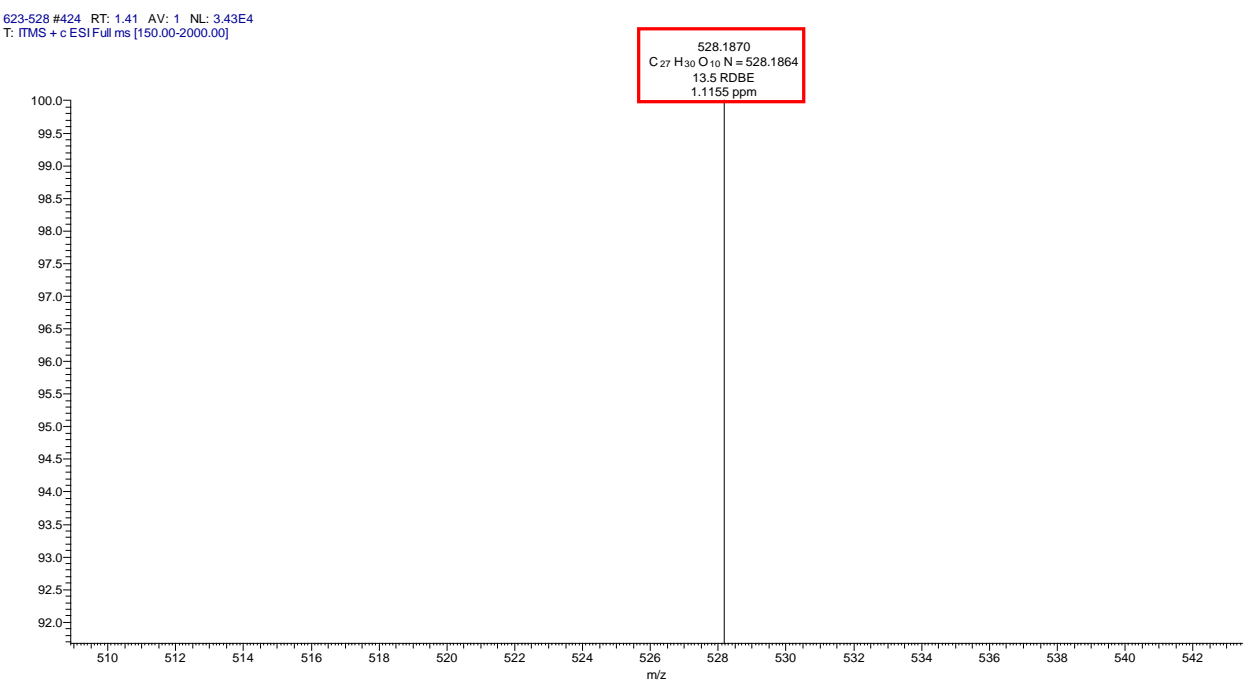

Figure S10. HRESIMS of isarubrolone F (4).

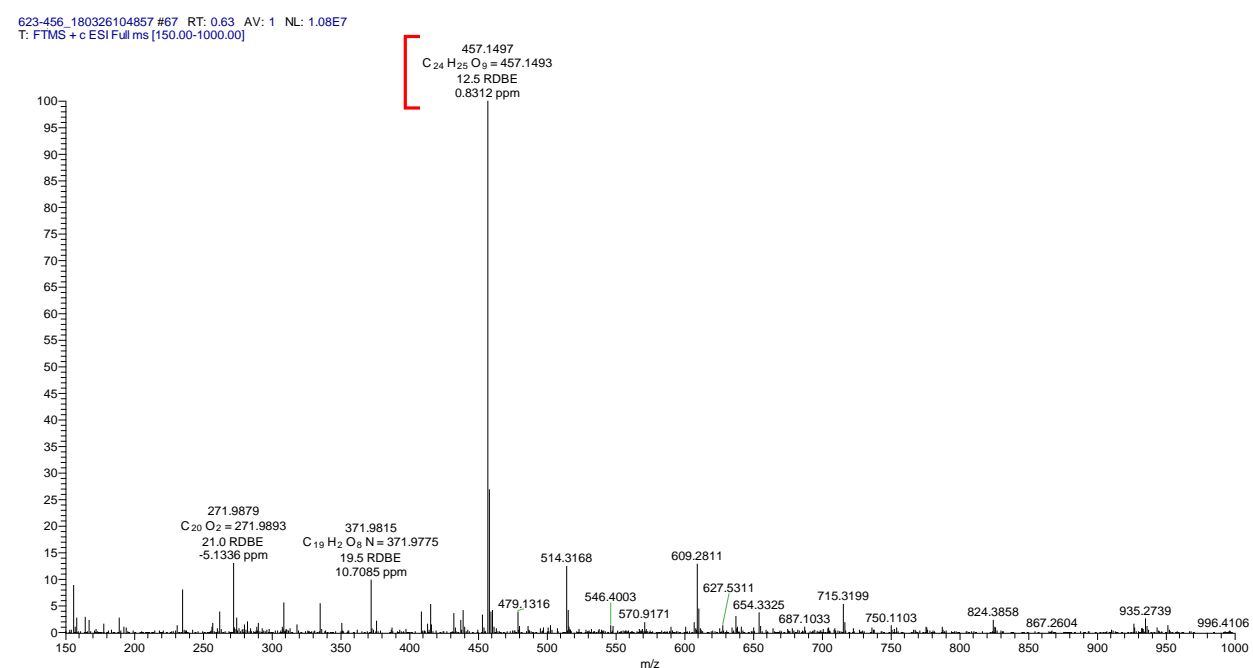

Figure S11. HRESIMS of isatropolone A (5).

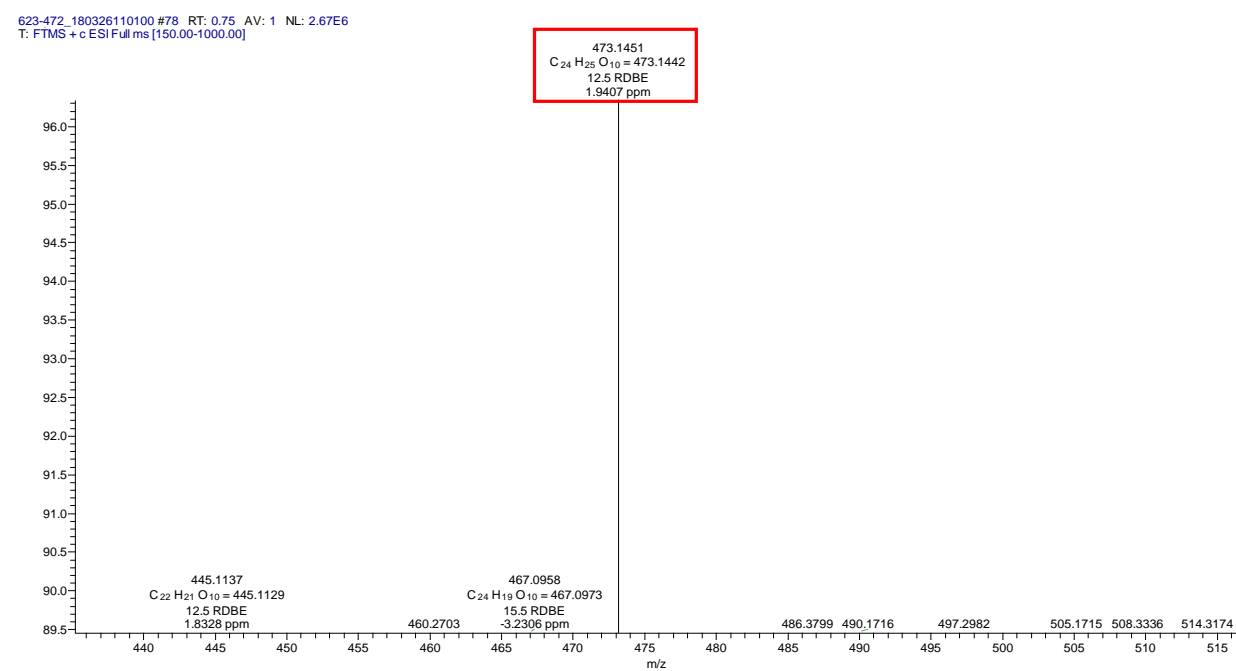

Figure S12. HRESIMS of isatropolone C (6). 
Table S3. NMR data of isarubrolone C (1) ( $\delta$ in ppm and $J$ in $\mathrm{Hz})$.

\begin{tabular}{|c|c|c|}
\hline position & $\delta_{\mathrm{C}}$, type & $\delta_{\mathrm{H}}, \quad$ mult, $(\mathrm{J}$ in $\mathrm{Hz})$ \\
\hline 1 & $10.3, \mathrm{CH}_{3}$ & $1.04, \mathrm{t}(7.2)$ \\
\hline 2 & $31.2, \mathrm{CH}_{3}$ & $1.76, \mathrm{~m}$ \\
\hline 3 & 69.6, $\mathrm{CH}$ & $5.43, \mathrm{~m}$ \\
\hline 4 & 157.7, C & \\
\hline 5 & $119.1, \mathrm{C}$ & \\
\hline 6 & 193.8, C & \\
\hline 7 & 116.7, CH & $6.61, \mathrm{~s}$ \\
\hline 8 & $185.5, \mathrm{C}$ & \\
\hline 9 & 128.6, C & \\
\hline 10 & 165.7, C & \\
\hline 11 & $170.2, \mathrm{C}$ & \\
\hline \multicolumn{3}{|l|}{$11-\mathrm{OH}$} \\
\hline 12 & $140.2, \mathrm{C}$ & \\
\hline 13 & 121.0, C & \\
\hline 14 & $159.3, \mathrm{C}$ & \\
\hline 15 & $118.7, \mathrm{CH}$ & $8.33, \mathrm{~s}$ \\
\hline 16 & 163.7, C & \\
\hline 17 & $21.4, \mathrm{CH}_{3}$ & $2.69, \mathrm{~s}$ \\
\hline $1^{\prime}$ & 109.1, CH & $5.66, \mathrm{~s}$ \\
\hline $2^{\prime}$ & 83.0, C & \\
\hline \multicolumn{3}{|l|}{ 2'-OH } \\
\hline $3^{\prime}$ & $70.8, \mathrm{CH}$ & 4.22 , brs \\
\hline \multicolumn{3}{|l|}{ 3'-OH } \\
\hline $4^{\prime}$ & $82.2, \mathrm{CH}$ & $3.32, \mathrm{~m}$ \\
\hline $5^{\prime}$ & $69.4, \mathrm{CH}$ & $3.94, \mathrm{~m}$ \\
\hline $6^{\prime}$ & $19.5, \mathrm{CH}_{3}$ & $1.29, \mathrm{~d}(6.6)$ \\
\hline $7^{\prime}$ & $58.7, \mathrm{CH}_{3}$ & $3.37, \mathrm{~s}$ \\
\hline
\end{tabular}


Table S4. NMR data of isarubrolone D (2) ( $\delta$ in ppm and $J$ in $\mathrm{Hz})$.

\begin{tabular}{|c|c|c|c|}
\hline \multicolumn{4}{|c|}{ isarubrolone D (2) } \\
\hline position & $\delta_{\mathrm{C}}$, type & $\delta_{\mathrm{H}}$, mult, $(J$ in $\mathrm{Hz})$ & HMBC \\
\hline 1 & $14.0, \mathrm{CH}_{3}$ & $0.73, \mathrm{t}(7.8)$ & $\mathrm{C}-2, \mathrm{C}-3$ \\
\hline 2 & 21.7, $\mathrm{CH}_{2}$ & $1.38, \mathrm{~m}$ & $\mathrm{C}-1, \mathrm{C}-4$ \\
\hline 3 & $30.5, \mathrm{CH}_{2}$ & $2.28, \mathrm{~m}$ & C-1. C-2, C-4, C-5 \\
\hline 4 & 154.6, C & & \\
\hline 5 & 120.6, C & & \\
\hline 6 & 192.9, C & & \\
\hline 7 & 117.1, CH & $6.42, \mathrm{~s}$ & C-6, C-8, C-9, C-12, C-13 \\
\hline 8 & 183.6, C & & \\
\hline 9 & 126.3, C & & \\
\hline 10 & 161.6, C & & \\
\hline 11 & 169.9, C & & \\
\hline \multicolumn{4}{|l|}{$11-\mathrm{OH}$} \\
\hline 12 & 138.0, C & & \\
\hline 13 & 111.3, C & & \\
\hline 14 & 162.1, C & & \\
\hline 15 & 115.6, C & 8.49. s & C-13, C-16, C-17 \\
\hline 16 & $158.2, \mathrm{C}$ & & \\
\hline 17 & $22.8, \mathrm{CH}_{3}$ & $2.18, \mathrm{~s}$ & $\mathrm{C}-15, \mathrm{C}-16$ \\
\hline 18 & $134.9, \mathrm{C}$ & & \\
\hline 19 & $128.8, \mathrm{CH}$ & $7.77, \operatorname{td}(7.2,1.8)$ & $\mathrm{C}-18, \mathrm{C}-20, \mathrm{C}-21$ \\
\hline $19-\mathrm{OH}$ & - & - & - \\
\hline 20 & $132.0, \mathrm{CH}$ & $7.80, \operatorname{td}(7.2,1.8)$ & $\mathrm{C}-18, \mathrm{C}-19, \mathrm{C}-21, \mathrm{C}-22$ \\
\hline 21 & $131.3, \mathrm{CH}$ & $7.82, \operatorname{td}(7.2,1.8)$ & C-20. C-22 \\
\hline 22 & $129.4, \mathrm{CH}$ & $7.90, \mathrm{dd}(7.2,1.8)$ & $\begin{array}{c}\mathrm{C}-18, \mathrm{C}-20, \mathrm{C}-21, \mathrm{C}-23 \\
\mathrm{C}-24\end{array}$ \\
\hline 23 & 132.2, C & & \\
\hline 24 & 166.7, C & & \\
\hline 24-NH2 & & $8.22, \mathrm{~s} ; 7.62, \mathrm{~s}$ & $\mathrm{C}-24$ \\
\hline $1^{\prime}$ & $106.5, \mathrm{CH}$ & $5.44, \mathrm{~s}$ & \\
\hline $2^{\prime}$ & $81.9, \mathrm{C}$ & & \\
\hline $2^{\prime}-\mathrm{OH}$ & & $5.57, \mathrm{~s}$ & C-1', C-2' \\
\hline $3^{\prime}$ & $67.5, \mathrm{CH}$ & $4.15, \mathrm{~m}$ & C-9, C-2', C-4' \\
\hline 3'-OH & & $5.77, \mathrm{~s}$ & $\mathrm{C}-1^{\prime}, \mathrm{C}-2^{\prime}, \mathrm{C}-3^{\prime}$ \\
\hline $4^{\prime}$ & $79.8, \mathrm{CH}$ & $3.20, \mathrm{dd}(8.4,3.0)$ & C-2', C-5', C-6', C-7' \\
\hline $5^{\prime}$ & $66.3, \mathrm{CH}$ & $3.92, \mathrm{~m}$ & C-4' \\
\hline $6^{\prime}$ & $18.3, \mathrm{CH}_{3}$ & $1.22, \mathrm{~d}(6.0)$ & C-4', C-5' \\
\hline $7^{\prime}$ & $56.8, \mathrm{CH}_{3}$ & $3.29, \mathrm{~s}$ & C-4 \\
\hline
\end{tabular}


Table S5. NMR data of isatroplone A (5) ( $\delta$ in ppm and $J$ in $\mathrm{Hz})$.

\begin{tabular}{|c|c|c|}
\hline position & $\delta_{\mathrm{C}}$, type & $\delta_{\mathrm{H}}$, mult, $(\mathrm{J}$ in $\mathrm{Hz})$ \\
\hline 1 & $13.8, \mathrm{CH}_{3}$ & $0.90, \mathrm{t}(7.2)$ \\
\hline 2 & 17.1, $\mathrm{CH}_{2}$ & $1.55, \mathrm{~m}$ \\
\hline 3 & $43.2, \mathrm{CH}_{2}$ & $2.85, \mathrm{t}(7.2)$ \\
\hline 4 & 196.2, C & \\
\hline 5 & $114.9, \mathrm{C}$ & \\
\hline 6 & 187.5, C & \\
\hline 7 & 133.2, CH & $7.01, \mathrm{~s}$ \\
\hline 8 & $182.0, \mathrm{C}$ & \\
\hline 9 & 131.5, C & \\
\hline 10 & $153.4, \mathrm{C}$ & \\
\hline 11 & 143.2, C & \\
\hline 12 & $133.8, \mathrm{C}$ & \\
\hline 13 & 121.97, C & \\
\hline 14 & 160.0, C & \\
\hline 15 & $106.2, \mathrm{CH}$ & $7.68, \mathrm{~d}(0.6)$ \\
\hline 16 & 168.7, C & \\
\hline 17 & $20.8, \mathrm{CH}_{3}$ & $2.53, \mathrm{~s}$ \\
\hline $1^{\prime}$ & $108.3, \mathrm{CH}$ & $5.62, \mathrm{~s}$ \\
\hline $2^{\prime}$ & $81.7, \mathrm{C}$ & \\
\hline \multicolumn{3}{|l|}{ 2'-OH } \\
\hline $3^{\prime}$ & $65.7, \mathrm{CH}$ & 4.33 , brs \\
\hline \multicolumn{3}{|l|}{ 3'-OH } \\
\hline $4^{6}$ & $78.3, \mathrm{CH}$ & $3.18, \mathrm{dd}(9.0,2.4)$ \\
\hline $5^{\prime}$ & $65.5, \mathrm{CH}$ & $4.10, \mathrm{~m}$ \\
\hline $6^{\prime}$ & 17.6, $\mathrm{CH}_{3}$ & $1.24, \mathrm{~d}(6.0)$ \\
\hline $7^{\prime}$ & $56.0, \mathrm{CH}_{3}$ & $3.31, \mathrm{~s}$ \\
\hline
\end{tabular}


Table S6. NMR data of isatropolone C (6) ( $\delta$ in ppm and $J$ in $\mathrm{Hz})$.

\begin{tabular}{|c|c|c|}
\hline position & $\delta_{\mathrm{C}}$, type & $\delta_{\mathrm{H}}$, mult, $(\mathrm{J}$ in $\mathrm{Hz})$ \\
\hline 1 & $10.0, \mathrm{CH}_{3}$ & $0.91, \mathrm{t}(7.2)$ \\
\hline $2 \mathrm{a}$ & $26.9, \mathrm{CH}_{2}$ & $1.69, \mathrm{~m}$ \\
\hline $2 b$ & 26.9, $\mathrm{CH}_{2}$ & $1.34, \mathrm{~m}$ \\
\hline 3 & $74.9, \mathrm{CH}$ & $4.84, \mathrm{dd}(7.8,3.6)$ \\
\hline \multicolumn{3}{|l|}{$3-\mathrm{OH}$} \\
\hline 4 & 197.6, C & \\
\hline 5 & 113.1, C & \\
\hline 6 & $187.2, \mathrm{C}$ & \\
\hline 7 & $133.5, \mathrm{CH}$ & $7.01, \mathrm{~s}$ \\
\hline 8 & $181.9, \mathrm{C}$ & \\
\hline 9 & 131.7, C & \\
\hline 10 & $153.4, \mathrm{C}$ & \\
\hline 11 & $143.4, \mathrm{C}$ & \\
\hline 12 & 133.7, C & \\
\hline 13 & $122.2, \mathrm{C}$ & \\
\hline 14 & 161.0, C & \\
\hline 15 & 106.5, CH & $7.70, \mathrm{~s}$ \\
\hline 16 & $169.3, \mathrm{C}$ & \\
\hline 17 & $20.9, \mathrm{CH}_{3}$ & 2.56, brs \\
\hline $1^{\prime}$ & $108.3, \mathrm{CH}$ & $5.62, \mathrm{~s}$ \\
\hline $2^{\prime}$ & $81.7, \mathrm{C}$ & \\
\hline \multicolumn{3}{|l|}{ 2'-OH } \\
\hline $3^{\prime}$ & $65.7, \mathrm{CH}$ & 4.33 , brs \\
\hline \multicolumn{3}{|l|}{ 3'-OH } \\
\hline $4^{\prime}$ & $78.4, \mathrm{CH}$ & $3.18, \mathrm{dd}(9.0,1.8)$ \\
\hline $5^{\prime}$ & $65.5, \mathrm{CH}$ & $4.09, \mathrm{~m}$ \\
\hline $6^{\prime}$ & 17.6, $\mathrm{CH}_{3}$ & $1.24, \mathrm{~d}(6.1)$ \\
\hline $7^{\prime}$ & $56.1, \mathrm{CH}_{3}$ & $3.31, \mathrm{~s}$ \\
\hline
\end{tabular}




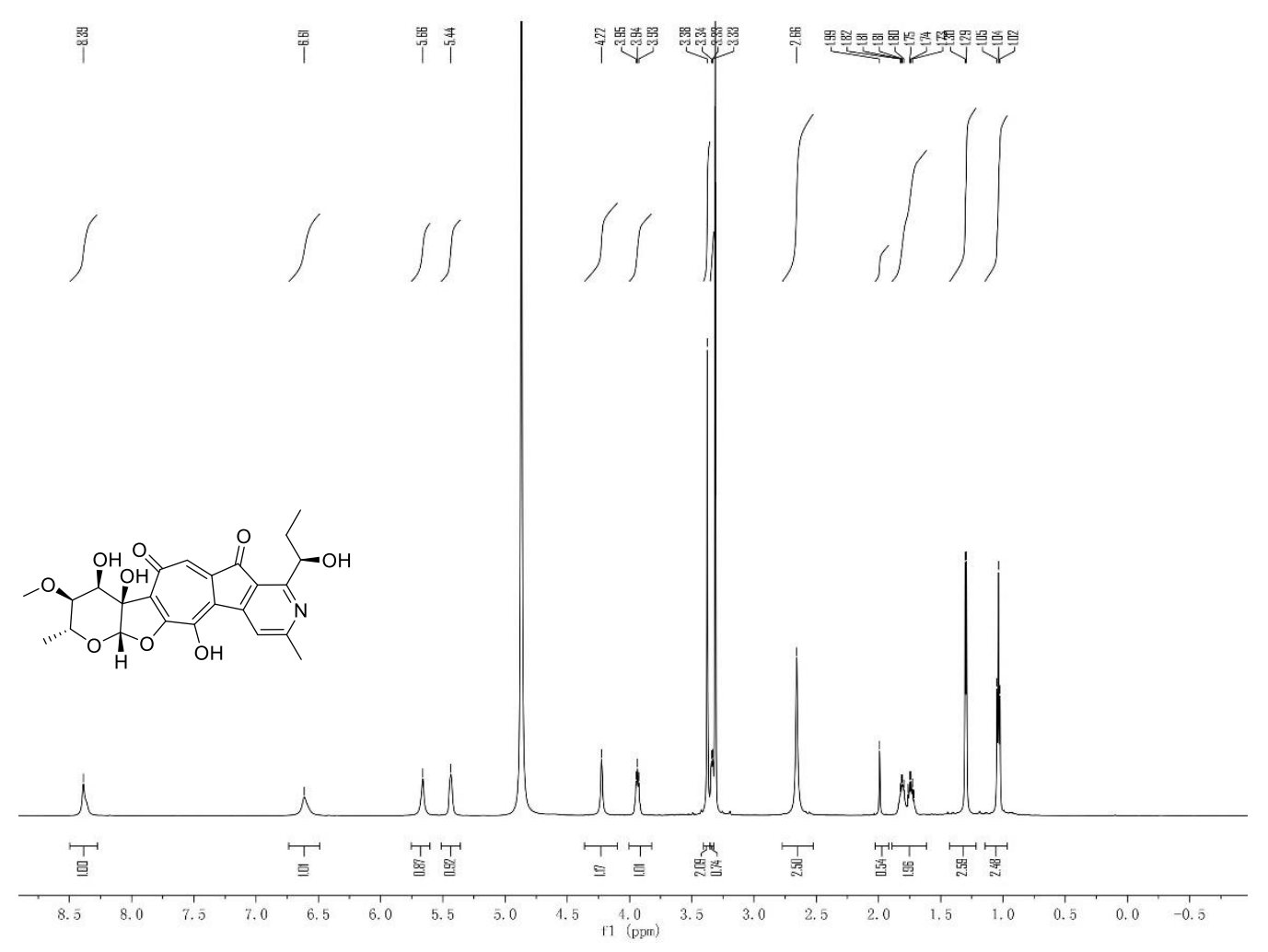

Figure S13. ${ }^{1} \mathrm{H}$ NMR spectrum of isarubrolone C (1).
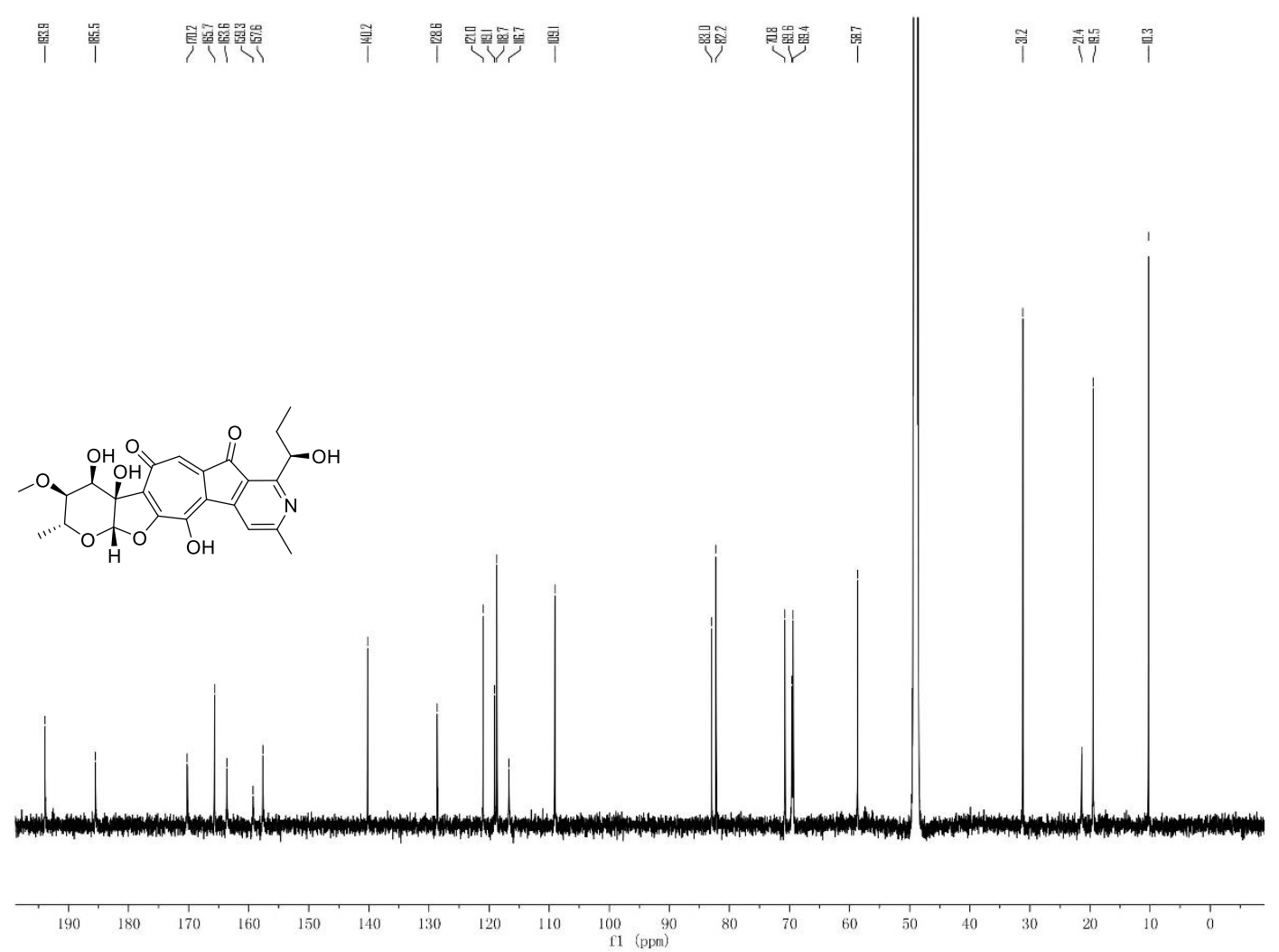

Figure S14. ${ }^{13} \mathrm{C}$ NMR spectrum of isarubrolone C (1). 

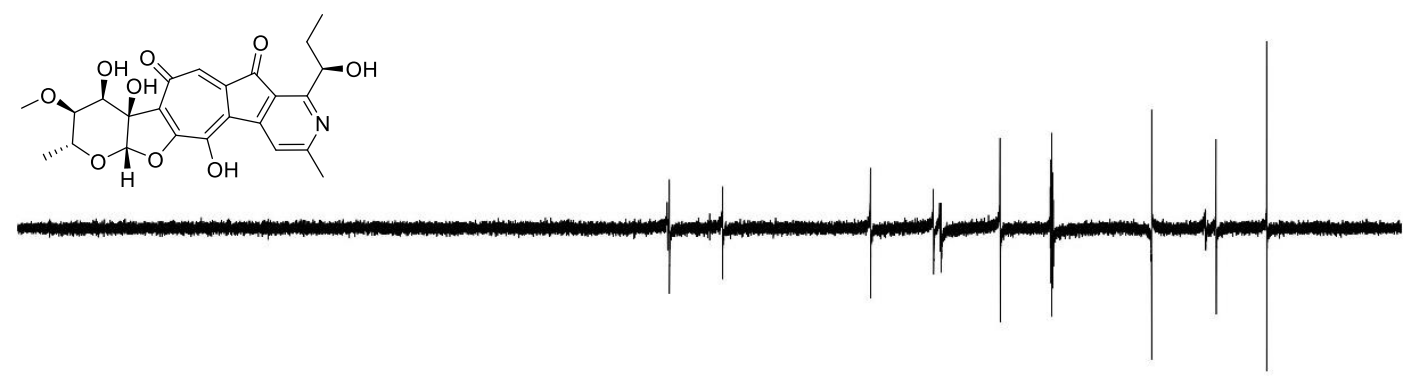

DIPPT 01

VNS-600 DEPT 623-36-42-01 IN ed3od Mar 292017

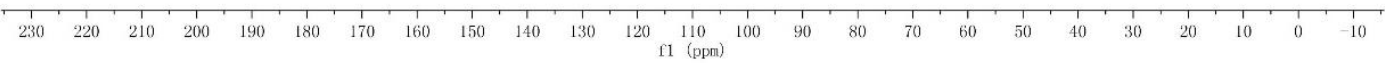

Figure S15. DEPT spectrum of isarubrolone C (1).

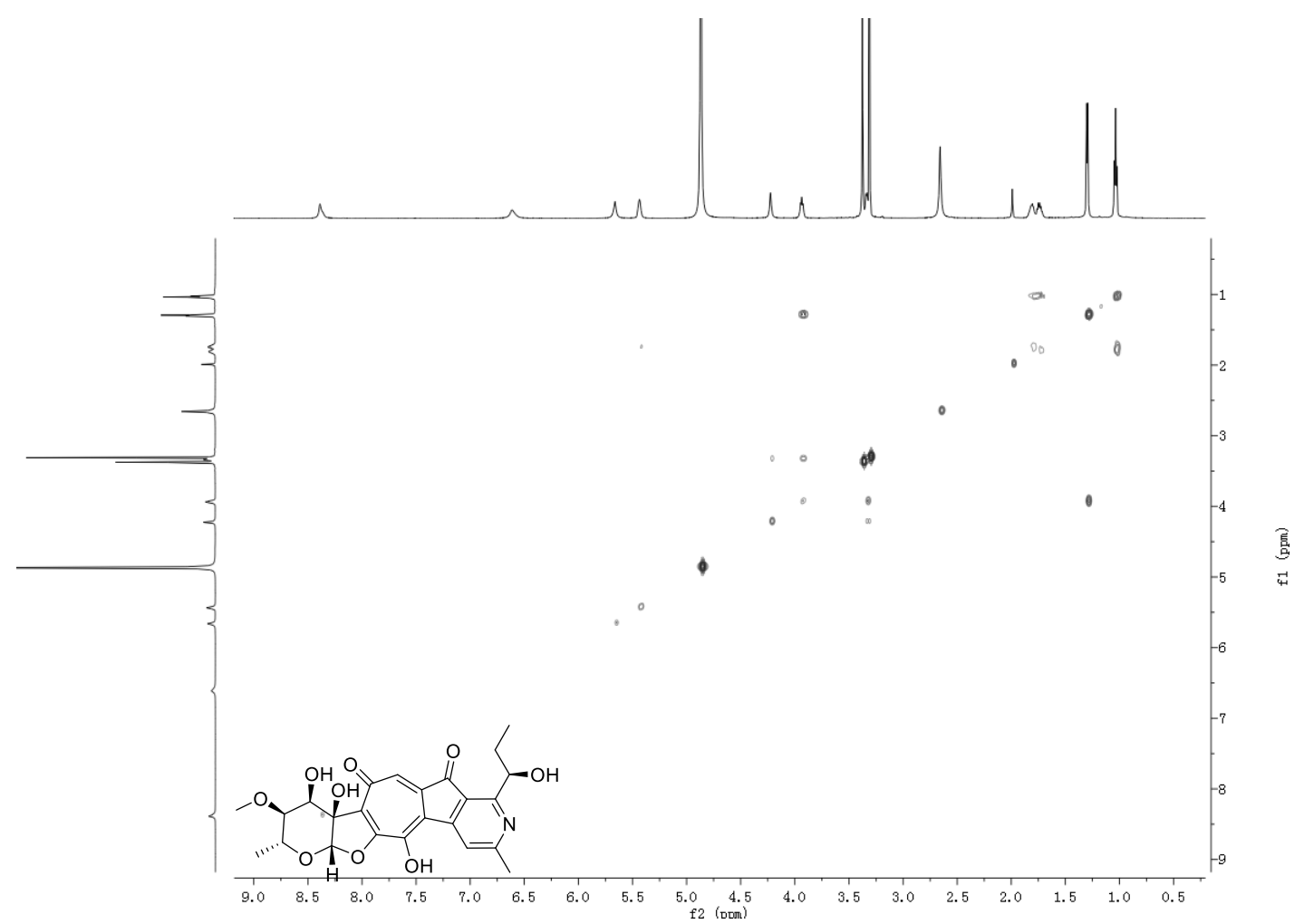

Figure S16. ${ }^{1} \mathrm{H}-{ }^{1} \mathrm{H}$ COSY spectrum of isarubrolone $\mathrm{C}(\mathbf{1})$. 


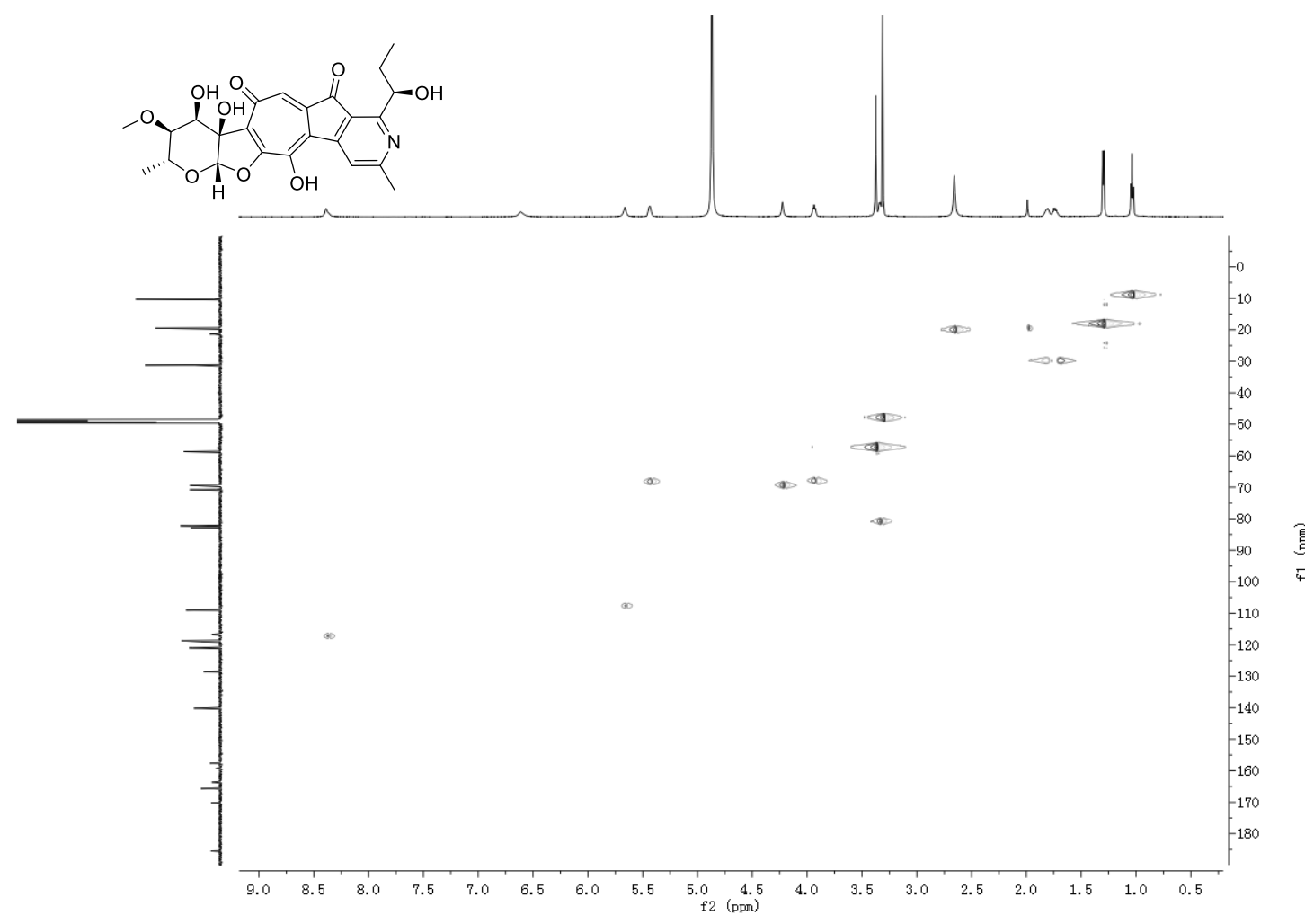

Figure S17. HSQC spectrum of isarubrolone C (1).

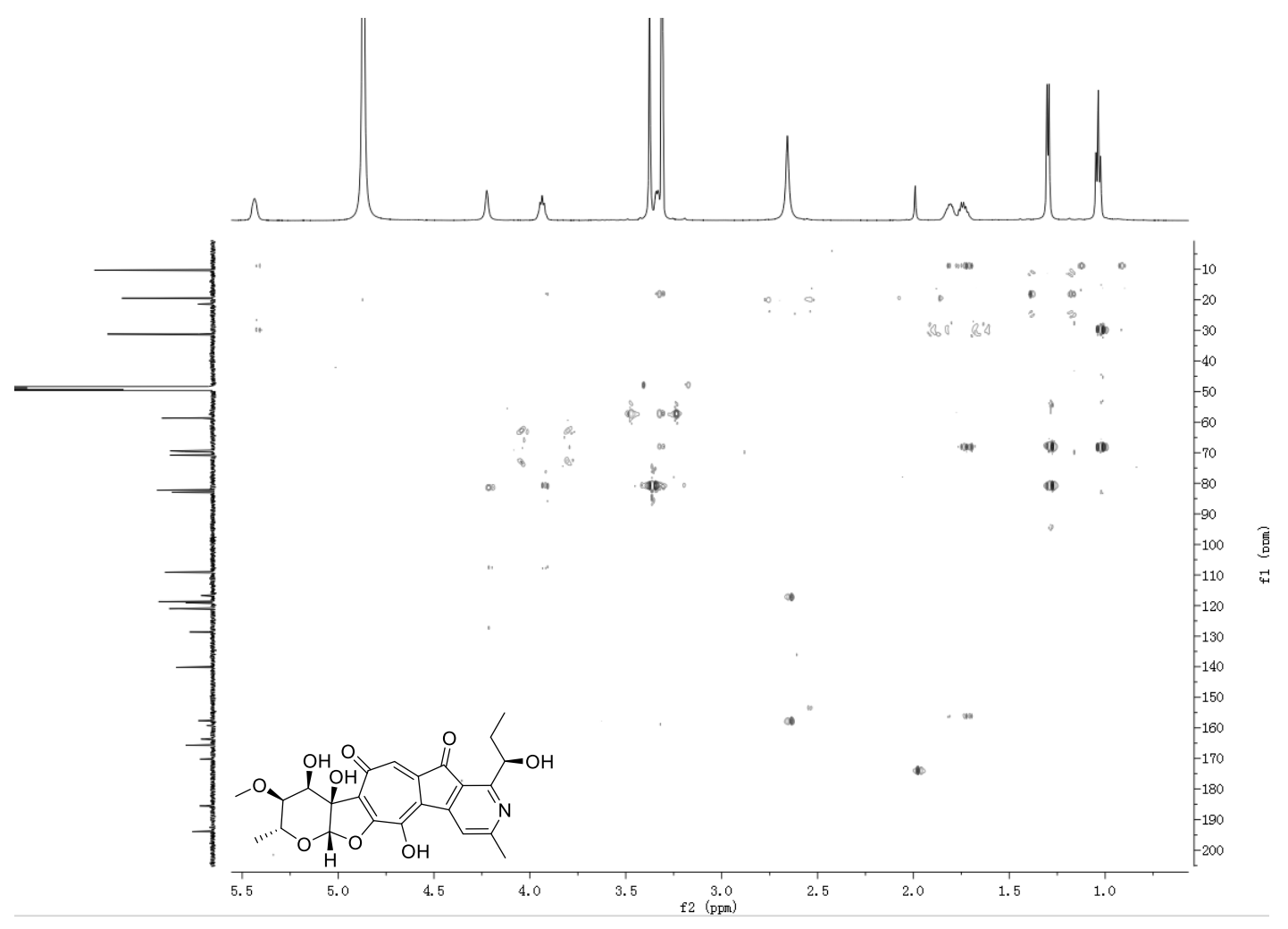

Figure S18. HMBC spectrum of isarubrolone C (1). 


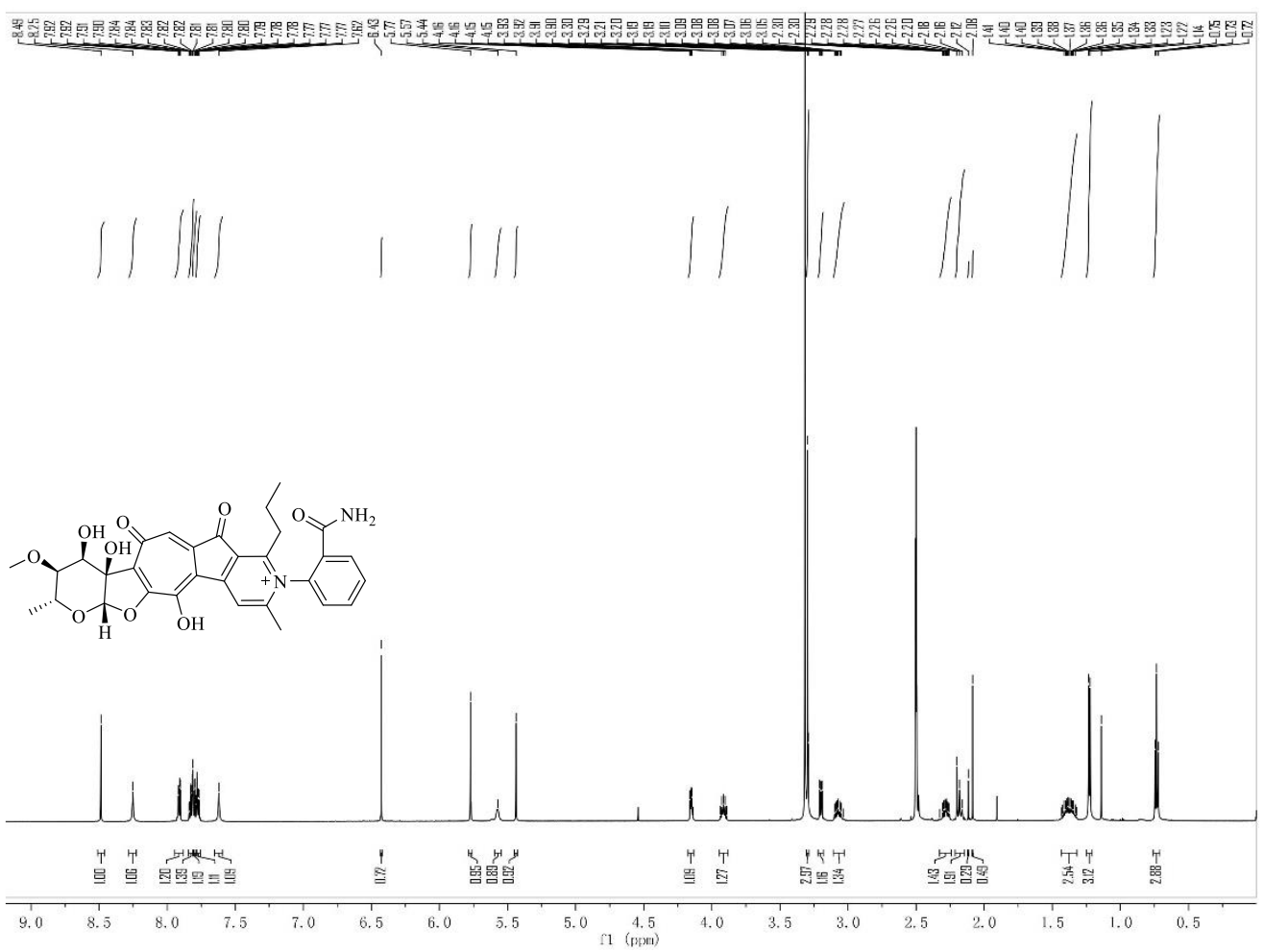

Figure S19. ${ }^{1} \mathrm{H}$ NMR spectrum of isarubrolone D (2).

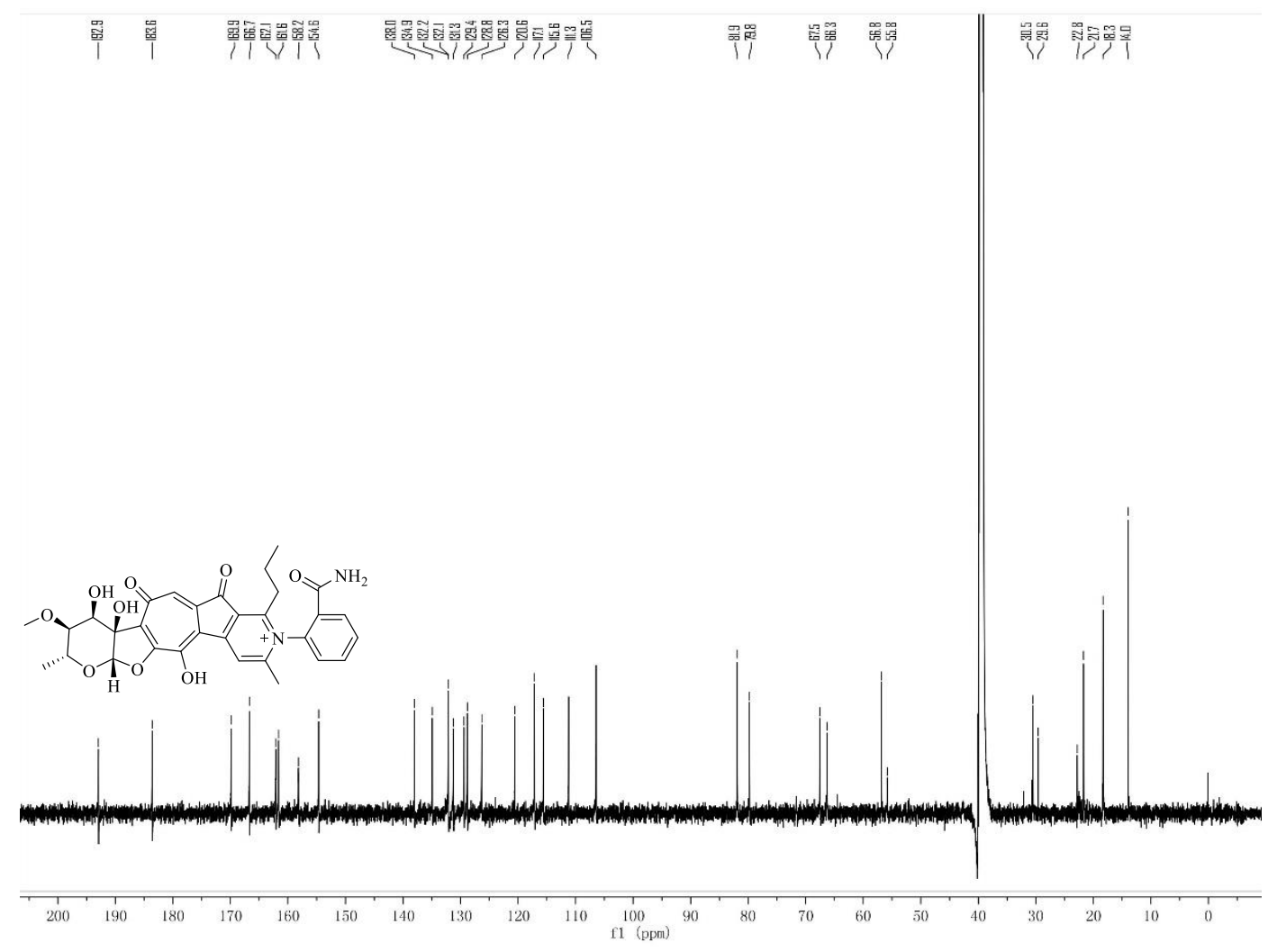

Figure S20. ${ }^{13} \mathrm{C}$ NMR spectrum of isarubrolone D (2). 
20180719 623-575-DMSO

DEPT9O DKSO D: $\backslash \backslash$ DATA2018 1

(n)

$20180719623-575-D M S O$

DLPT135 DUSO D: \ DATA2018 10

1
1

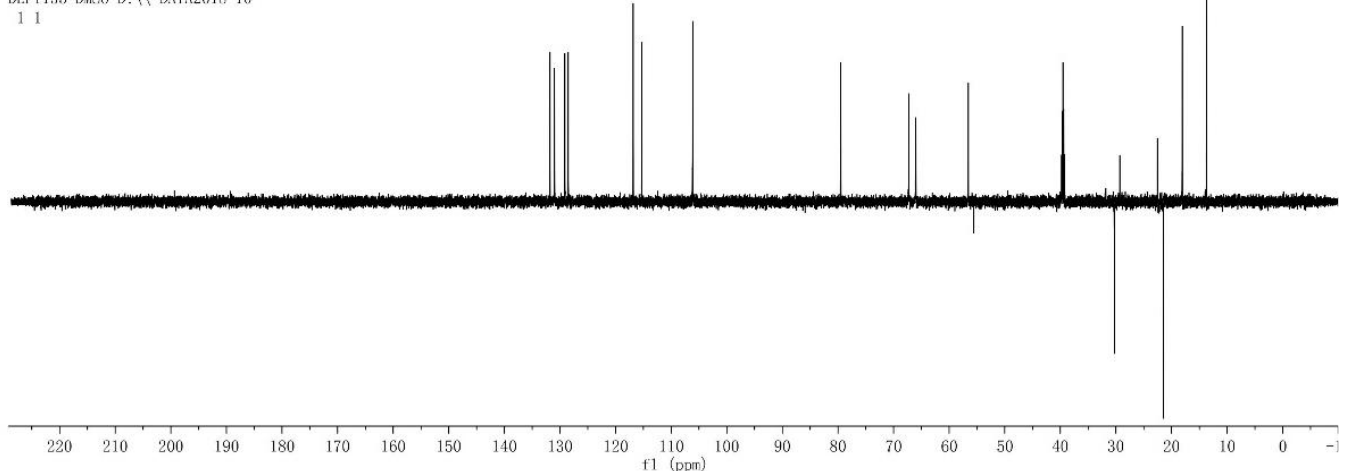

Figure S21. DEPT spectrum of isarubrolone D (2).

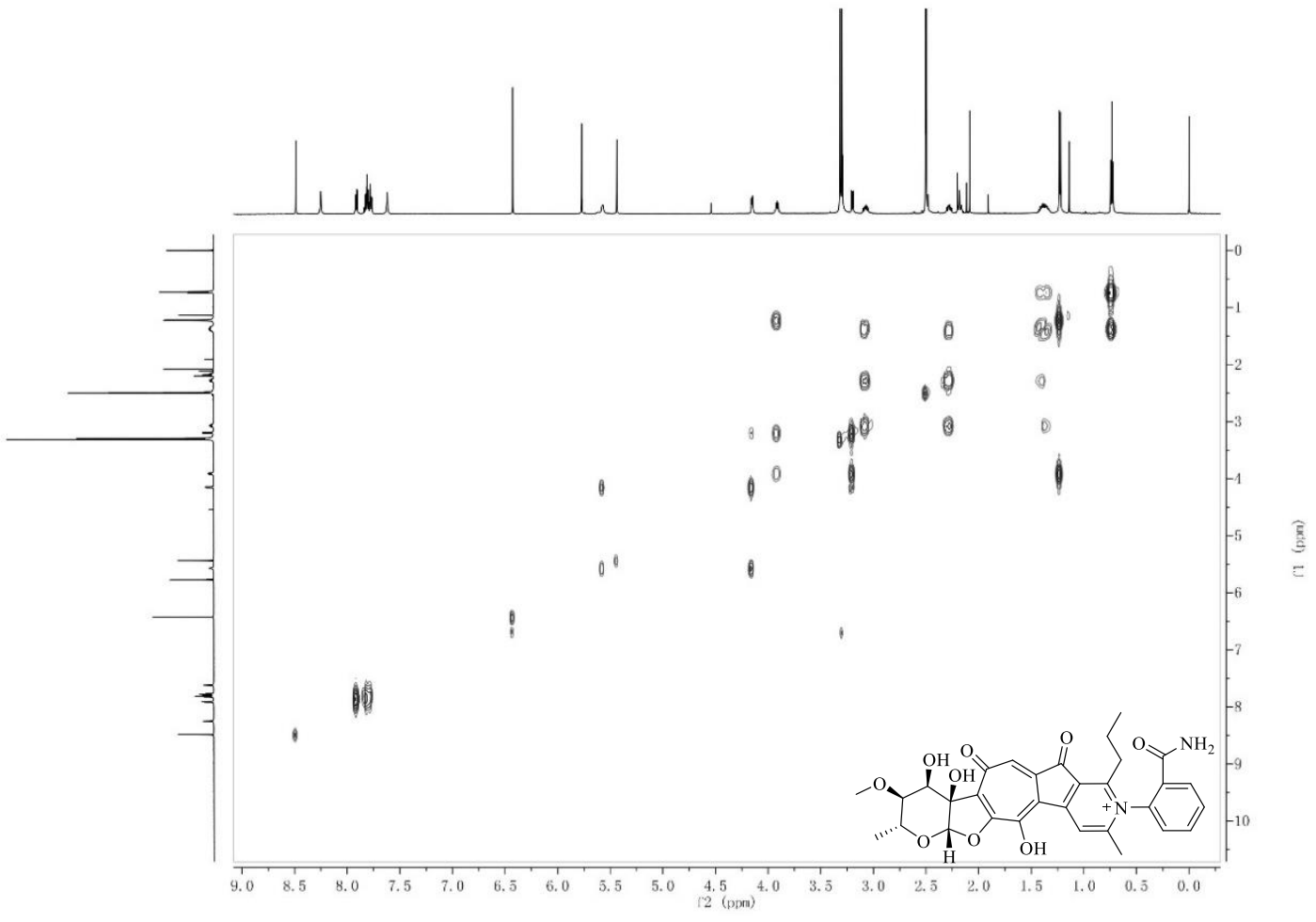

Figure S22. ${ }^{1} \mathrm{H}-{ }^{1} \mathrm{H}$ COSY spectrum of isarubrolone D (2). 


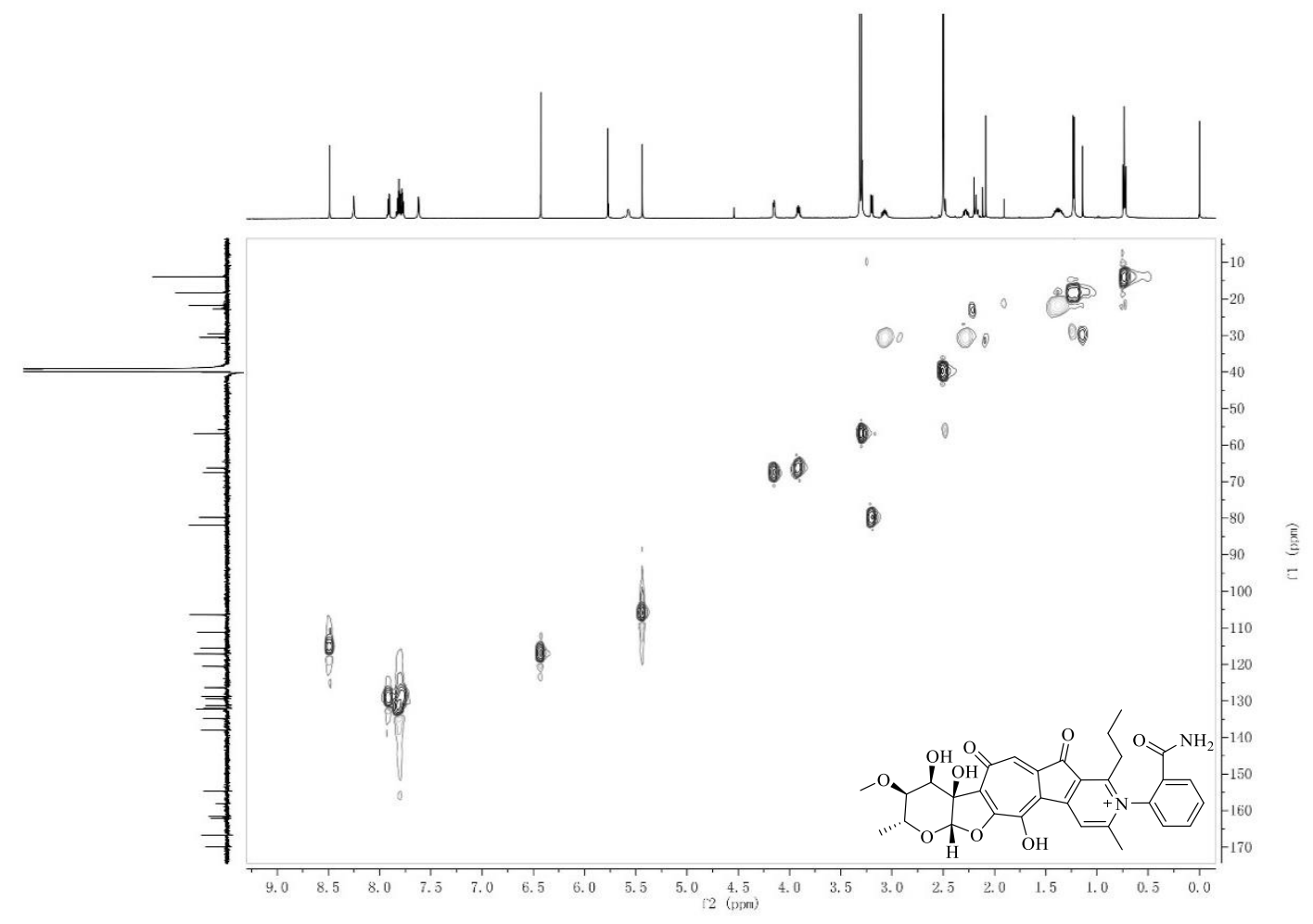

Figure S23. HSQC spectrum of isarubrolone D (2).

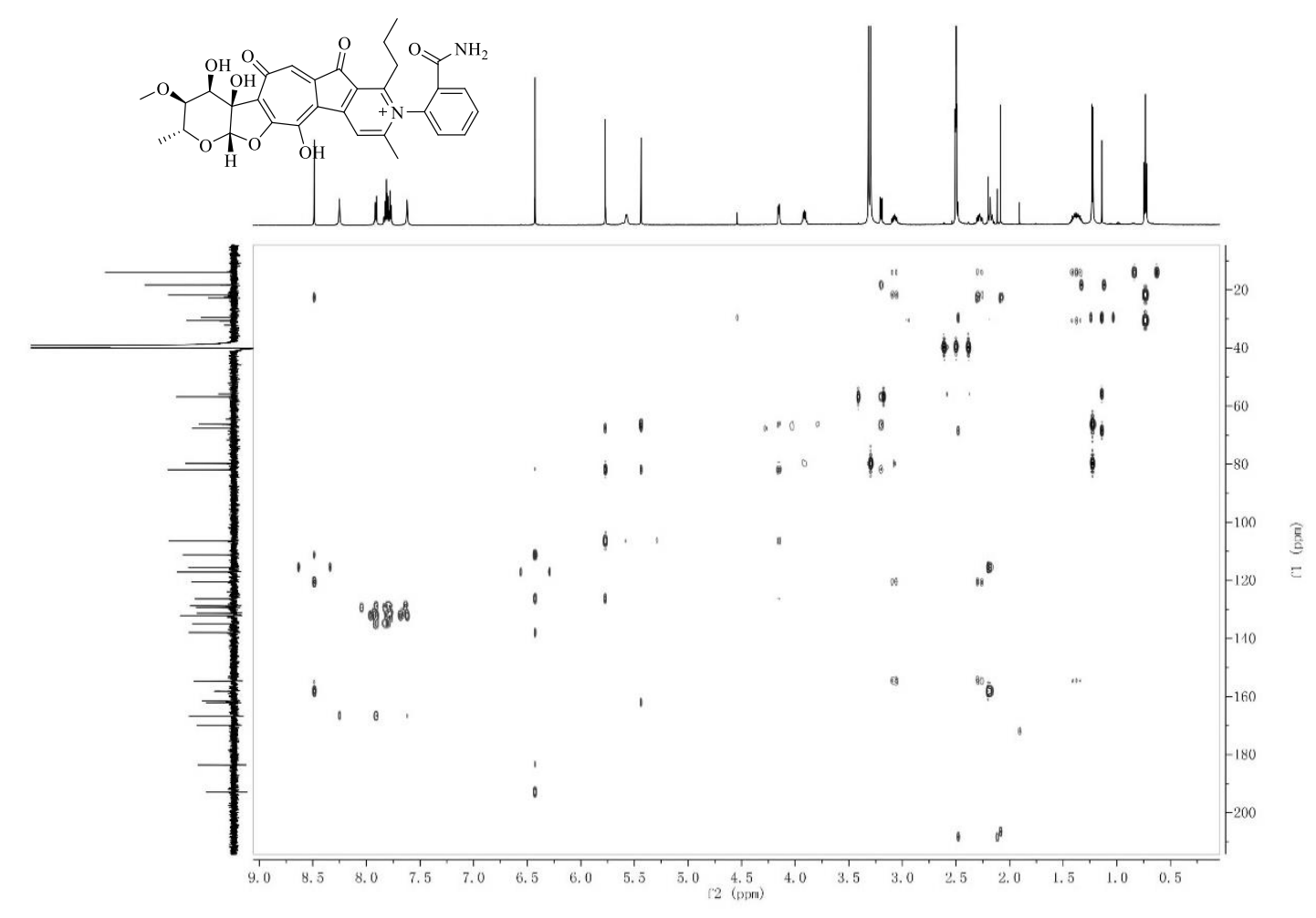

Figure S24. HMBC spectrum of isarubrolone D (2). 


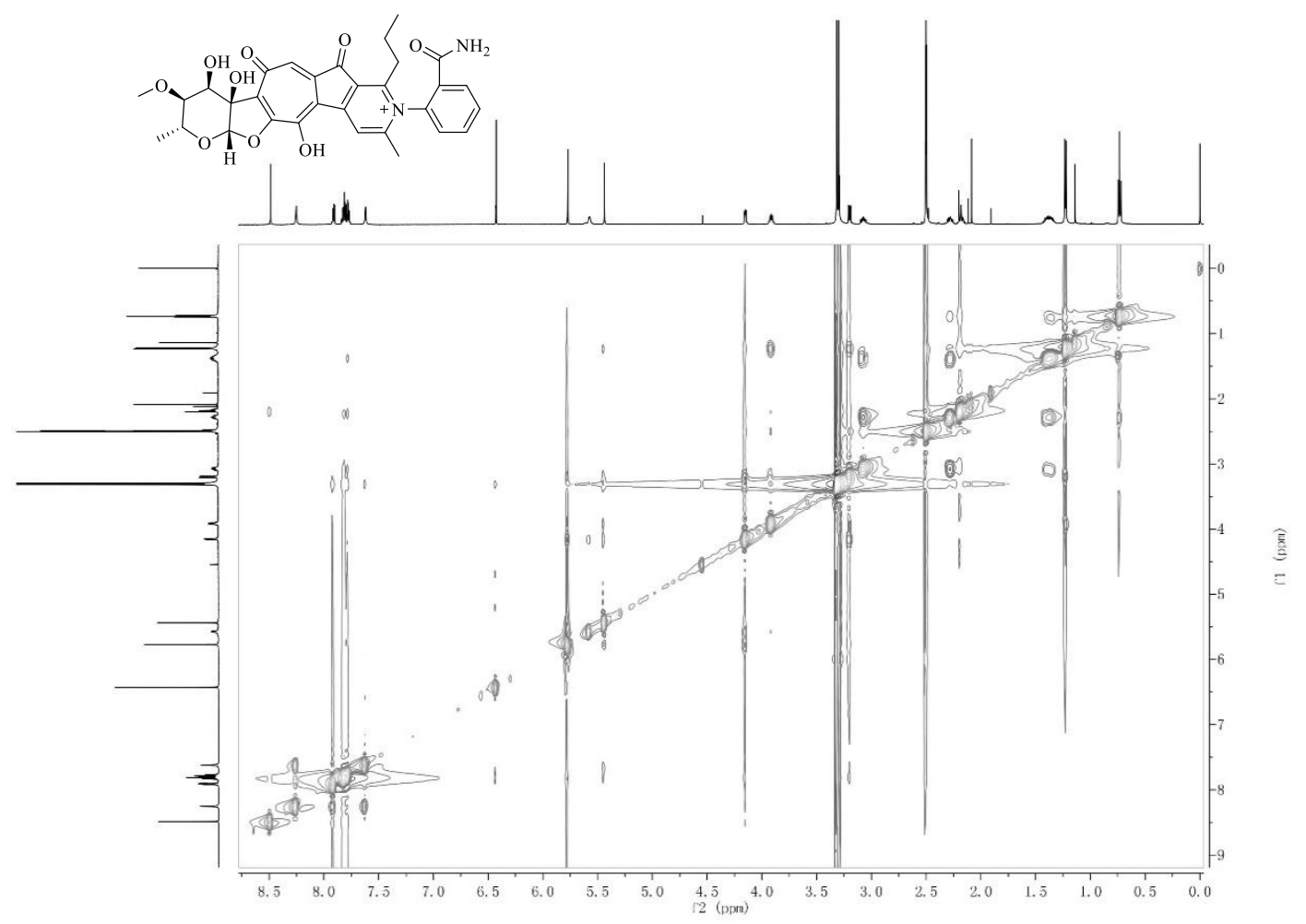

Figure S25. NOESY spectrum of isarubrolone D (2).

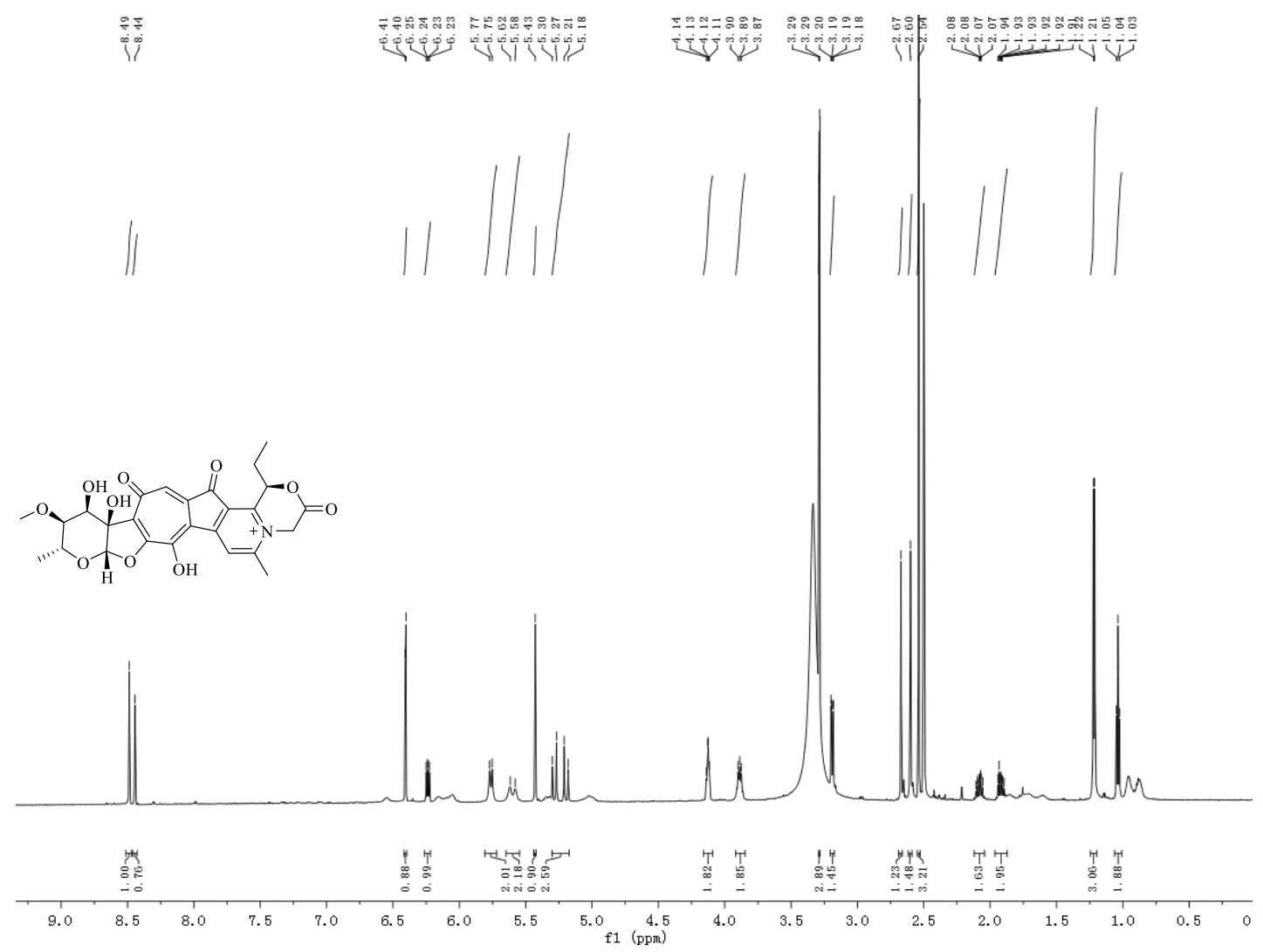

Figure S26. ${ }^{1} \mathrm{H}$ NMR spectrum of isarubrolone E (3). 

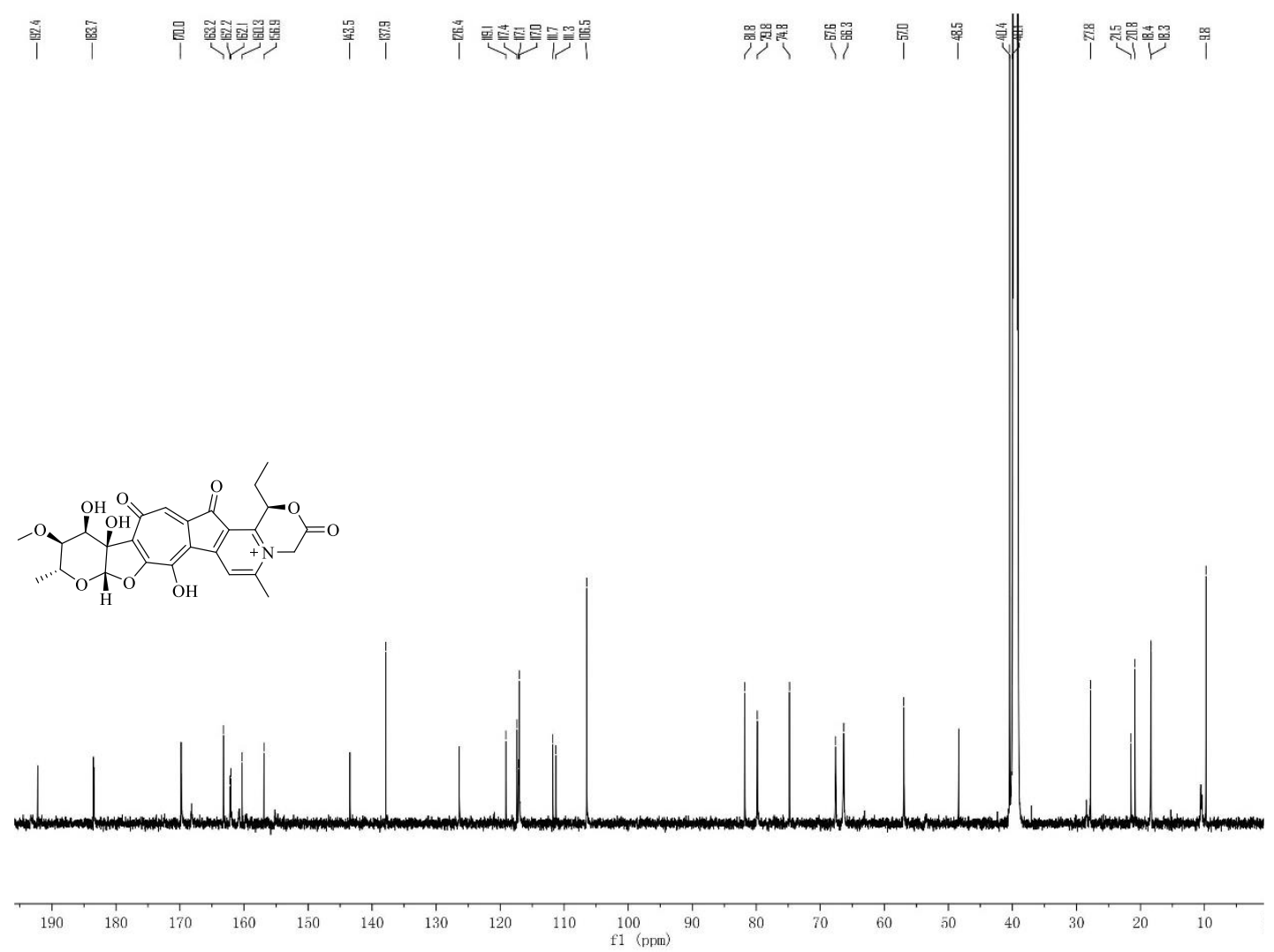

Figure S27. ${ }^{13} \mathrm{C}$ spectrum of isarubrolone E (3).

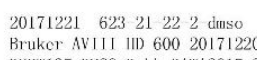

DE:PT135 DMSO D. D\DATA2017 31

22
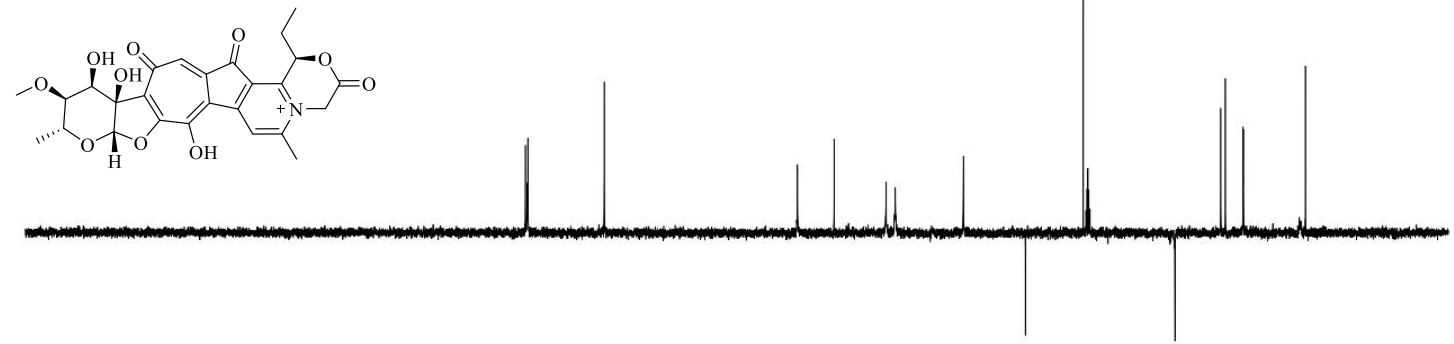

$20171221 \quad 623-21-22-2$-duss

Bruker AVIII HD 60020171220
DEPT90 DMSO D: $\backslash \backslash$ DATA2017

11
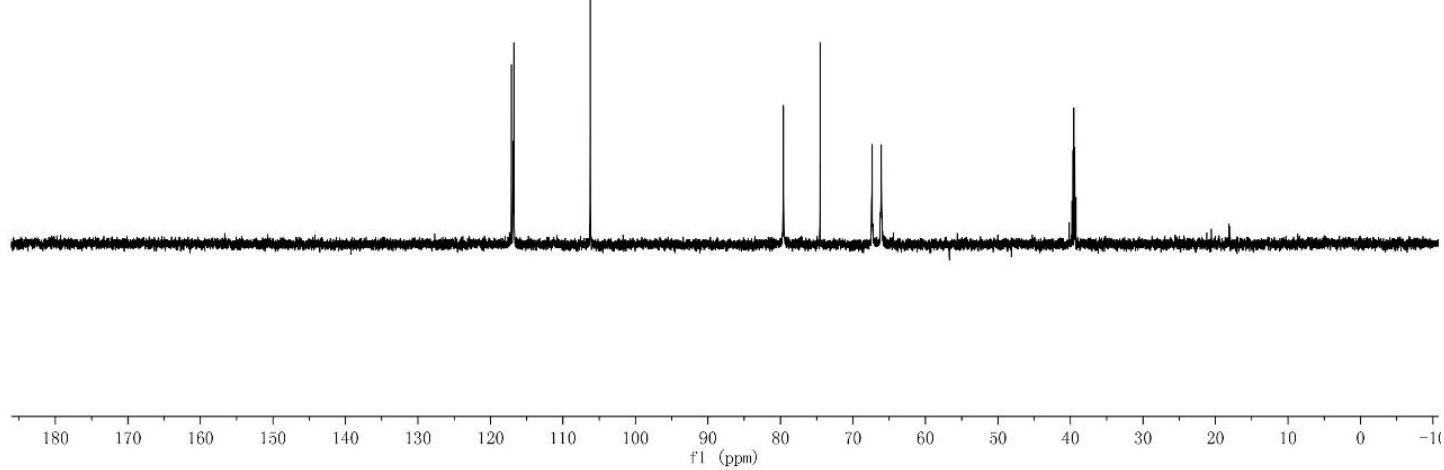

Figure S28. DEPT spectrum of isarubrolone E (3). 


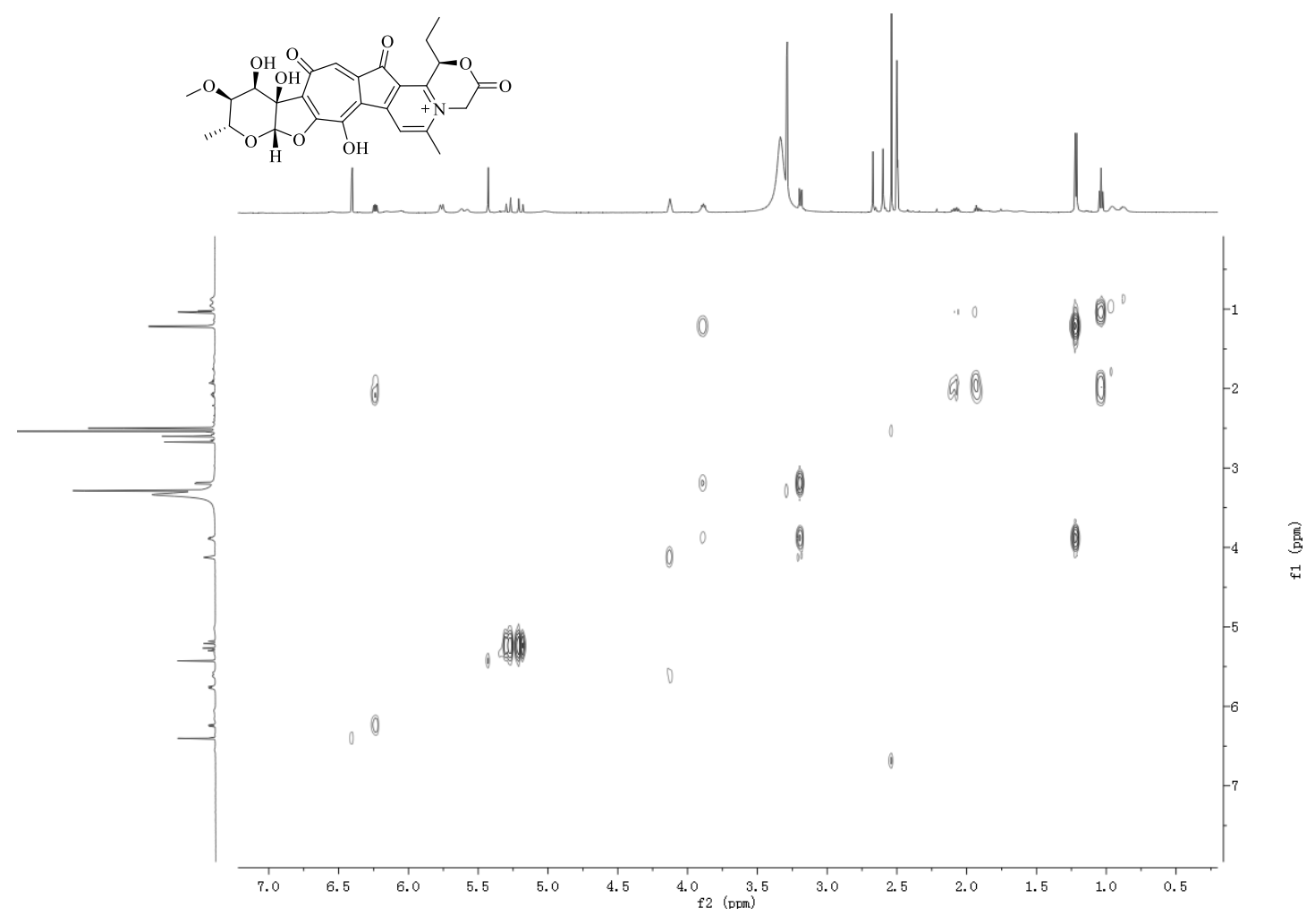

Figure S29. ${ }^{1} \mathrm{H}-{ }^{1} \mathrm{H}$ COSY spectrum of isarubrolone E (3).

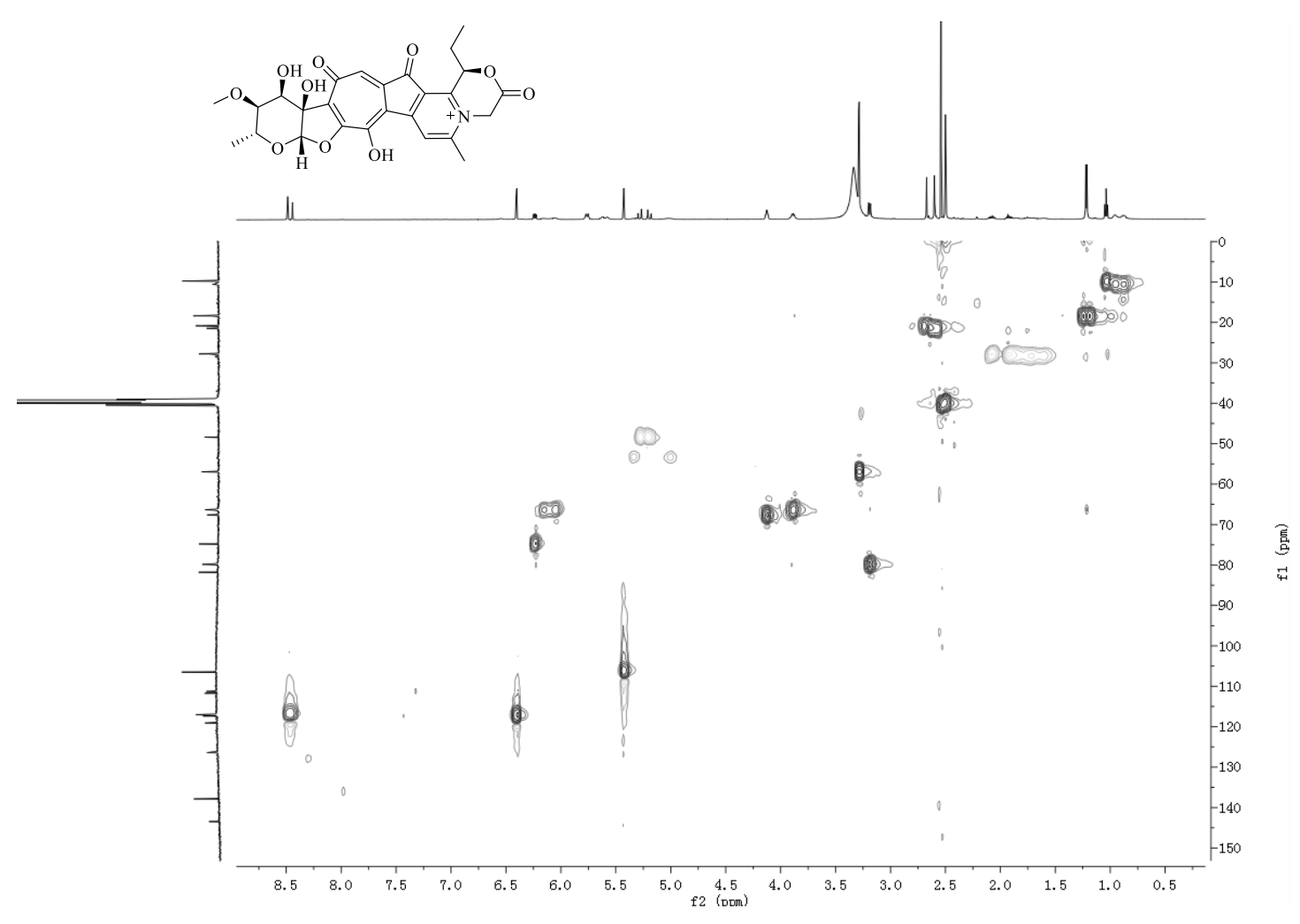

Figure S30. HSQC spectrum of isarubrolone E (3). 


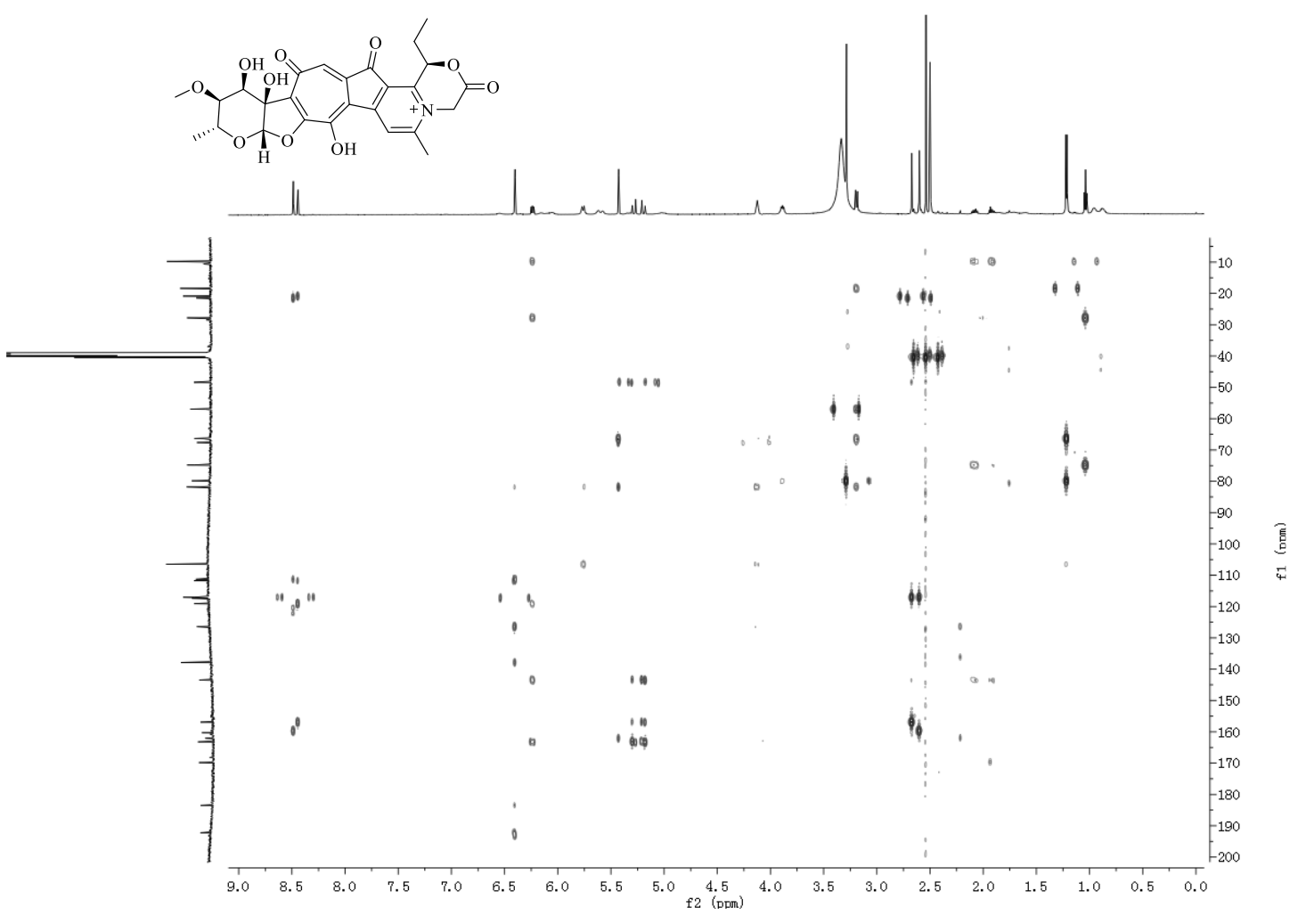

Figure S31. HMBC spectrum of isarubrolone E (3).

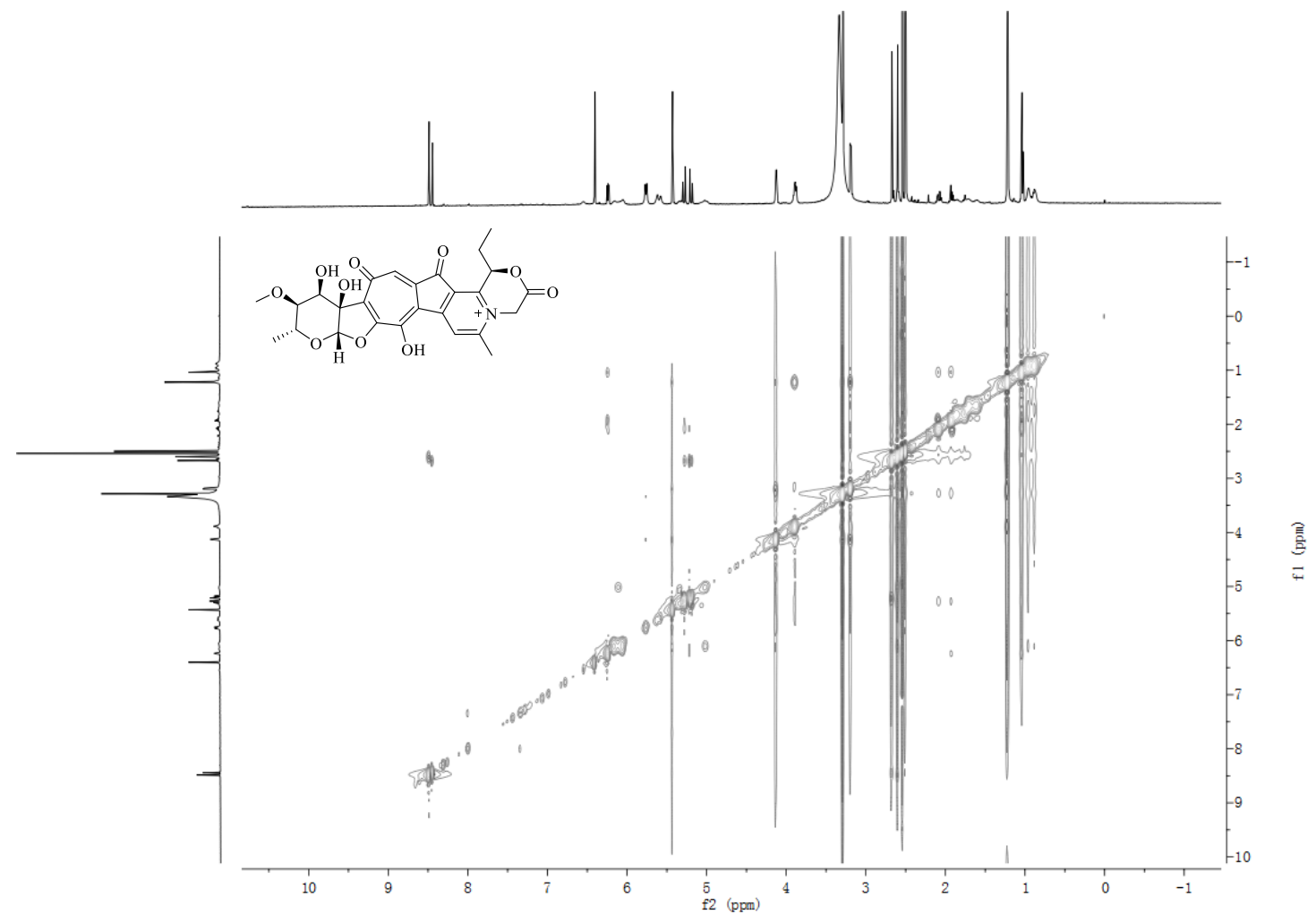

Figure S32. NOESY spectrum of isarubrolone E (3). 


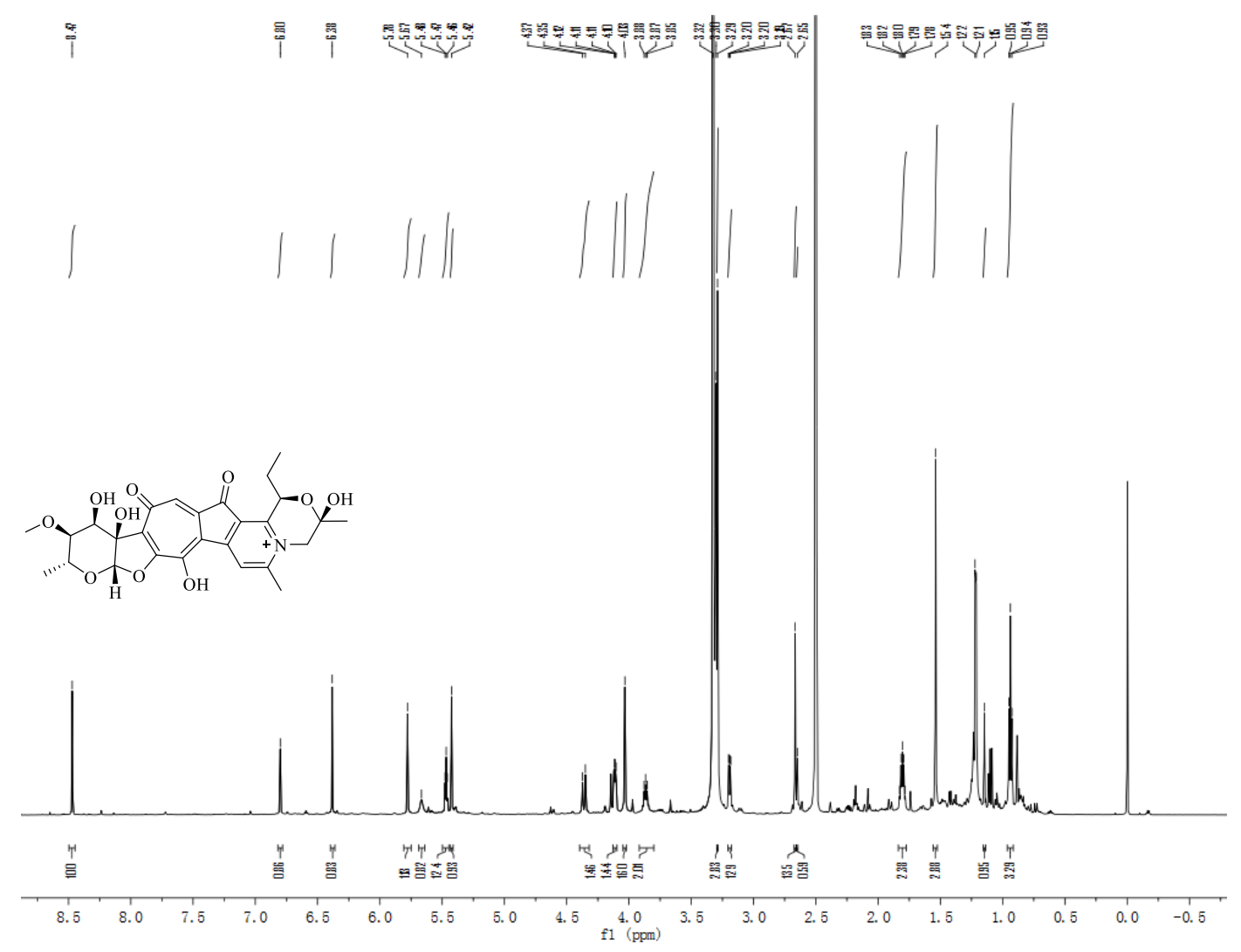

Figure S33. ${ }^{1} \mathrm{H}$ NMR spectrum of isarubrolone F (4).

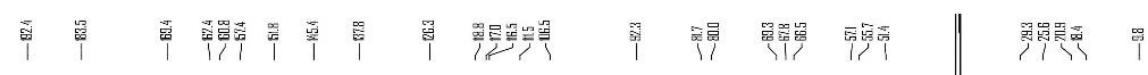
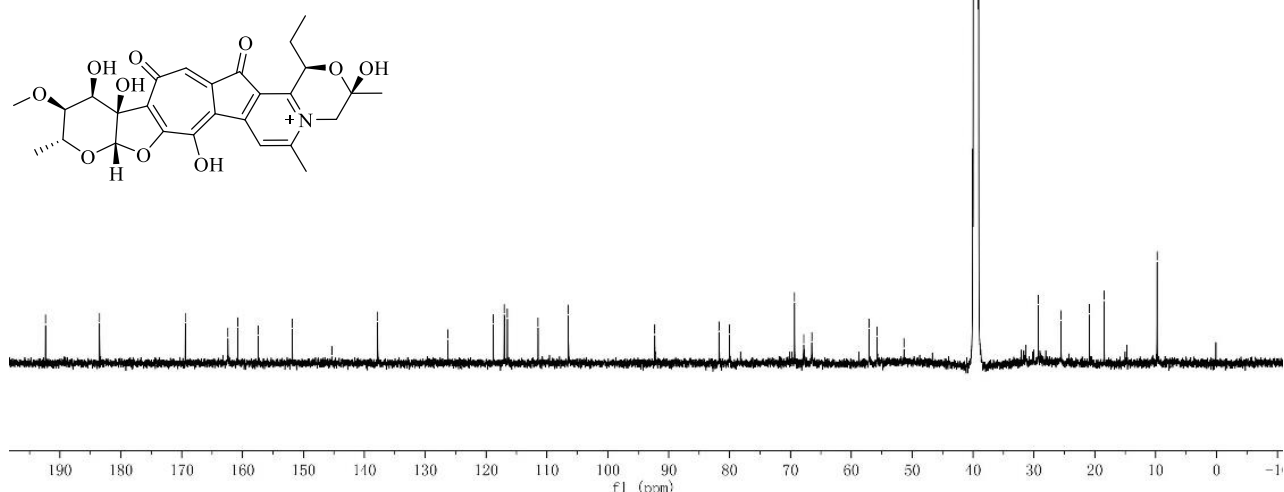

Figure S34. ${ }^{13} \mathrm{C}$ NMR spectrum of isarubrolone $\mathrm{F}(4)$. 
DEPT_01

VNS-600 DEPT 623-527 IN dmso Nov 62017

(n)

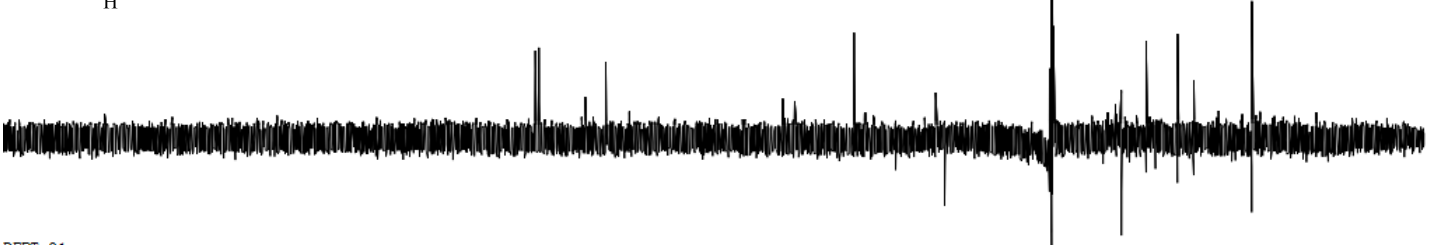

DEPT_01
VNS-600 DEPT 623-527 IN dmso Nov 62017
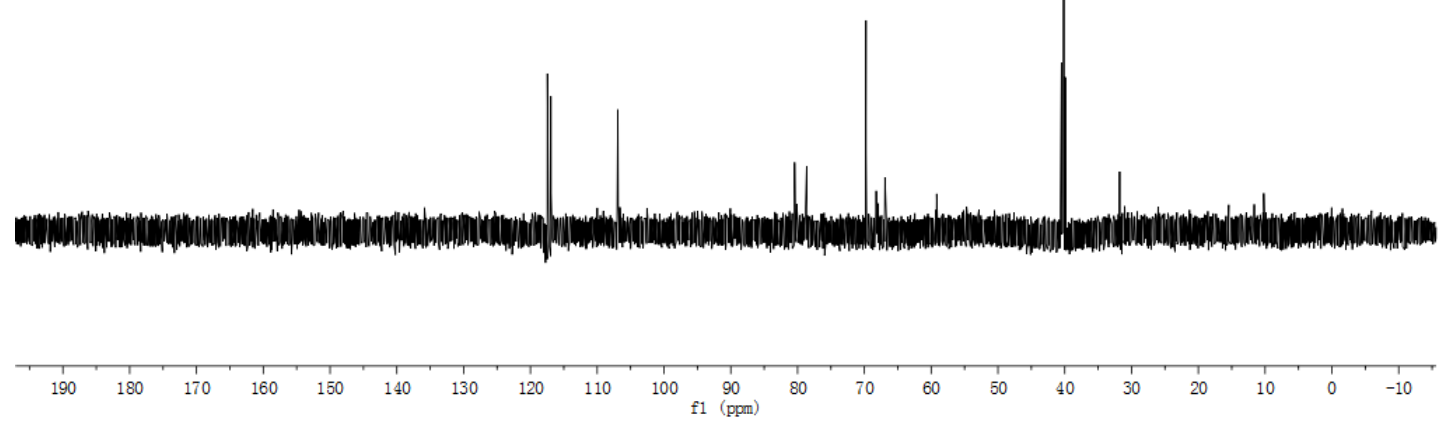

Figure S35. DEPT NMR spectrum of isarubrolone $F(4)$.

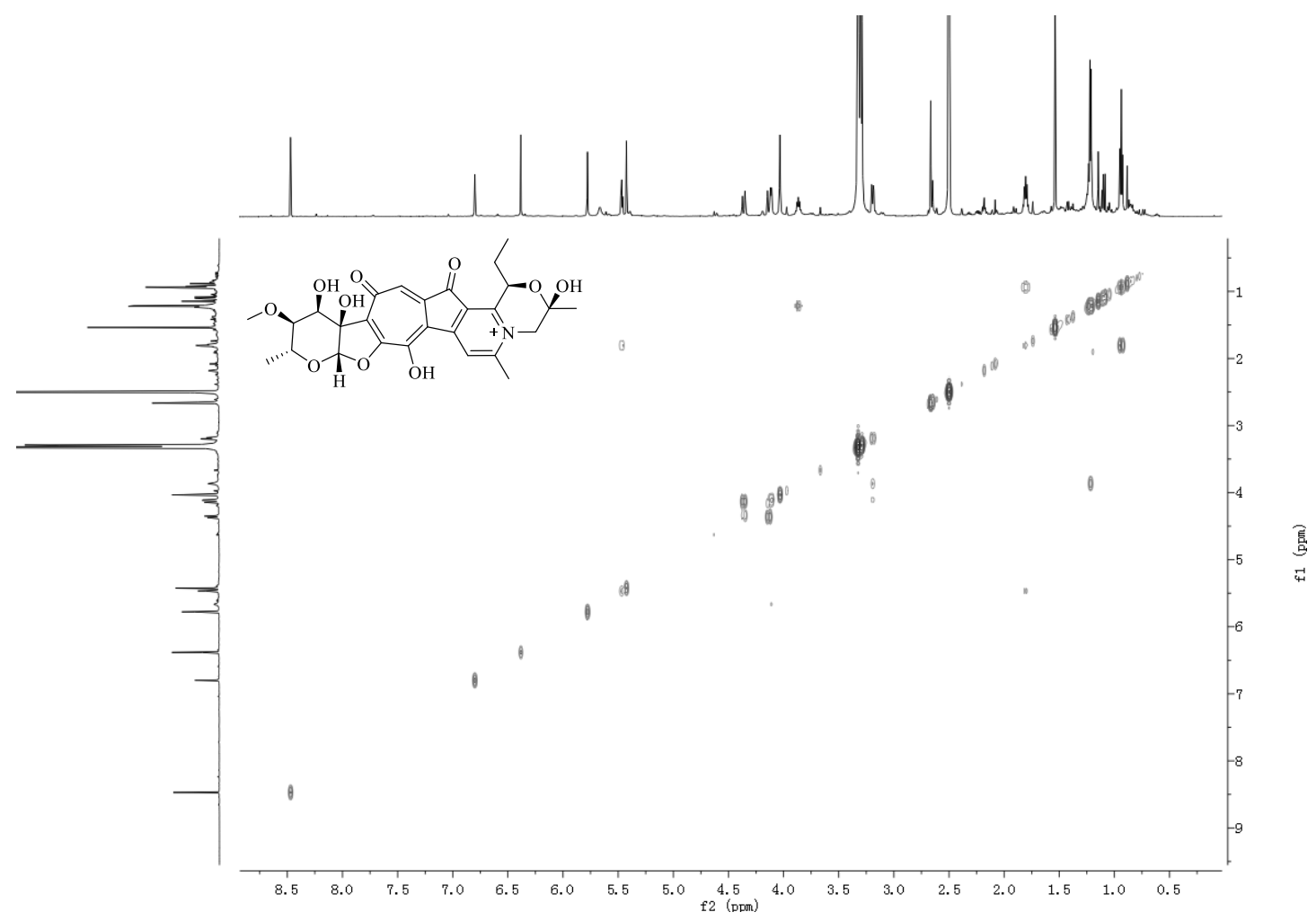

Figure S36. ${ }^{1} \mathrm{H}-{ }^{1} \mathrm{H}$ COSY spectrum of isarubrolone $\mathrm{F}(4)$. 


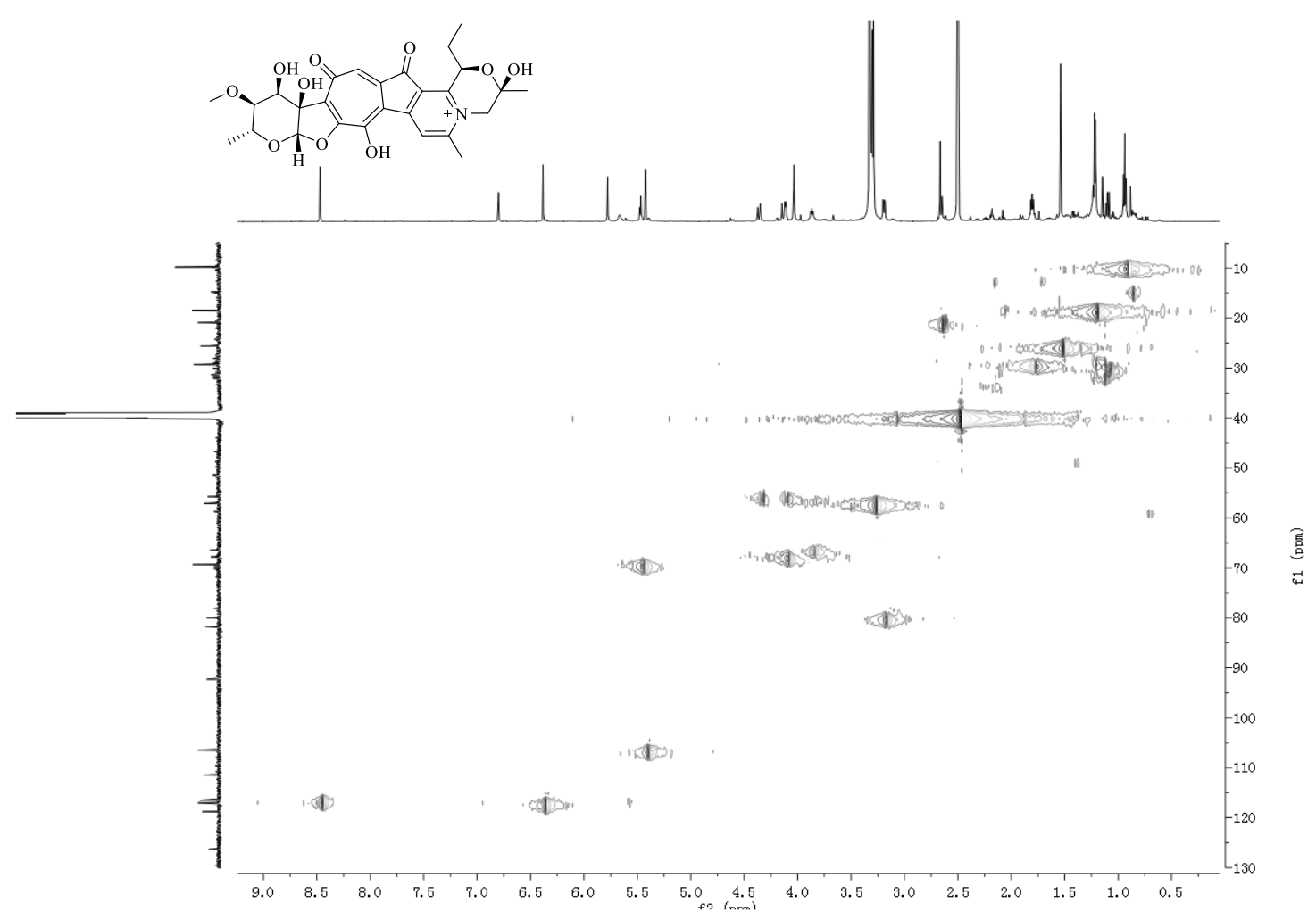

Figure S37. HSQC spectrum of isarubrolone F (4).

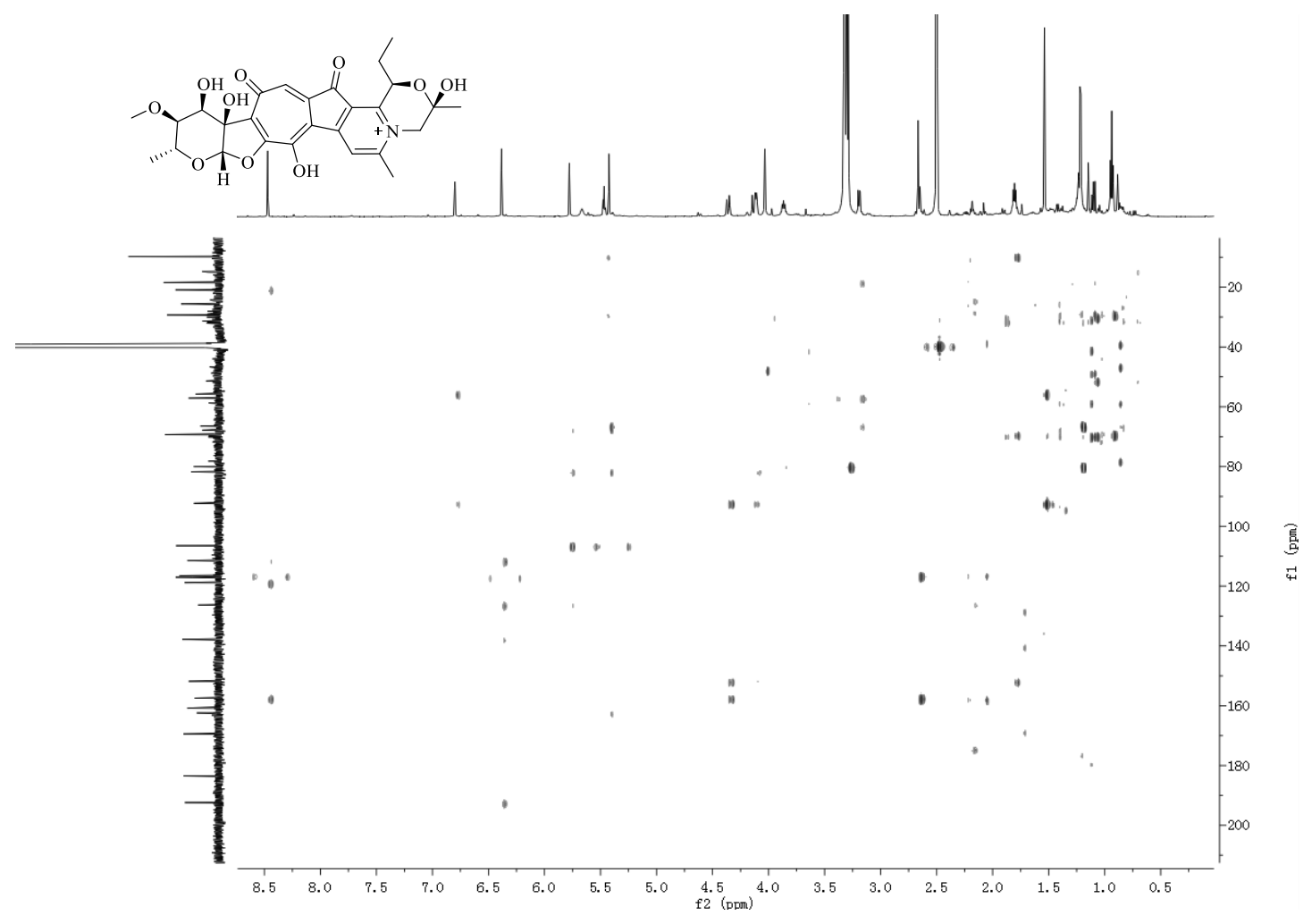

Figure S38. HMBC spectrum of isarubrolone F (4). 


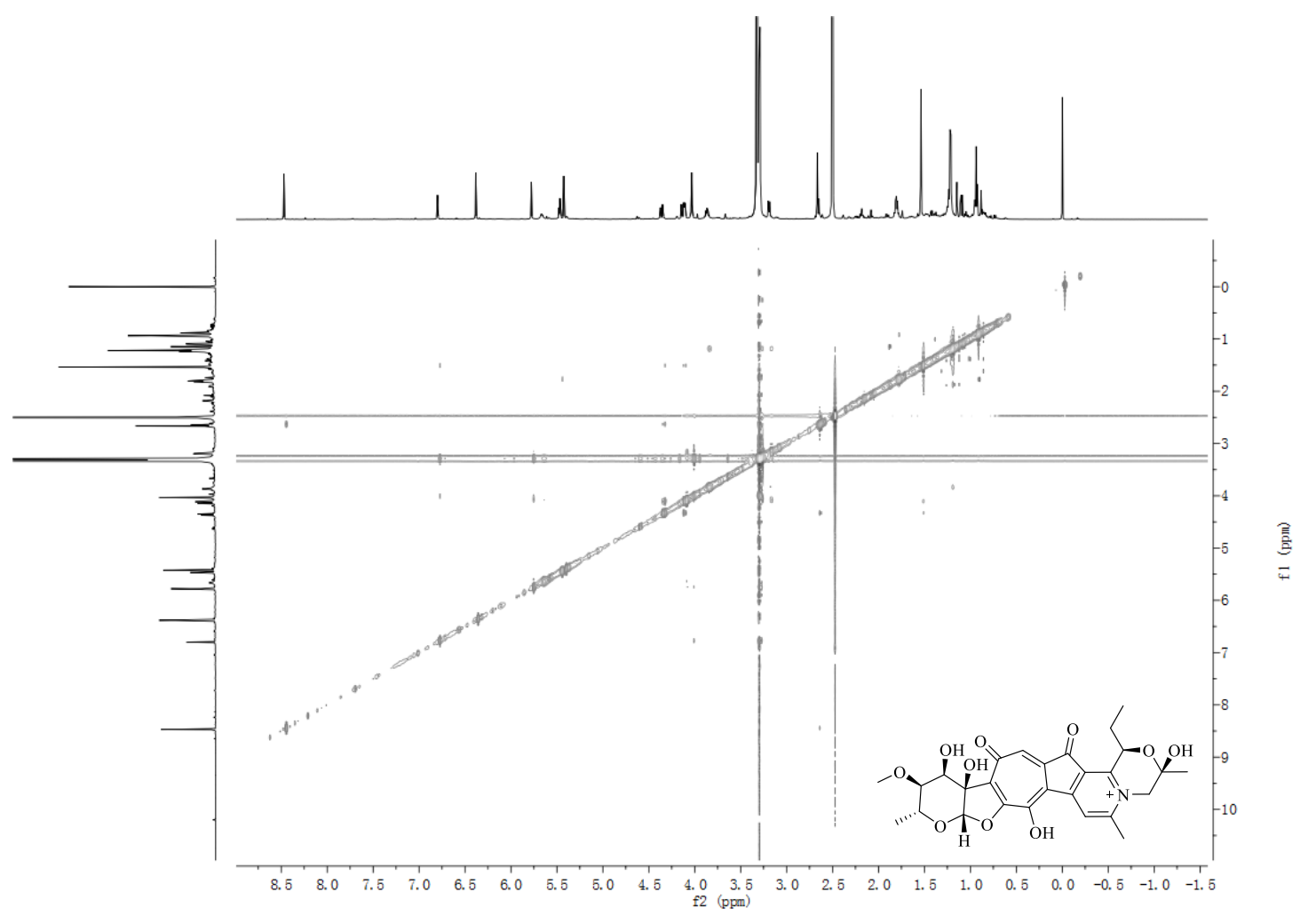

Figure S39. NOESY spectrum of isarubrolone F (4).

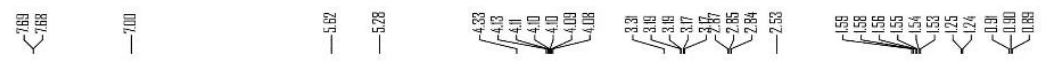
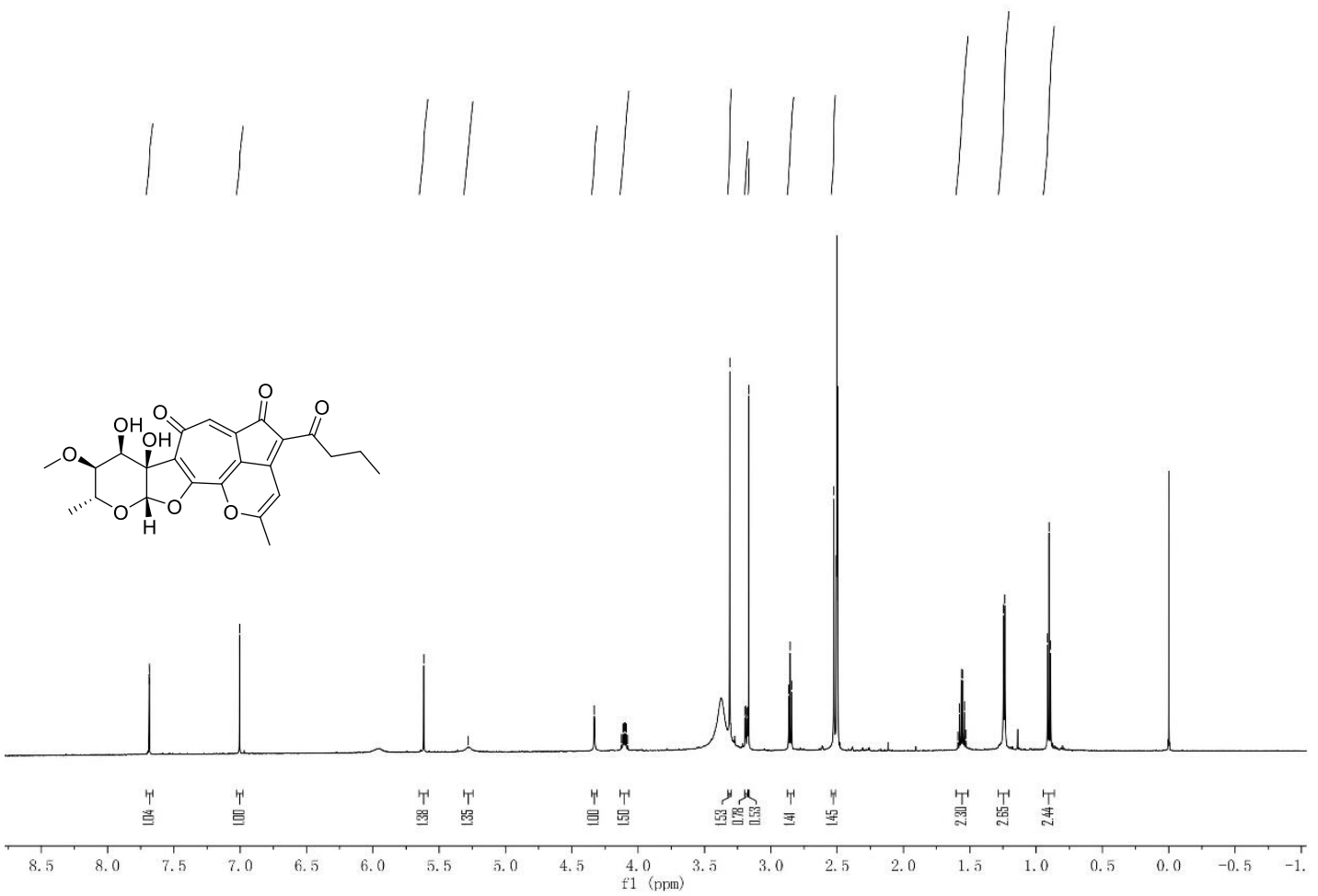

Figure S40. ${ }^{1} \mathrm{H}$ NMR spectrum of isatropolone A (5). 


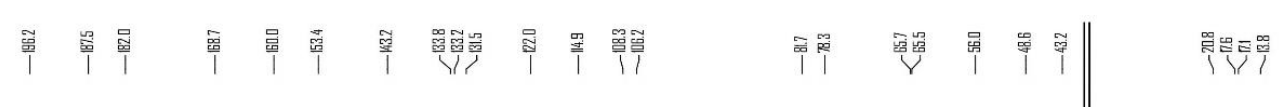
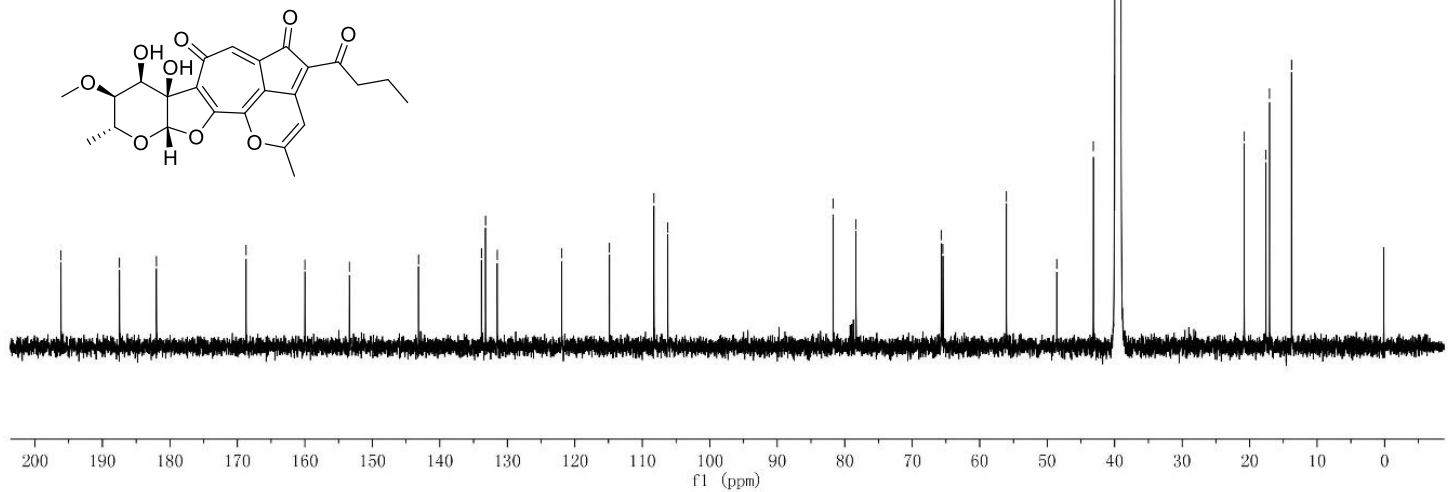

Figure S41. ${ }^{13} \mathrm{C}$ NMR spectrum of isatropolone A (5).

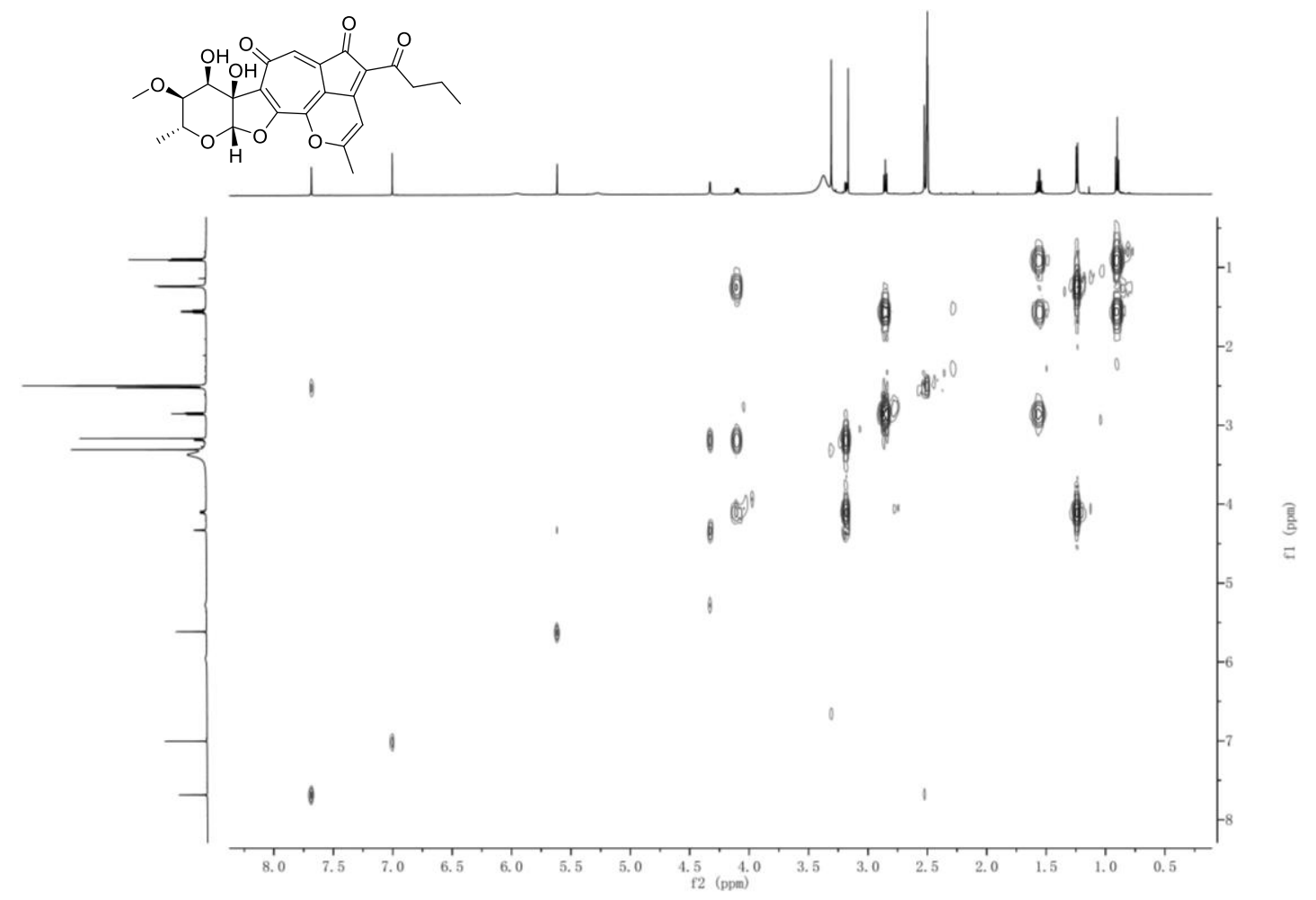

Figure S42. ${ }^{1} \mathrm{H}-{ }^{1} \mathrm{HCOSY}$ spectrum of isatropolone A (5). 


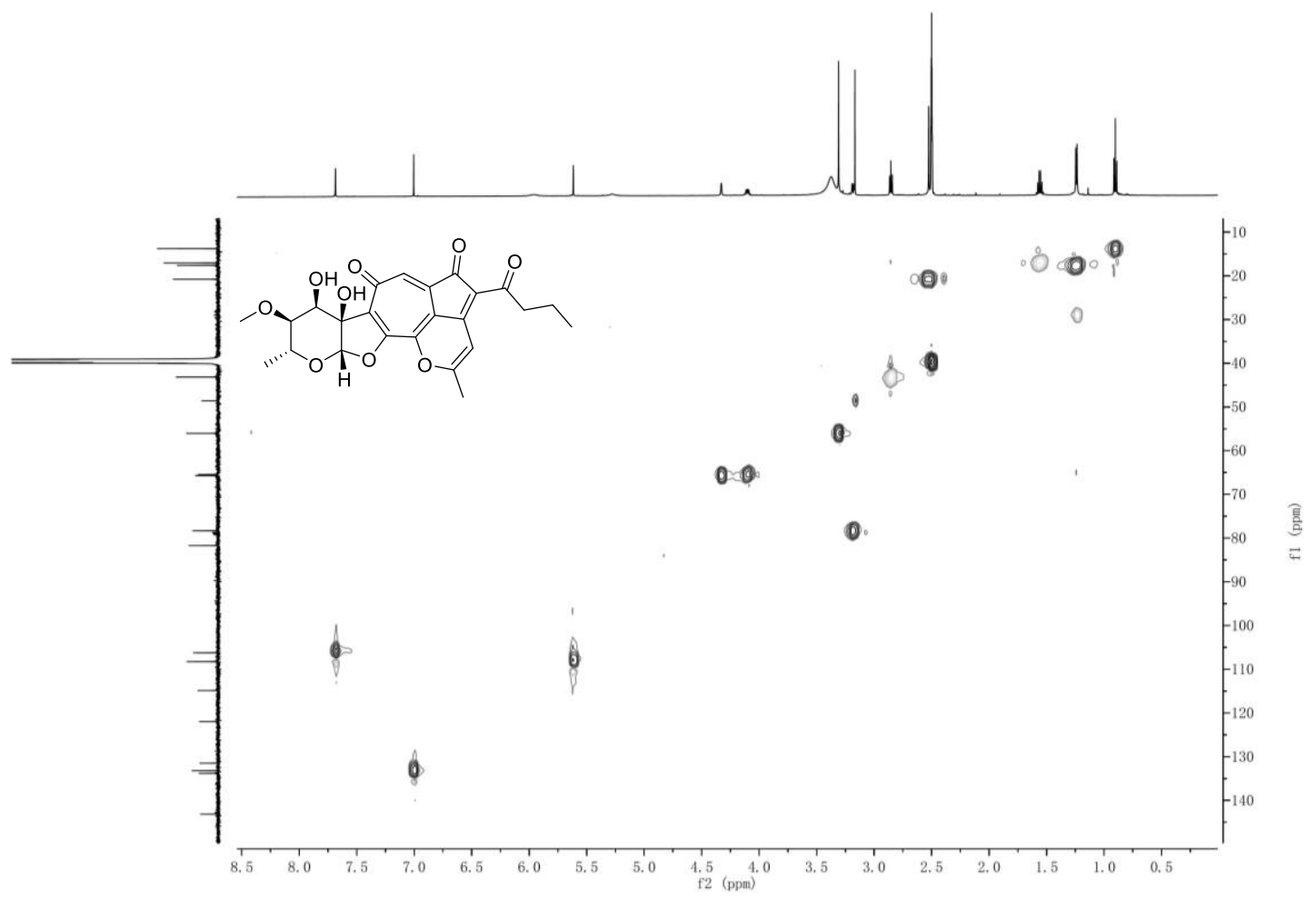

Figure S43. HSQC spectrum of isatropolone A (5).

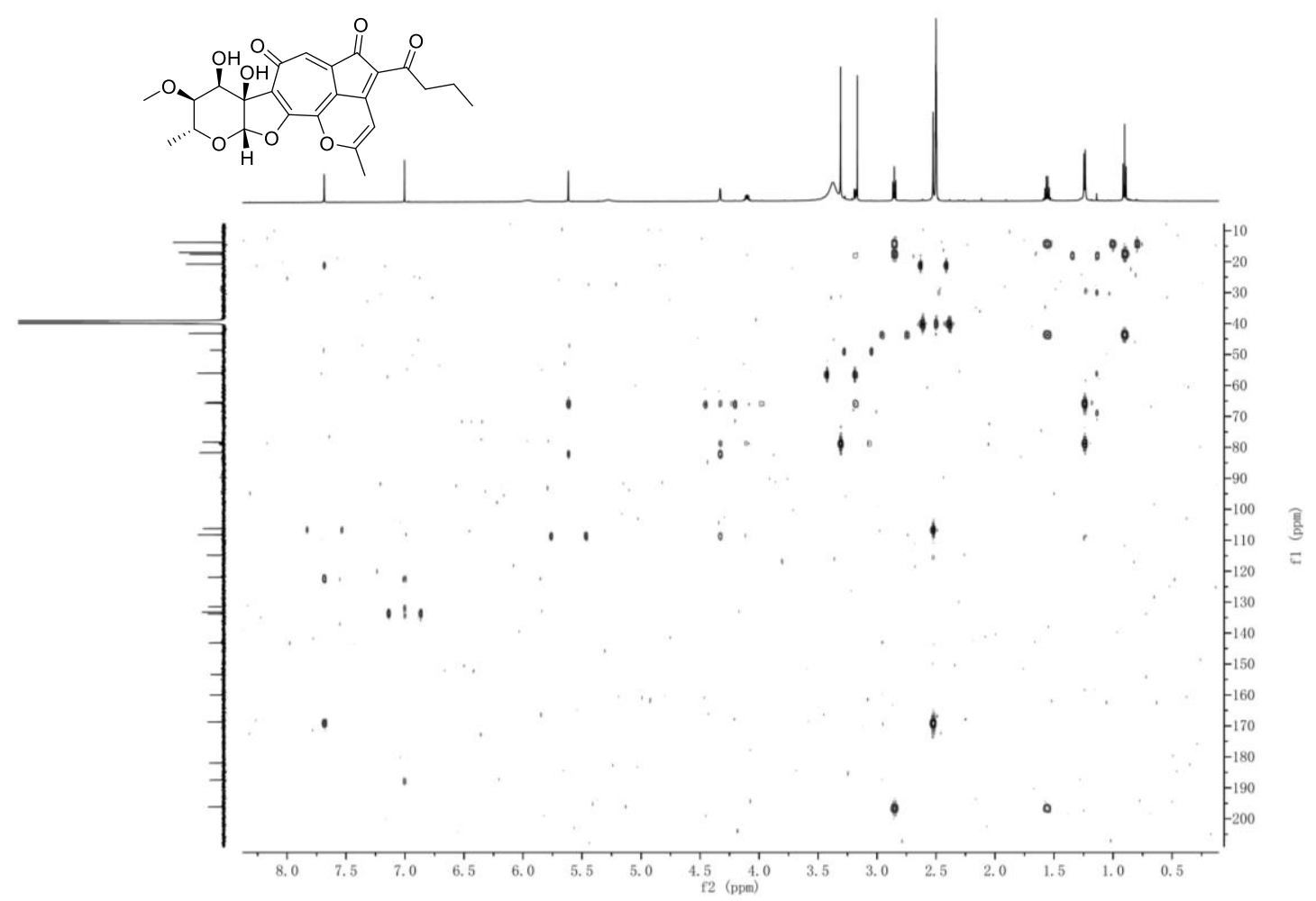

Figure S44. HMBC spectrum of isatropolone A (5). 


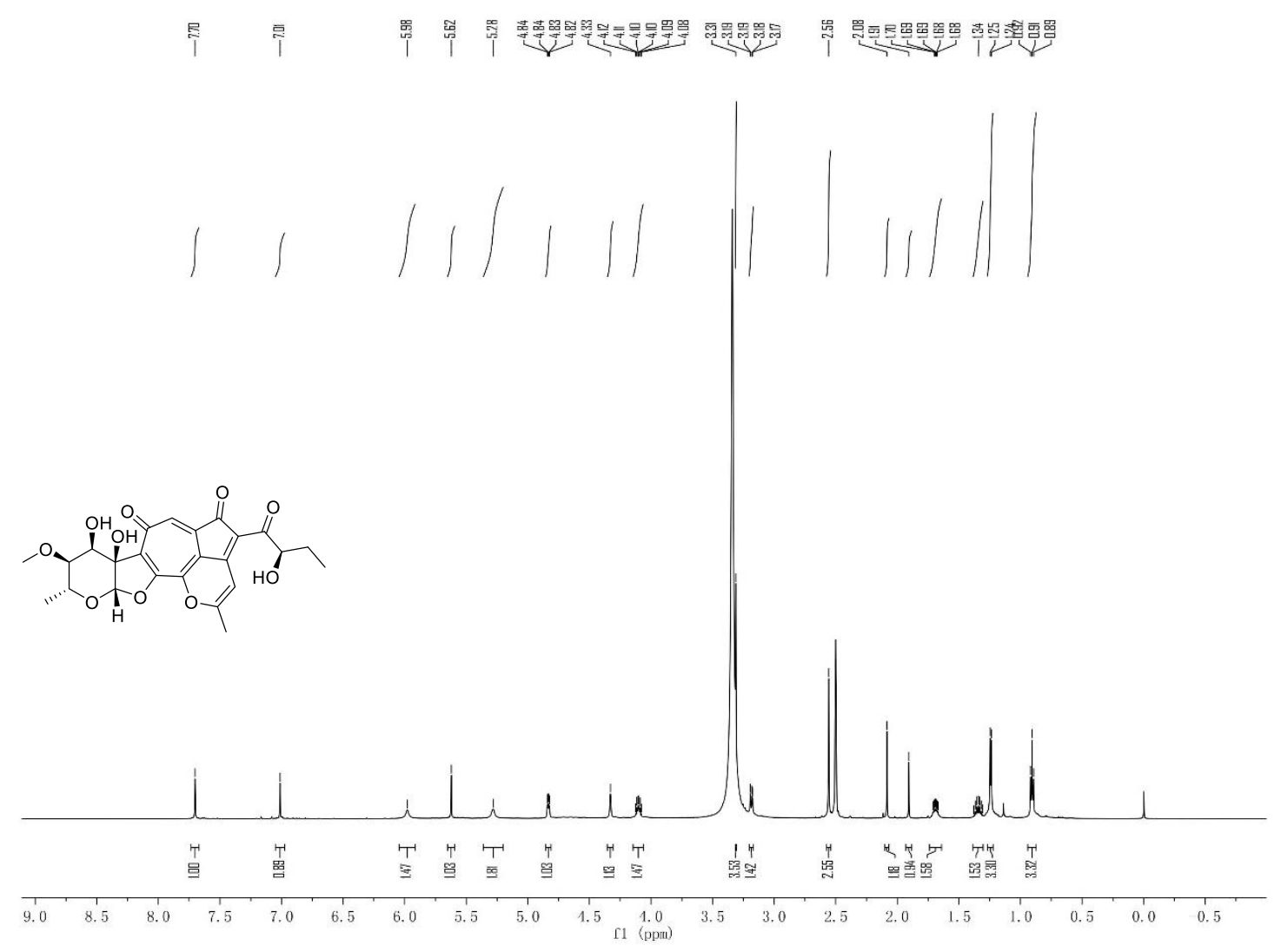

Figure S45. ${ }^{1} \mathrm{H}$ NMR spectrum of isatropolone $\mathrm{C}(\mathbf{6})$.
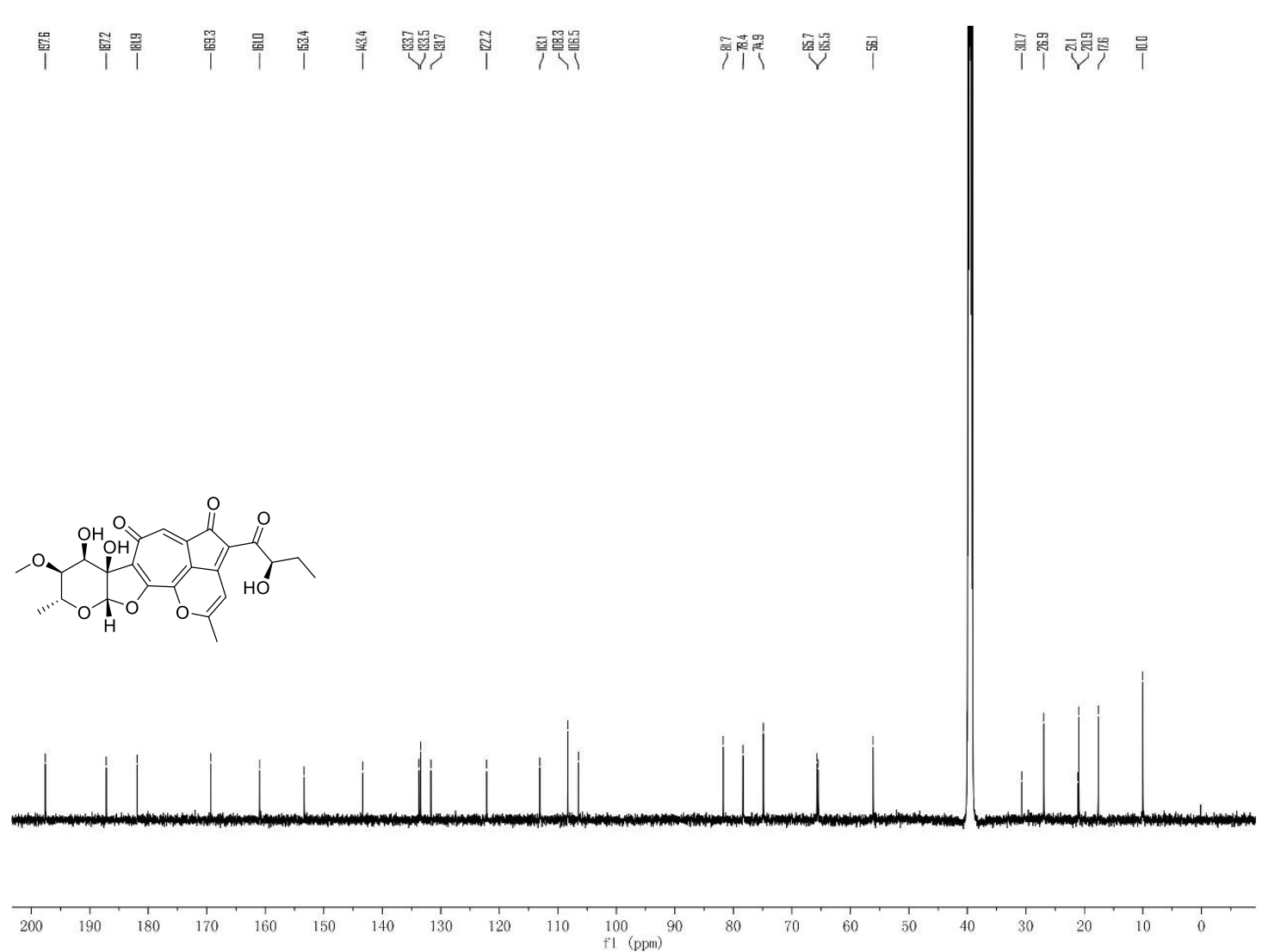

Figure S46. ${ }^{13} \mathrm{C}$ NMR spectrum of isatropolone C (6). 


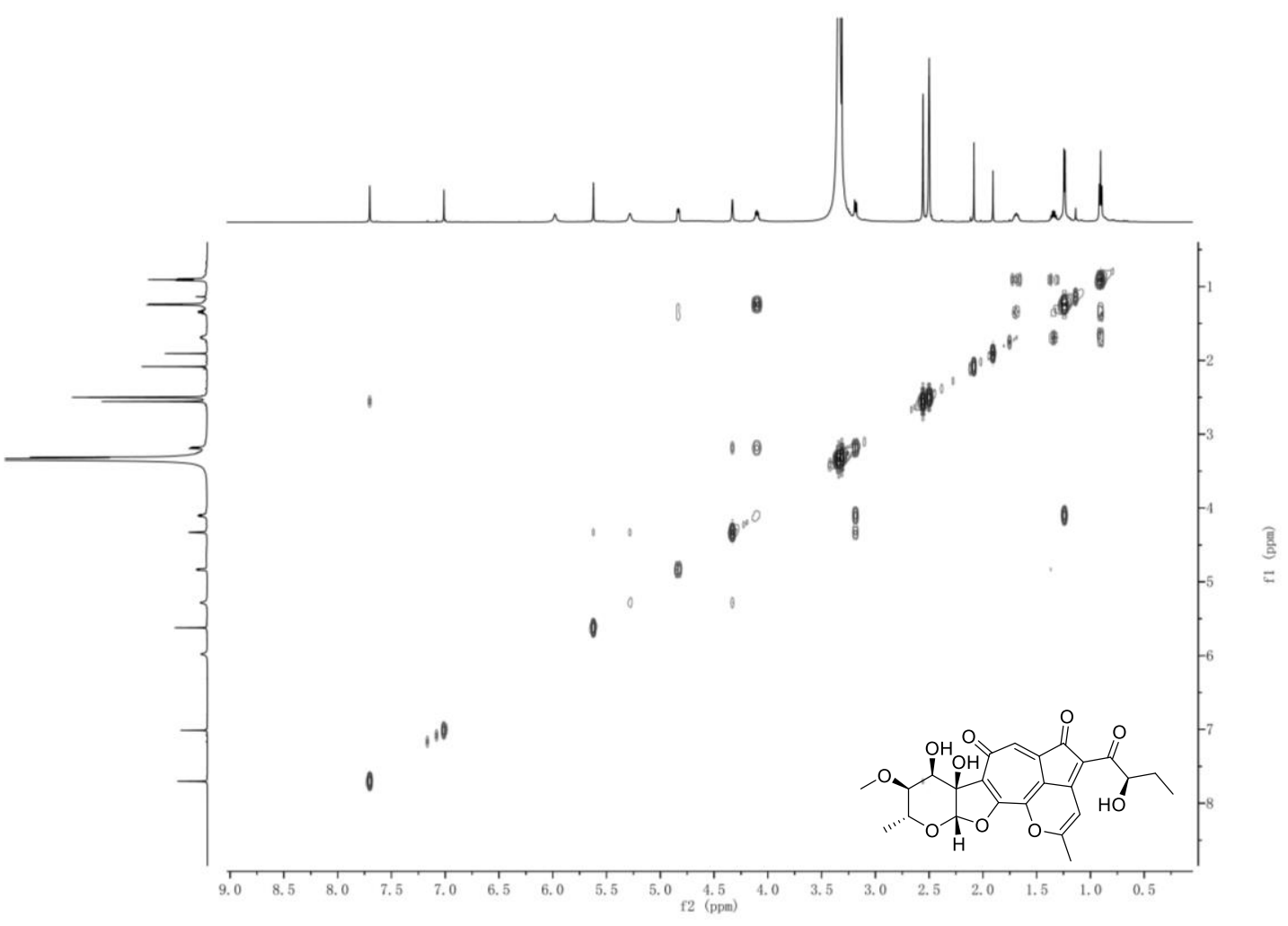

Figure S47. ${ }^{1} \mathrm{H}-{ }^{1} \mathrm{H}$ COSY spectrum of isatropolone $\mathrm{C}(\mathbf{6})$.

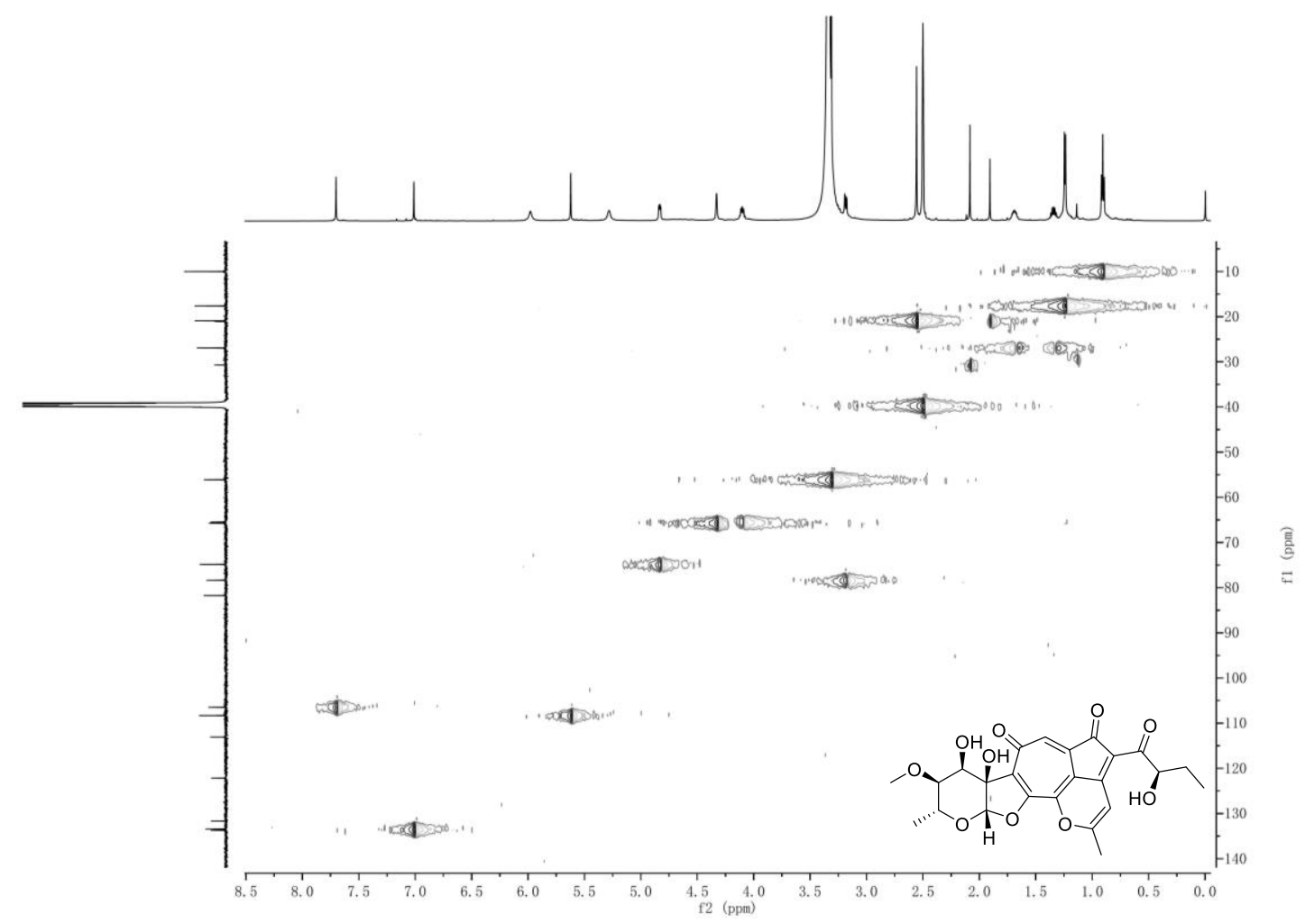

Figure S48. HSQC spectrum of isatropolone C (6). 


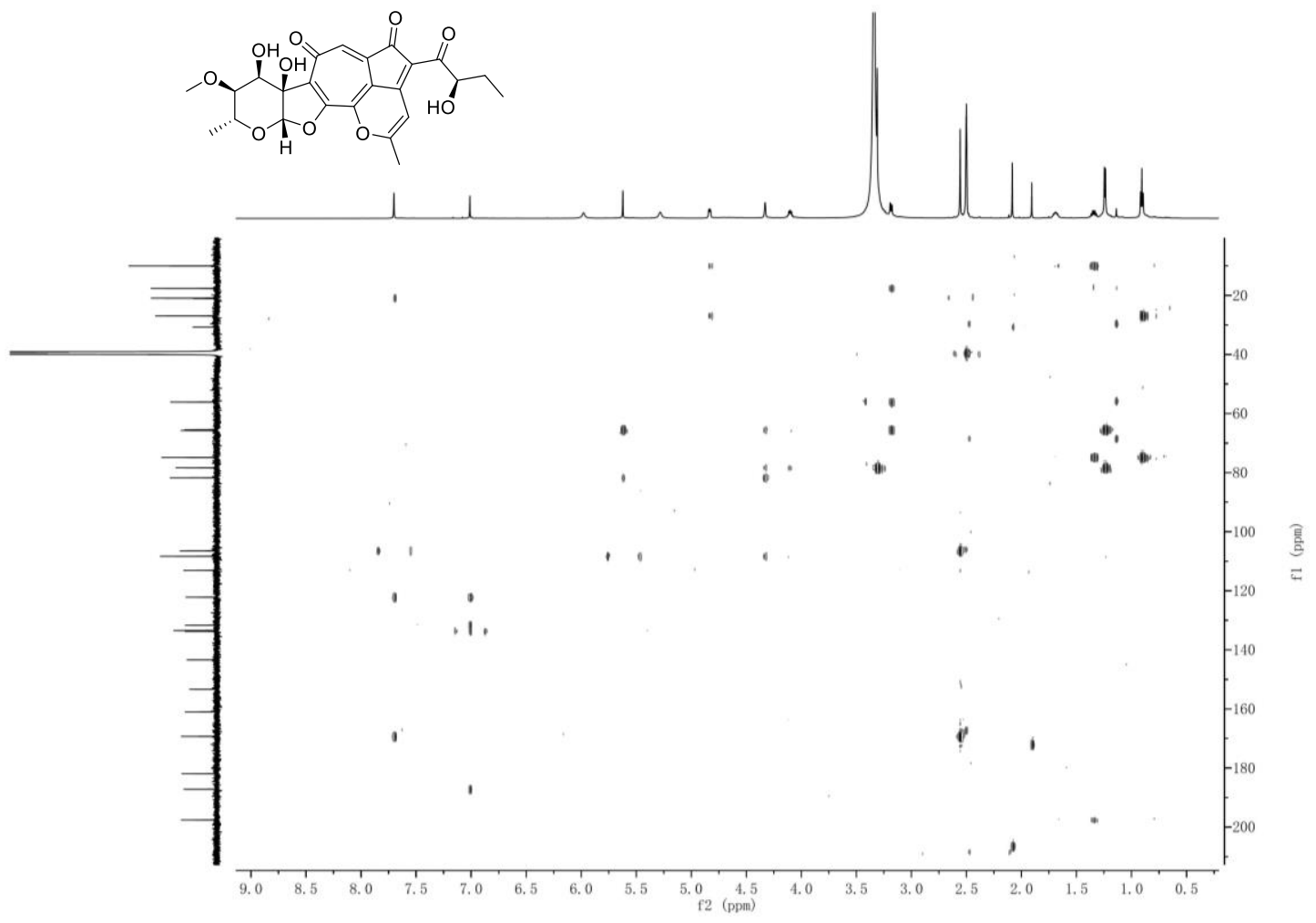

Figure S49. HMBC NMR spectrum of isatropolone C (6). 


\section{Relative energy calculation}

Single point energies in methanol solution using B3LYP/6-31G (d, p) method were calculated on the basis of the optimized structures in gas phase using the same method. Then, the total energies of each structure were calculated according to the Boltzmann statistics of each conformation. Results see Figure S50 and S52.

\section{ECD caculations.}

The theoretical calculations of isarubrolone C (1) and D (2) were carried out using Gaussian $09^{\mathrm{S} 1}$. Conformation search using molecular mechanics calculations was performed with MMFF force field with a $20 \mathrm{kcal} \mathrm{mol}^{-1}$ upper energy limit in MOE software (MOE2009.10) ${ }^{\mathrm{S} 2}$. The optimized conformation geometries and thermo dynamic parameters of all conformations were provided. The conformers were optimized at B3LYP/6-31G $(\mathrm{d}, \mathrm{p})$ level. The theoretical calculation of ECD was performed using time-dependent Density Functional Theory (TDDFT) at B3LYP/6-31+G (d, p) level in $\mathrm{MeOH}$ with PCM model. The ECD spectra of $\mathbf{1}$ and $\mathbf{2}$ were obtained by weighing the Boltzmann distribution rate of each geometric conformation.

The experimental spectrum of $\mathbf{1}$ was more consistent with the spectrum calculated for the 1Ba over the wavelength range examined. The absolute configuration of C-3 in $\mathbf{1}$ was therefore assigned as $R$. And keto-enol tautomerization was observed for $\mathbf{1}$ in methanol (Figure S50).

The experimental spectrum of $\mathbf{2}$ was more consistent with the spectrum calculated for the $\mathbf{2 a}$ over the wavelength range examined. And keto-enol tautomerization was observed for $\mathbf{2}$ in methanol (Figure S52).

\section{Reference:}

(S1) Gaussian 09, Revision C. 01. Gaussian, Inc., Wallingford CT, 2010.

(S2) MOE2009.10, Chemical Computing Group Inc., www.chemcomp.com.

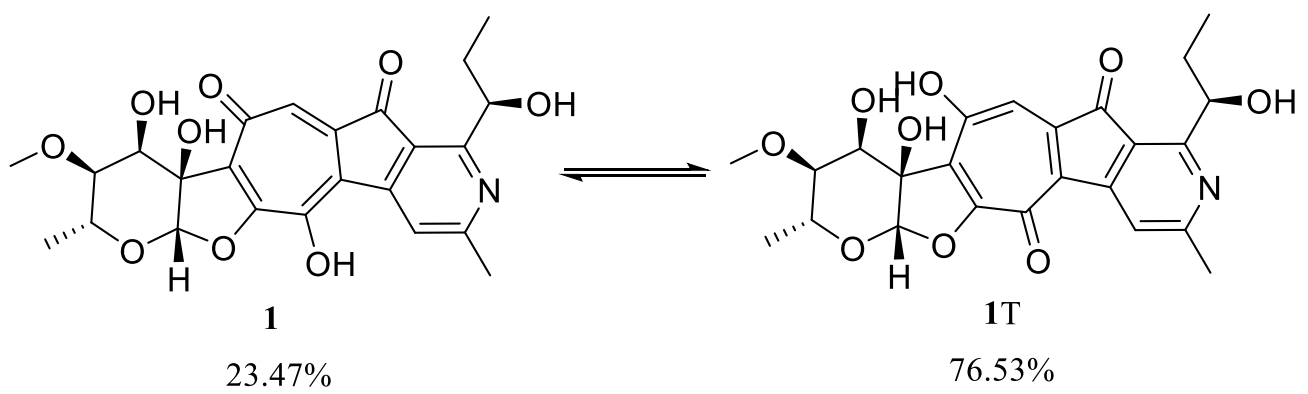

Figure S50. Keto-enol tautomerization for isarubrolone C (1). 
(a)
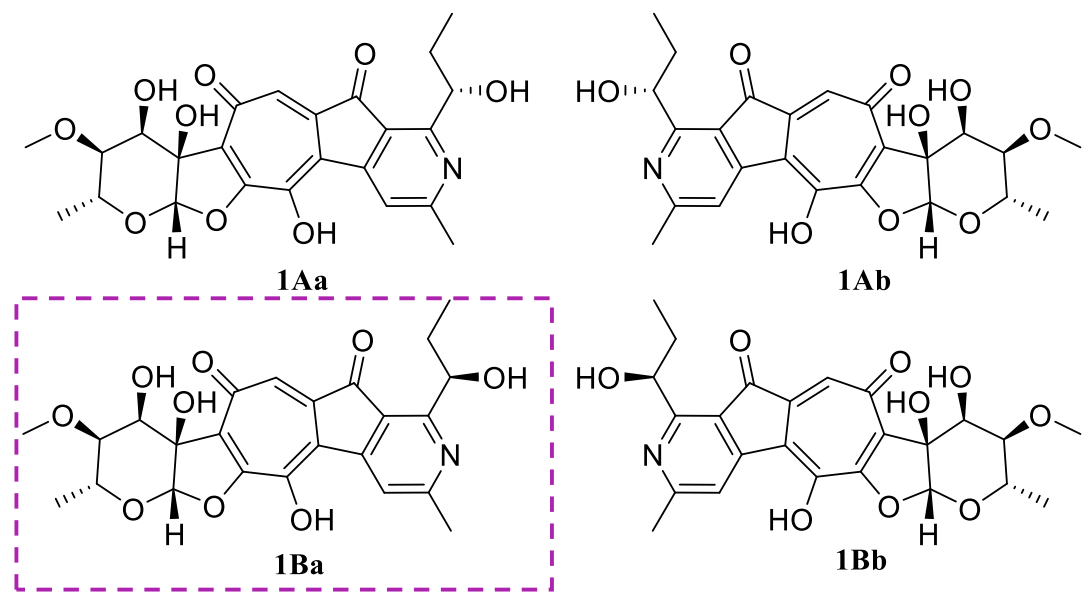

$1 \mathrm{Ab}$

(b)
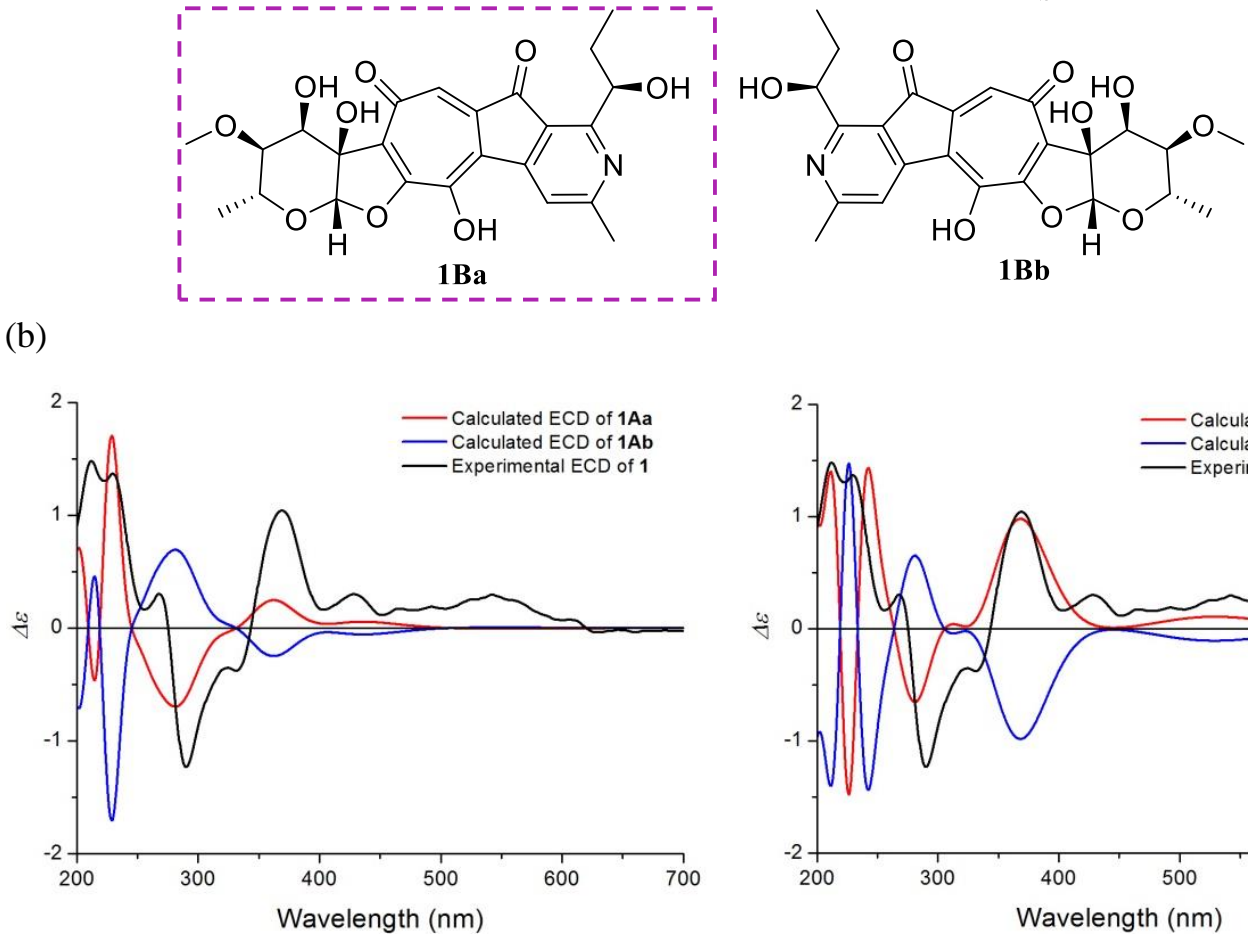

Figure S51. (a) Structures of compound 1 (1Ba) and it isomers (1Aa, 1Ab and 1Bb). (b) Experimental CD spectrum with the spectra calculated for its configuration.

The experimental spectrum of $\mathbf{1}$ was more consistent with the spectrum calculated for the $\mathbf{1 B a}$ over the wavelength range examined. The absolute configuration of $\mathrm{C}-3$ in $\mathbf{1}$ was therefore assigned as $R$. 
<smiles></smiles>

$97.32 \%$<smiles></smiles>

$2.68 \%$

Figure S52. Keto-enol tautomerization for isarubrolone D (2)

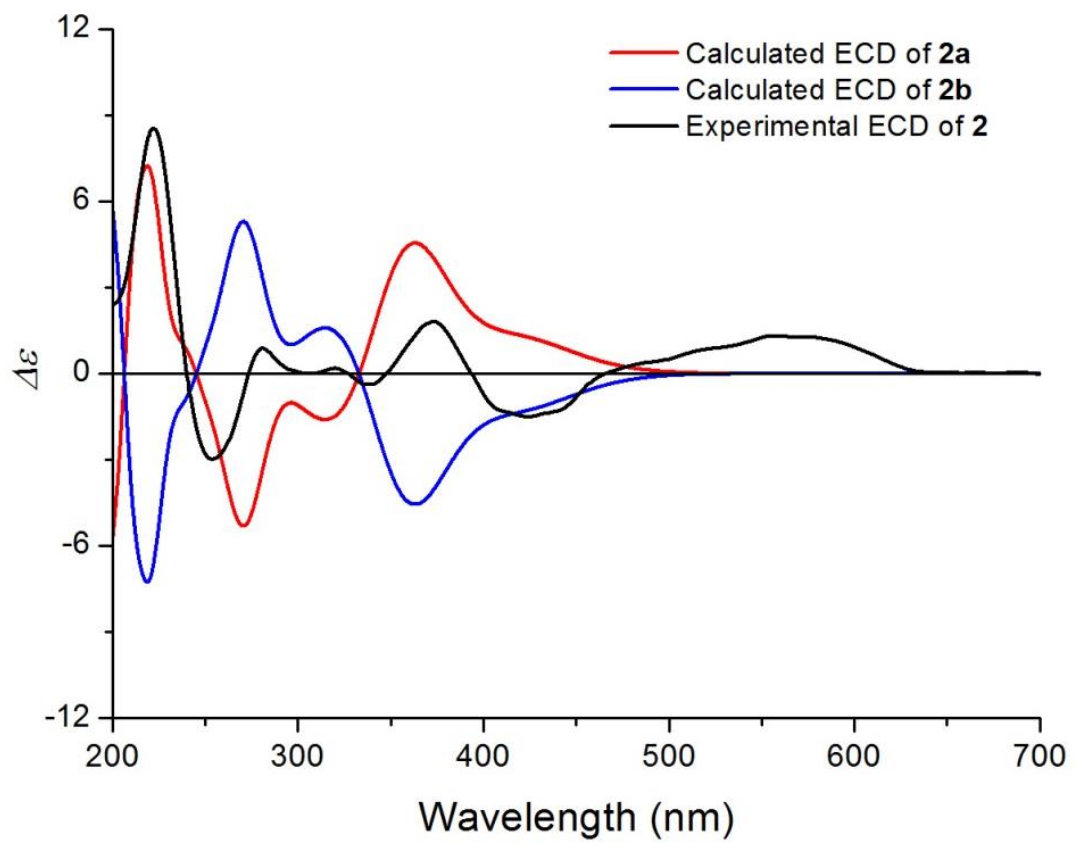

Figure S53. Assignment of the absolute configuration of isarubrolone D (2) by comparing its experimental CD spectrum with the spectra calculated for its configuration.

The experimental spectrum of $\mathbf{2}$ was more consistent with the spectrum calculated for the 2a over the wavelength range examined 


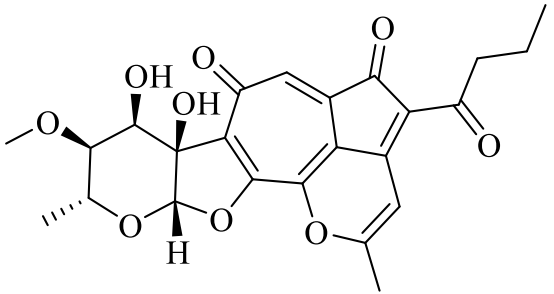

isatropolone A (5)

$$
||
$$

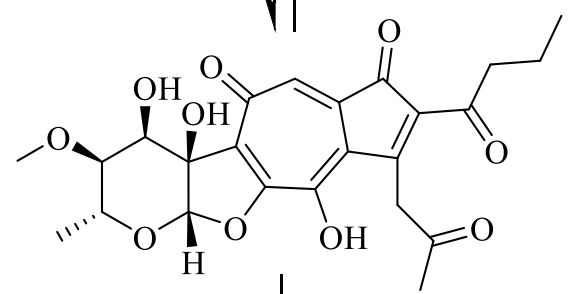<smiles>CCCc1c2c(cc(C)[n+]1-c1ccccc1C(=O)O)-c1c(O)cccc1C2=O</smiles>

$\mathrm{NH}_{3}$<smiles>CC(C)C</smiles>

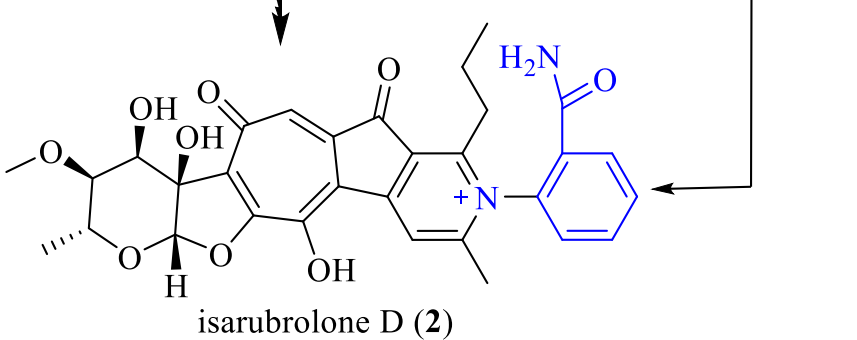<smiles>C=C(O[C@H]1C=C(C(=O)O)C=C[C@@H]1O)C(=O)O</smiles>

chorismate anthranilate
synthase $\begin{aligned} & \text { glutamine } \\ & \text { glutamate } \\ & \text { pyruvate }\end{aligned}$<smiles>Nc1ccccc1C(=O)O</smiles>

2-aminobenzoic acid

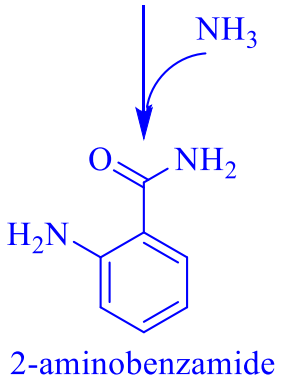

2-aminobenzamide

Figure S54. The plausible synthetic pathway for isarubrolone D (2) from isatropolone A (5) conjugated with aminobenzoic acid or aminobenzamide (derived from chorismate). 
In vitro chemical reaction of isatropolone $\mathbf{C}(6)$ with glycine, aminoacetone or $\mathbf{L}$-threonine A stock solution of 6 was prepared in THF $(10 \mathrm{mg} / \mathrm{mL})$. For each reaction $(100 \mu \mathrm{L}), 1 \mu \mathrm{L}$ of this solution was mixed in a $1.5 \mathrm{~mL}$ Eppendorf tube with $95 \mu \mathrm{L} \mathrm{H}_{2} \mathrm{O}$ and $4 \mu \mathrm{L}$ of $100 \mathrm{mM}$ stock solution glycine, aminoacetone or L-threonine. The reaction mixture was incubated at $37{ }^{\circ} \mathrm{C}$ with gentle shaking for 1 hour followed by LC-MS analysis.

LC traces

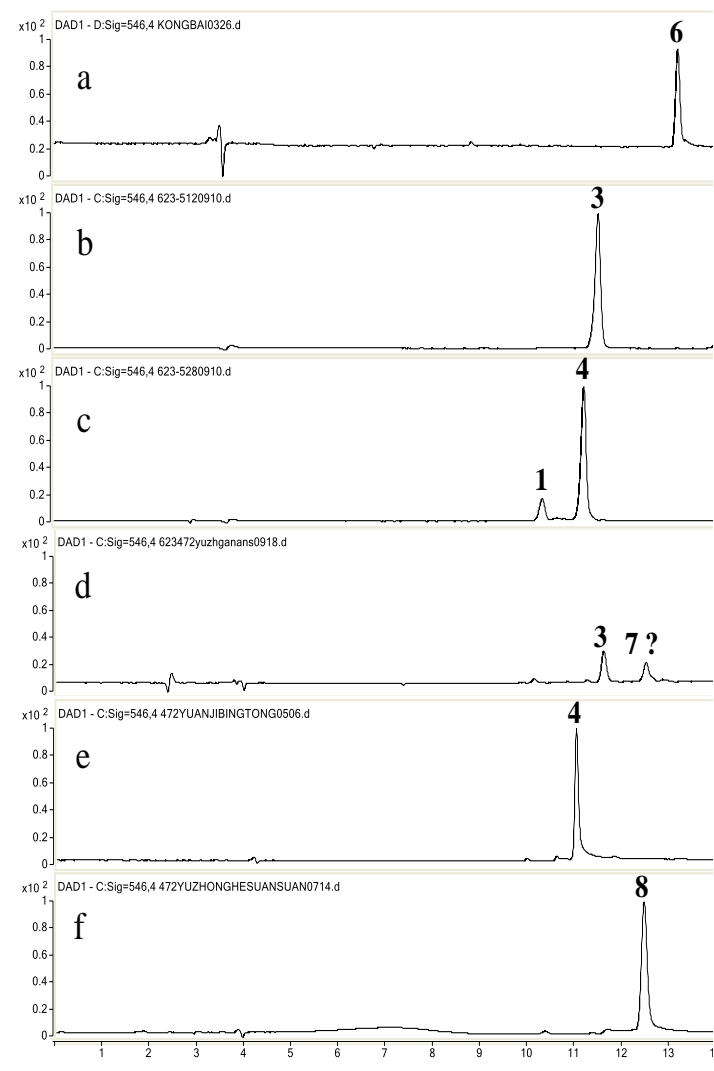

MS data

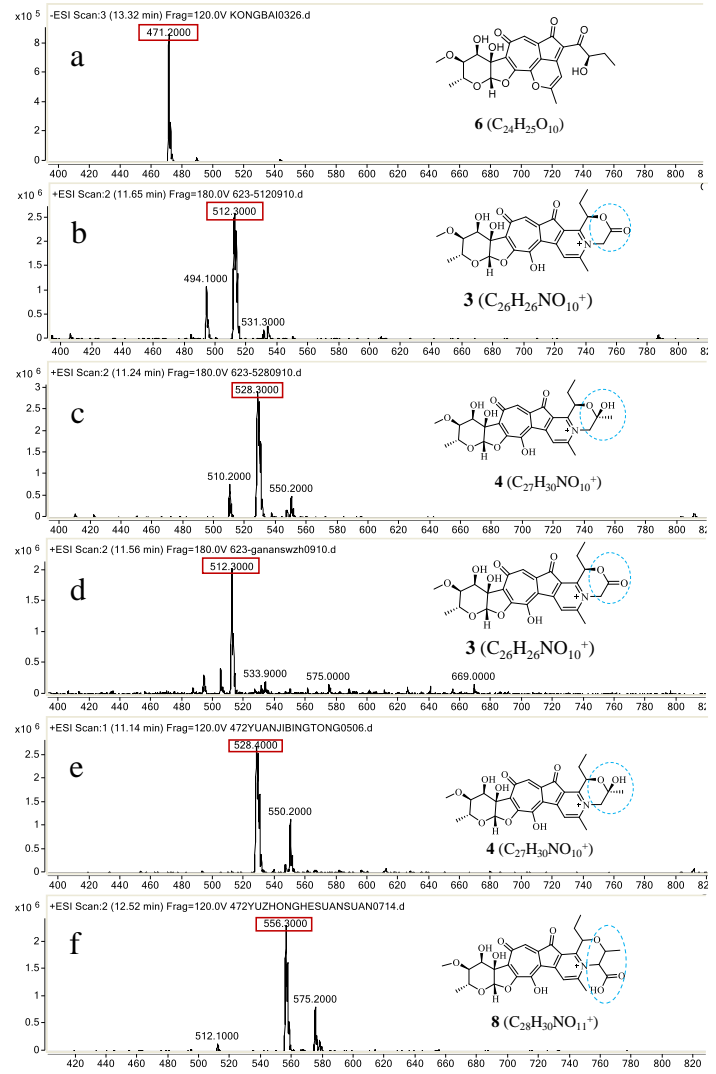

Figure 55. LC-ESIMS of in vitro chemical reaction product of isatropolone $C(\mathbf{6}, a ; \mathbf{3}, b ; 4, \mathbf{c})$ with glycine (d), aminoacetone (e) and threonine (f).

Compounds 3-4 were detected as expected, demonstrating that they were the conjugate of $\mathbf{6}$ with glycine or aminoacetone. New compounds 7-8 were also observed with deduced structures. 
Supplementing glycine in the medium increased the production of isarubrolone $E$ (3) by Streptomyces sp. CPCC 204095.

Fresh spores of Streptomyces sp. CPCC 204095 were spread on ISP2 plates (each contained $25 \mathrm{~mL}$ medium) supplemented with glycine at 1.0 and $2.0 \mathrm{~g} / \mathrm{L}(4.0 \mathrm{~g} / \mathrm{L}$ was found to inhibit the growth of Streptomyces sp. CPCC 204095), and then incubated for $2-7 \mathrm{~d}$ at $28{ }^{\circ} \mathrm{C}$. Each plate culture was extracted with $40 \mathrm{~mL}$ EtOAc for $2 \mathrm{~d}$. The extract was analyzed by HPLC to compare their isarubrolone $\mathrm{E}$ (3) content.

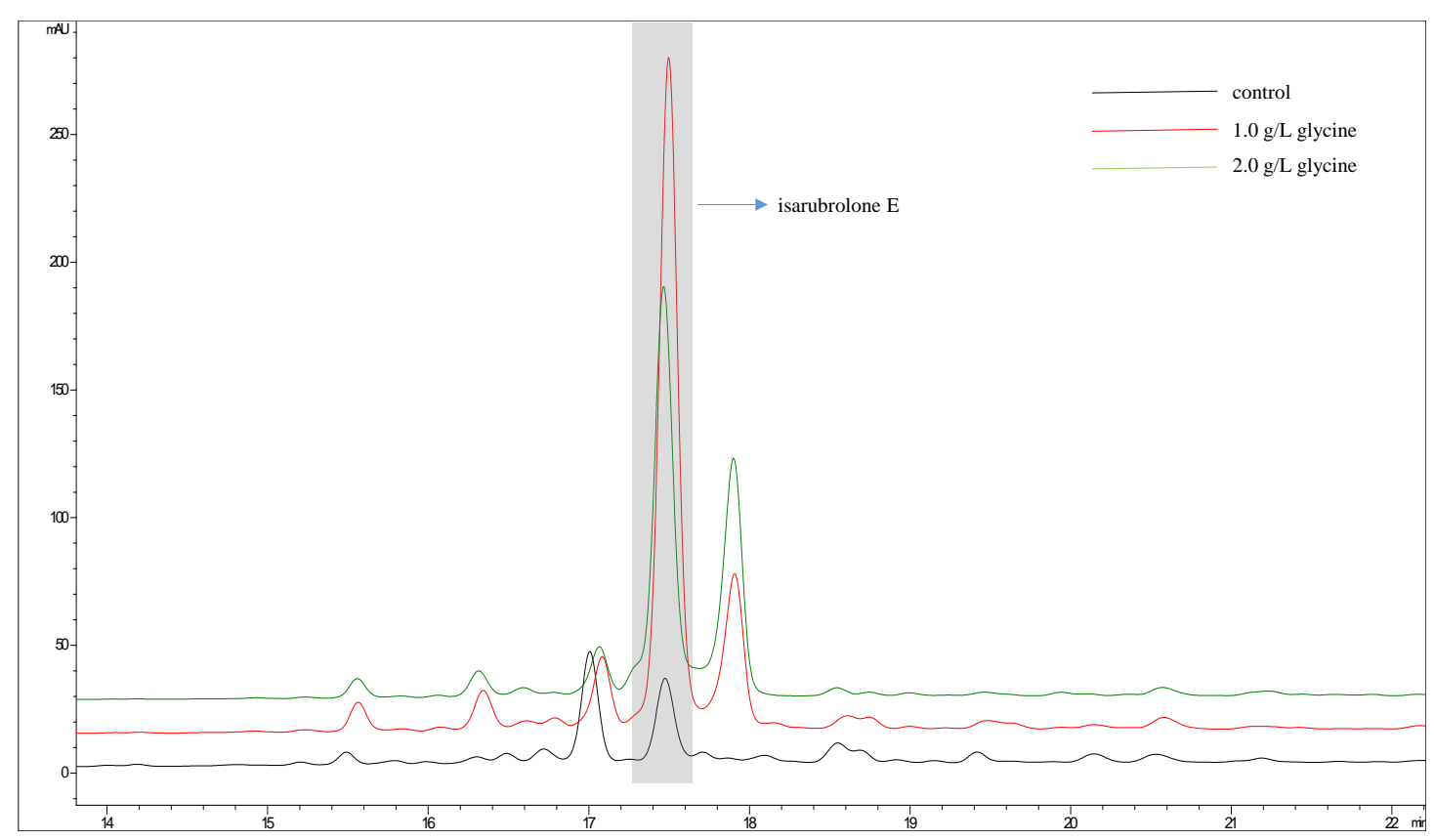

Figure 56. HPLC comparison of isarubrolone E (3) produced by Streptomyces sp. CPCC 204095 when glycine was supplemented into ISP2 medium. 


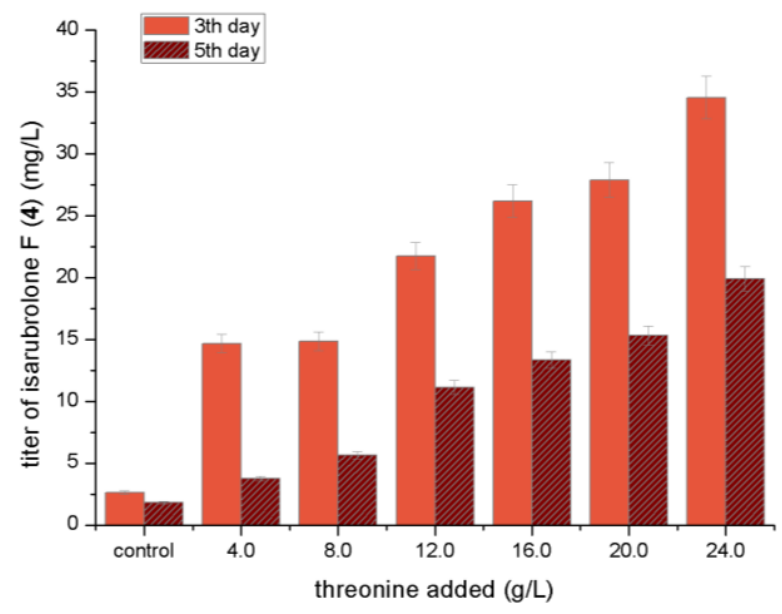

Figure S57. The titer of isarubrolone F (4) in relation to L-threonine supplemented in the culture medium for Streptomyces sp. CPCC 204095.

Fresh spores of Streptomyces sp. CPCC 204095 were spread on ISP2 medium plates (with each plate containing $25 \mathrm{ml}$ agar medium), and supplemented with L-threonine at 4.0, 8.0, 12.0, 16.0, 20.0 and $24.0 \mathrm{~g} / \mathrm{L}$, respectively. The plates were incubated at $28{ }^{\circ} \mathrm{C}$ for 3 or $5 \mathrm{~d}$. Each plate culture was then extracted with $40 \mathrm{~mL}$ EtOAc. The extract was concentrated and then analyzed by HPLC to compare the isarubrolone $\mathrm{F}(\mathbf{4})$ produced. The titers of isarubrolone $\mathrm{F}(\mathbf{4})$ was drawn in relation to L-threonine added in the medium.

In vitro chemical reaction of isatropolone $\mathrm{C}(6)$ with some amino acids and amines.

A stock solution of 6 was prepared in THF $(10 \mathrm{mg} / \mathrm{mL})$. For each reaction $(100 \mu \mathrm{L}), 1 \mu \mathrm{L}$ of this solution was mixed in a $1.5 \mathrm{~mL}$ Eppendorf tube with $95 \mu \mathrm{L} \mathrm{H} 2 \mathrm{O}$ and $4 \mu \mathrm{L}$ of $100 \mathrm{mM}$ stock solution of amino-2-propanol, L-lysine, L-histidine, 3-amino-2-methylbenzoic acid. The reaction mixture was incubated at $37{ }^{\circ} \mathrm{C}$ with gentle shaking for 1 hour followed by LC-MS analysis. 


\section{(1) LC traces}

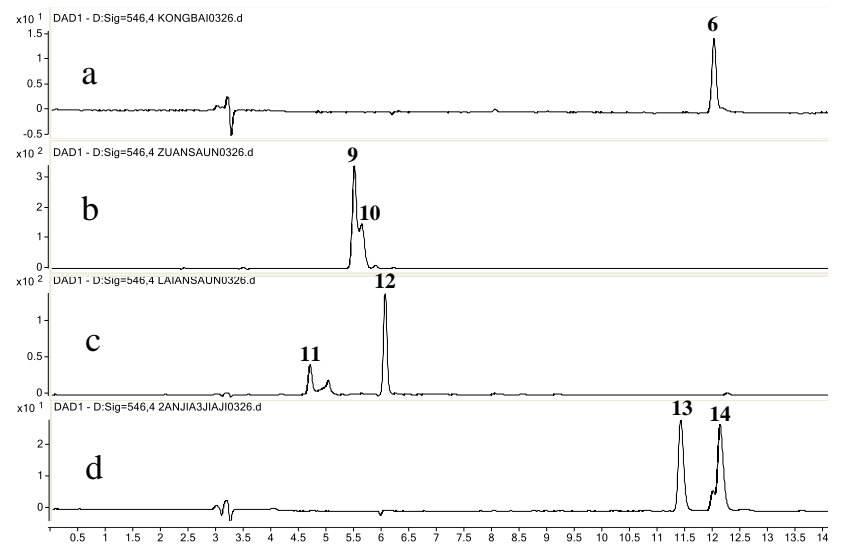

(2) MS data.
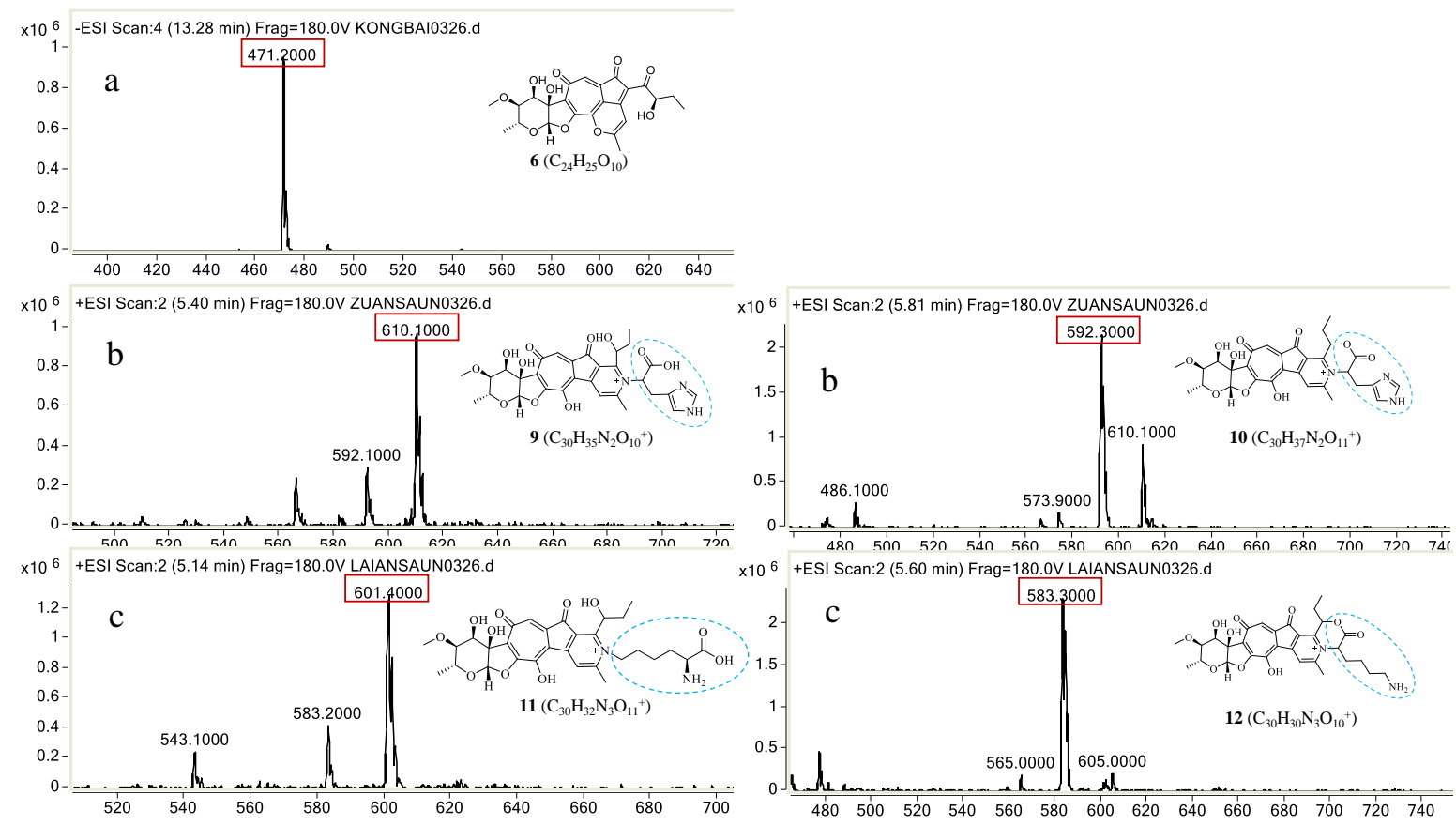

$\times 10^{6}+$ ESI Scan:2 (5.60 min) Frag=180.0V LAIANSAUN0326.
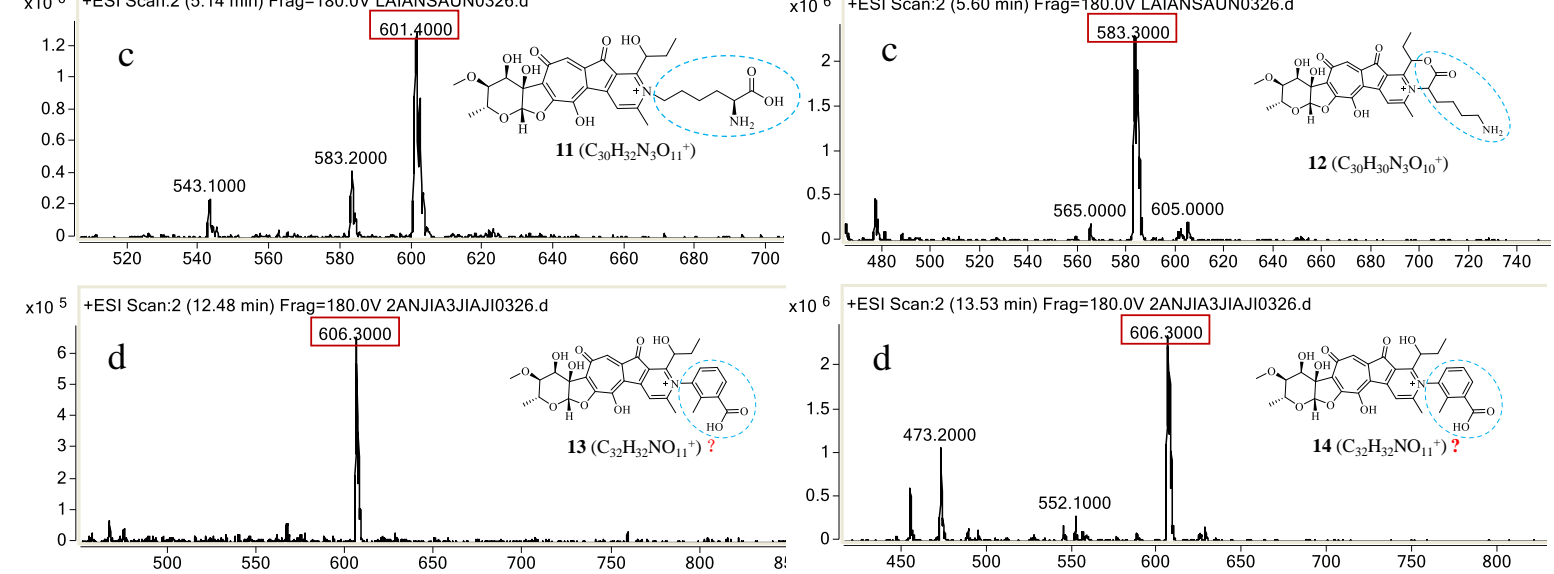

x10 ${ }^{6}+$ ESI Scan:2 (13.53 min) Frag=180.0V 2ANJIA3JIAJI0326.d

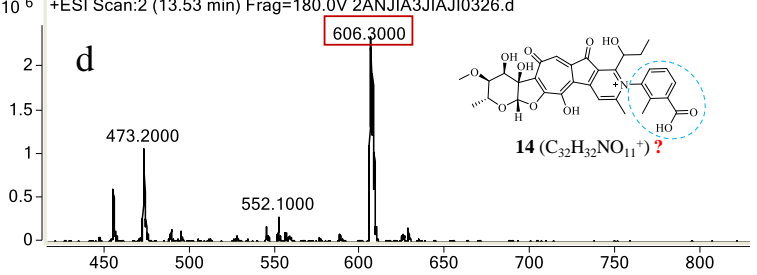

Figure S58. LC-ESIMS of in vitro chemical reaction product of isatropolone $\mathrm{C}(\mathbf{6}$, a) with histidine (b), lysine (c) and 3-amino-2-methylbenzoic acid (d).

New isarubrolones 9-14 were detected. 

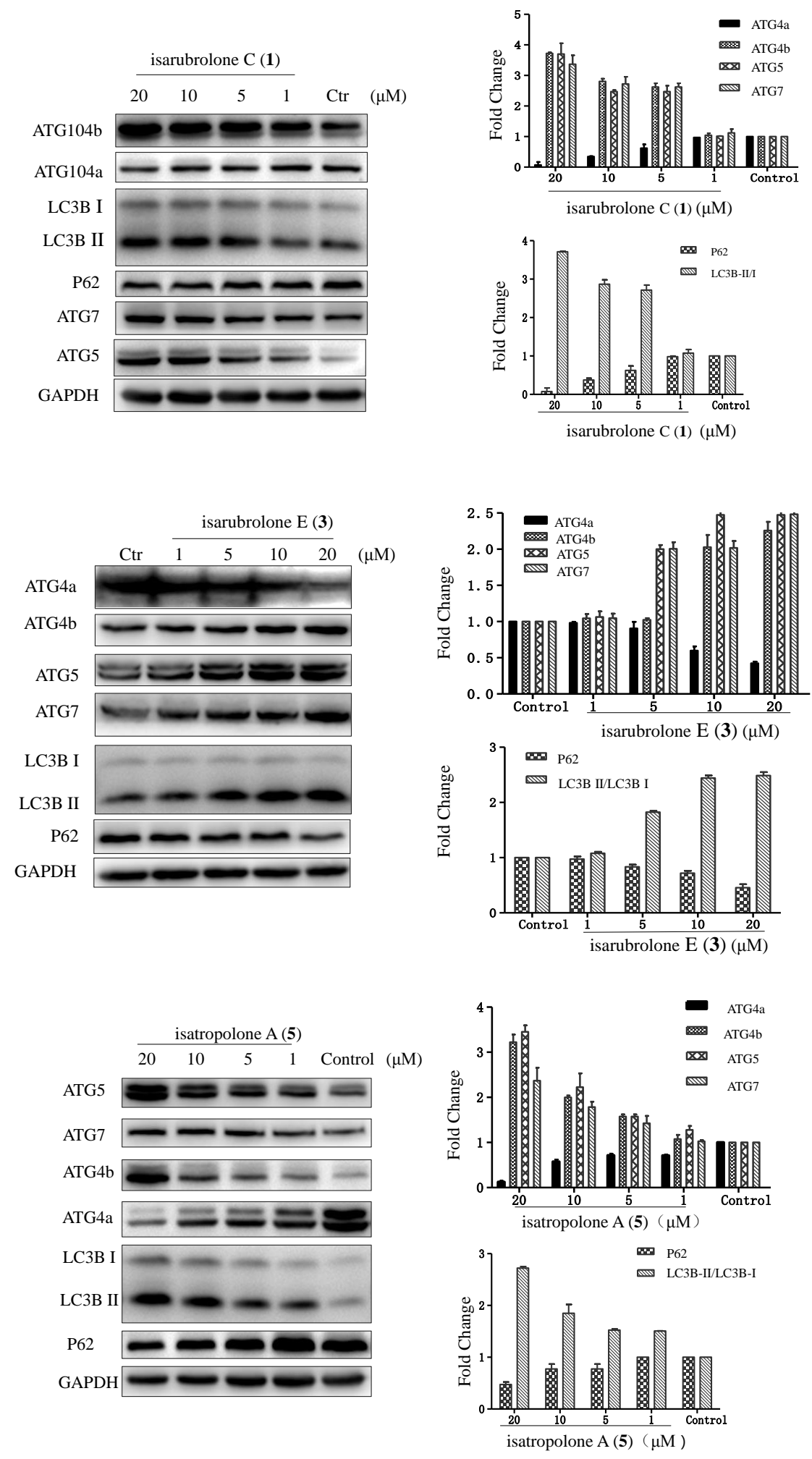

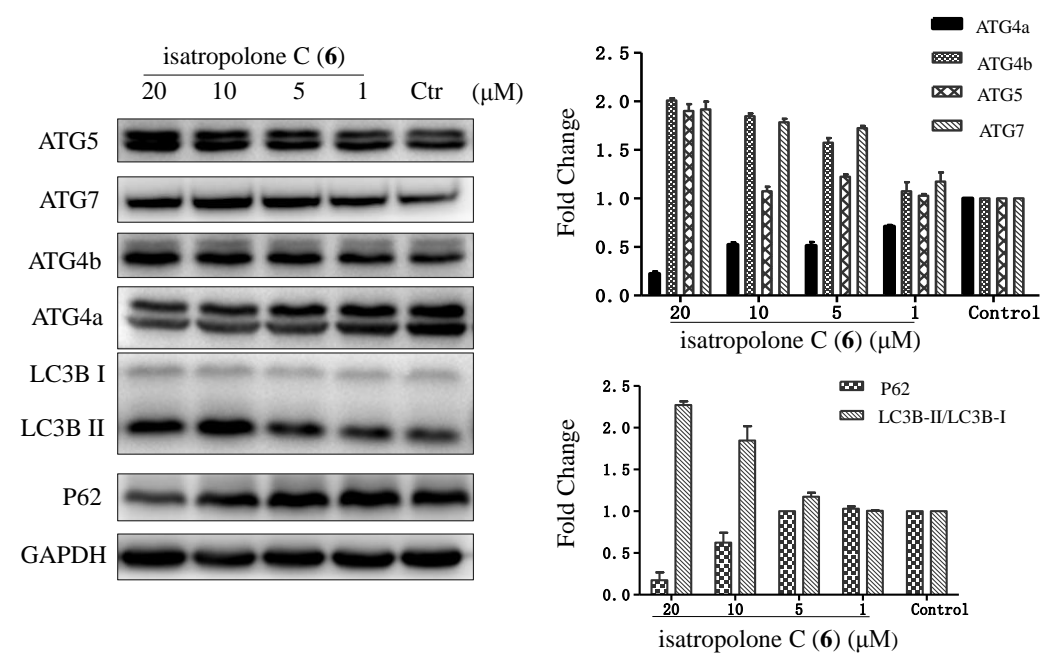

Figure S59. The autophagy inducing effect of isarubrolone C (1), isarubrolone E (3), isatropolone $\mathrm{A}(\mathbf{5})$ and isatropolone $\mathrm{C}(\mathbf{6})$. Western blotting showed the protein levels of autophagy proteins P62, LC3B-II/I, ATG4a/b, ATG7 and ATG5 in HepG2 cells.

HepG2 cells were exposed to isarubrolone C (1), isarubrolone E (3), isatropolone A (5) or isatropolone $\mathrm{C}(\mathbf{6})$ for $24 \mathrm{~h}$ at final concentrations of $1,5,10$, and $20 \mu \mathrm{M}$.

GADPH was used as a loading control.

\begin{abstract}
A brief description of autopahgic activity assay:
HepG2 cells were cultured in Minimum Essential Media (MEM) (Gibco) containing 10\% fetal bovine serum (FBS) (Gibco) with penicillin $(100 \mu \mathrm{g} / \mathrm{ml})$ and streptomycin $(100 \mu \mathrm{g} / \mathrm{ml})$. They were treated with isarubrolone or isatropolone for $24 \mathrm{~h}$ at final concentrations of $1,5,10$, or 20 $\mu \mathrm{M}$. The cells were then lysed in RIPA cell lysis buffer with protease inhibitor cocktail. Proteins were separated by $12 \%$ SDS-PAGE and transferred onto a nitrocellulose membrane. The membranes were incubated with anti-LC3B (M186-3, MBL), anti-P62 (PM045, MBL), anti-ATG5 (NB110-53818, Nuvus), anti-ATG7 (8558S, Cell Signaling Technology), anti-ATG4a (7613S, Cell Signaling Technology) and anti-ATG4b antibodies (13507S, Cell Signaling Technology) for quantification of autophagy marker proteins such as ATG4a/b, ATG5, ATG7, LC3B and P62.
\end{abstract}


Table S7. IC50 of isarubrolone C (1) and isarubrolone F (4) on tumor cells.

\begin{tabular}{ccccc}
\hline $\mathrm{IC}_{50}(\mu \mathrm{M})$ & $\mathrm{A} 549$ & HepG2 & HCT116 & H460 \\
\hline isarubrolone $\mathrm{C}$ & $916.70 \pm 115.41$ & $1486.82 \pm 349.94$ & $770.41 \pm 237.43$ & $1803.50 \pm 198.70$ \\
isarubrolone $\mathrm{F}$ & $505.61 \pm 34.18$ & $191.14 \pm 54.06$ & $440.82 \pm 80.47$ & $530.15 \pm 111.37$ \\
\hline
\end{tabular}

\section{A brief description of cytotoxicity assay}

Stock solutions of 1 and 4, dissolved in DMSO at $10.0 \mathrm{mg} / \mathrm{L}$ (21.1 and $18.9 \mathrm{mM}$, respectively), were used to evaluate their cytotoxicity against human lung cancer cell line (A549), human liver cancer cell line (HepG2), human colon cancer cell line (HCT116) and human large cell lung cancer (H460) by MTT assay. The assays were conducted at $0.5-250.0 \mu \mathrm{M}$ for $\mathbf{1}$ and $\mathbf{4}$. IC50 values were calculated for $\mathbf{1}$ and $\mathbf{4}$.

Reference: Sha, M.-Q.; Zhao, X.-L.; Li, L.; Li, L.-H.; Li, Y.; Dong, T.-G.; Niu, W.-X.; Jia, L.-J.; Shao, R.-G.; Zhen, Y.-S.; Wang, Z. Cell Death Dis. 2016, 7, 2486. 